hep-th/0612125

MAD-TH-06-12

\title{
Topological string theory on compact Calabi-Yau: modularity and boundary conditions
}

\author{
Min-xin Huang \&, Albrecht Klemm** and Seth Quackenbush \\ $\star \star \star \dagger$ Department of Physics, University of Wisconsin \\ ${ }^{\star}$ Department of Mathematics, University of Wisconsin \\ Madison, WI 53706, U.S.A.
}

\begin{abstract}
The topological string partition function $Z(\lambda, t, \bar{t})=\exp \left(\lambda^{2 g-2} F_{g}(t, \bar{t})\right)$ is calculated on a compact Calabi-Yau $M$. The $F_{g}(t, \bar{t})$ fulfill the holomorphic anomaly equations, which imply that $\Psi=Z$ transforms as a wave function on the symplectic space $H^{3}(M, \mathbb{Z})$. This defines it everywhere in the moduli space $\mathcal{M}(M)$ along with preferred local coordinates. Modular properties of the sections $F_{g}$ as well as local constraints from the 4 d effective action allow us to fix $Z$ to a large extent. Currently with a newly found gap condition at the conifold, regularity at the orbifold and the most naive bounds from Castelnuovo's theory, we can provide the boundary data, which specify $Z$, e.g. up to genus 51 for the quintic.
\end{abstract}

*minxin@physics.wisc.edu

**aklemm@physics.wisc.edu

†squackenbush@wisc.edu 


\section{Contents}

1 Outline 1

1.1 Extending the Seiberg-Witten approach to gravity . . . . . . . . 2

2 The topological B-model 4

2.1 The holomorphic anomaly equations ............. 4

2.2 Boundary conditions from light BPS states . . . . . . . . . . 8

3 Quintic 11

$3.1 \psi=\infty$ expansion and integer symplectic basis . . . . . . . . . . . 12

3.2 Polynomial expansion of $F^{(g)} \ldots \ldots \ldots \ldots \ldots$

3.3 Integration of the holomorphic anomaly equation . . . . . . . . . 15

3.4 Expansions around the orbifold point $\psi=0 \ldots \ldots . . . . . . .216$

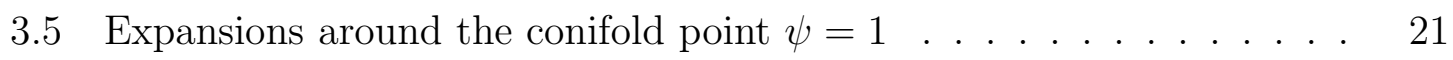

3.6 Fixing the holomorphic ambiguity: a summary of results . . . . . . 24

4 One-parameter Calabi-Yau spaces with three regular singular points 24

4.1 The integration of the anomaly equation ........... 25

4.2 The boundary behaviour . . . . . . . . . . . . . . 27

5 Symplectic invariants at large radius 32

5.1 Castelnuovo's theory and the cohomology of the BPS state moduli space 33

5.2 D-branes on the quintic . . . . . . . . . . . . . . 35

5.3 D-brane states on hypersurfaces in weighted projective space . . . . 38

5.4 D-branes on complete intersections . . . . . . . . . . . . 38

6 Conclusions 39

A Appendices

A.1 Classical intersection calculations using the adjunction formula . . . 42

A.2 Tables of Gopakumar-Vafa invariants . . . . . . . . . . . . . 43

A.3 Invariance of the generators under a change of the basis . . . . . . . 49

A.4 Symplectic basis, vanishing cycles and massless particles . . . . . . 51

\section{Outline}

Coupling topological matter to topological gravity is a key problem in string theory. Conceptually most relevant is the topological matter sector of the critical string as it arises e.g. in Calabi-Yau compactifications. Topological string theory on non-compact Calabi-Yau manifolds such as $\mathcal{O}(-3) \rightarrow \mathbb{P}^{2}$ is essentially solved either by localization 
- 1] or large N-techniques [2] and has intriguing connections to Chern-Simons theory [3], open-closed string duality [4], matrix models [5], integrable hierarchies of non-critical string theory [6] and 2d Yang-Mills theory [7].

However, while local Calabi-Yau manifolds are suitable to study gauge theories and more exotic field theories in $4 \mathrm{~d}$ and specific couplings to gravity, none of the techniques above extends to compact Calabi-Yau spaces, which are relevant for important questions in $4 \mathrm{~d}$ quantum gravity concerning e.g. the properties of $4 \mathrm{~d}$ black holes [10] and the wave function in mini superspace [11].

Moreover, while the genus dependence is encoded in the Chern-Simons and matrix model approaches in a superior fashion by the $\frac{1}{N^{2}}$-expansion, the moduli dependence on the parameter $t$ is reconstructed locally and in a holomorphic limit, typically by sums over partitions. This yields an algorithm, which grows exponentially in the world-sheet degree or the space-time instanton number.

As the total $F_{g}(t, \bar{t})$ are modular invariant sections over the moduli space $\mathcal{M}(M)$, they must be generated by a ring of almost holomorphic modular forms. This solves the dependence on the moduli in the most effective way. In the following we will show that space-time modularity, the holomorphic anomaly equations of Bershadsky, Cecotti, Ooguri and Vafa, as well as boundary conditions at various boundary components of the moduli space, solve the theory very efficiently.

For compact (and non-compact) Calabi-Yau spaces mirror symmetry is proven at genus zero. The modular properties that we need are also established at genus zero. Moreover it has been argued recently that the holomorphic anomaly recursions follow from categorical mirror symmetry [8, 9]. To establish mirror symmetry at higher genus, one needs merely to prove that the same boundary data fix the $F_{g}(t, \bar{t})$ in the $A$ - and the $B$-model.

\subsection{Extending the Seiberg-Witten approach to gravity}

Seiberg-Witten reconstructed the non-perturbative $\mathrm{N}=2$ gauge gauge coupling from meromorphic section over $\mathcal{M}(M)$ using their modular properties and certain local data from the effective action at singular divisors of $\mathcal{M}(M)$. In [12] we reconsidered the problem of topological string on local Calabi-Yau from the modular point of view and found that the singular behaviour of the gravitational couplings is restrictive enough to reconstruct them globally. This can be viewed as the most straightforward extension of the Seiberg-Witten approach to gravitational couplings.

Note that the problem of instanton counting in these cases is solved either by geometric engineering, one of the techniques mentioned above, or more directly by the localisation techniques in the moduli space of gauge theory instantons by Nekrasov, Nakajima et. al. It is nevertheless instructive to outline the general idea in this simple setting 1 . We focused on the $\mathrm{N}=2 S U(2)$ Seiberg-Witten case, but the features

\footnotetext{
${ }^{1}$ Maybe the simplest example of the relation between modularity and the holomorphic anomaly
} 
hold for any local Calabi-Yau whose mirror is an elliptic curve with a meromorphic differential [15, 13] $]^{2}$ and are as follows

- The genus $g$ topological string partition functions are given by

$$
F^{(g)}(\tau, \bar{\tau})=\xi^{2 g-2} \sum_{k=0}^{3(g-1)} \hat{E}_{2}^{k}(\tau, \bar{\tau}) c_{k}^{(g)}(\tau) .
$$

Here $\hat{E}_{2}(\tau, \bar{\tau}):=E_{2}(\tau)+\frac{6 i}{\pi(\bar{\tau}-\tau)}$ is the modular invariant anholomorphic extension of the second Eisenstein series $E_{2}(\tau)$ and the holomorphic 'Yukawa coupling' $\xi:=C_{t t t}^{(0)}=\frac{\partial \tau}{\partial t}$ is an object of weight -3 under the modular $\Gamma \in$ $\Gamma_{0}=S L(2, \mathbb{Z})$. For example for pure $N=2 S U(2)$ gauge theory $\Gamma=\Gamma(2)$ [14]. Modular invariance implies then that $c_{k}^{(g)}(\tau)$ are modular forms of $\Gamma$ of weight $6(g-1)-2 k$.

- The simple anti-holomorphic dependence of (1.1) implies that the only part in $F^{(g)}(\tau, \bar{\tau})$ not fixed by the recursive anomaly equations is the weight $6(g-1)$ holomorphic forms $c_{0}^{(k)}(\tau)$, which are finitely generated as a weighted polynomial $c_{0}^{(g)}(\tau)=p_{6(g-2)}\left(k_{1}, \ldots, k_{m}\right)$ in the holomorphic generators $G_{k_{1}}, \ldots, G_{k_{m}}$ of forms of $\Gamma$.

- The finite data needed to fix the coefficients in $p_{6(g-2)}\left(k_{1}, \ldots, k_{m}\right)$ are provided in part by the specific leading behaviour of the $F^{(g)}$ at the conifold divisor

$$
F_{\text {conifold }}^{(g)}=\frac{(-1)^{g-1} B_{2 g}}{2 g(2 g-2) t_{D}^{2 g-2}}+\mathcal{O}\left(t_{D}^{0}\right),
$$

in special local coordinates $t_{D}$. The order of the leading term was established in [22], the coefficient of the leading term in [34], and the 'gap condition,' i.e. the vanishing of the following $2 g-3$ negative powers in $t_{D}$ in [12]. This property in particular carries over to the compact case and we can give indeed a string theoretic explanation of the finding in [12].

- Further conditions are provided by the regularity of the $F^{(g)}$ at orbifold points in $\mathcal{M}(M)$. These conditions unfortunately turn out to be somewhat weaker in the global case than in the local case.

Similar forms as (1.1) for the $F^{(g)}$ appear in the context of Hurwitz theory on elliptic curves [18], of mirror symmetry in $K 3$ fibre limits [35] and on rational complex surfaces [16, 17]. In the local cases, which have elliptic curves as mirror geometry, we found [12, 13], that the above conditions (over)determine the unknowns in

equations is provided by Hurwitz theory on elliptic curves [18.

${ }^{2}$ With fairly obvious generalizations for the cases where the mirror is a higher genus curve. In this case the traditional modular forms of subgroups $\Gamma$ of $\Gamma_{0}:=S L(2, \mathbb{Z})$ have to be replaced by Siegel modular forms of subgroups of $S p(2 g, \mathbb{Z})[15$. 
$p_{6(g-2)}\left(k_{1}, \ldots, k_{m}\right)$ and solve the theory. This holds also for the gauge theories with matter, which from geometric engineering point of view correspond to local CalabiYau manifolds with several (Kähler) moduli [13]. Using the precise anholomorphic dependence and restrictions from space-time modularity one can iterate the holomorphic anomaly equation with an algorithm which is exact in the moduli dependence and grows polynomially in complexity with the genus.

Here we extend this approach further to compact Calabi-Yau spaces and focus on the class of one Kähler moduli Calabi-Yau spaces $M$ such as the quintic. More precisely we treat the class of one modulus cases whose mirror $W$ has, parameterized by a suitable single cover variable, a Picard-Fuchs system with exactly three regular singular points: The point of maximal monodromy, a conifold point, and a point with rational branching. The latter can be simply a $\mathbb{Z}_{d}$ orbifold point. This e.g. is the case for the hypersurfaces where the string theory has an exact conformal field theory description at this point in terms of an orbifold of a tensor product of minimal $(2,2)$ SCFT field theories, the so called the Gepner-model. For some complete intersections there are massless BPS particles at the branch locus, which lead in addition to logarithmic singularities.

We find a natural family of coordinates in which the conifold expansion as well as the rational branched logarithmic singularities exhibit the gap condition (1.2). Despite the fact that the modular group, in this case a $\operatorname{subgroup~of~} \operatorname{Sp}\left(h^{3}, \mathbb{Z}\right)$, is poorly understood 3 , we will see that the essential feature carry over to the compact case. Modular properties, the "gap condition", together with regularity at the orbifold, the leading behaviour of the $F_{g}$ at large radius, and Castelenovo's Bound determine topological string on one modulus Calabi-Yau to a large extent.

\section{The topological B-model}

In this section we give a quick summary of the approach of [21, 22] to the topological B-model, focusing as fast as possible on the key problems that need to be overcome: namely the problem of integrating the anomaly equation efficiently and the problem of fixing the boundary conditions.

\subsection{The holomorphic anomaly equations}

The definition of $F^{(g)}$ is $F^{(g)}=\int_{\mathcal{M}_{g}} \mu_{g}$ with measure on $\mathcal{M}_{g}$

$$
\mu_{g}=\prod_{i=1}^{3 g-3} \mathrm{~d} m_{i} \mathrm{~d} \bar{m}_{\bar{\imath}}\left\langle\prod_{i, \bar{\imath}} \int_{\Sigma} G_{z z} \mu_{\bar{z}}^{(i) z} \mathrm{~d}^{2} z \int_{\Sigma} G_{\bar{z} \bar{z}} \mu_{z}^{(i) \bar{z}} \mathrm{~d}^{2} z\right\rangle
$$

\footnotetext{
${ }^{3}$ Subgroups of $\operatorname{Sp}(4, \mathbb{Z})$ in which the monodromy group of the one-parameter models live have been recently specified [50].
} 
Here the Beltrami differentials $\mu_{\bar{z}}^{(i) z} \mathrm{~d} \bar{z}$ span $H^{1}(\Sigma, T \Sigma)$, the tangent space to $\mathcal{M}_{g}$. The construction of the measure $\mu_{g}$ is strikingly similar to the one for the bosonic string, once the BRST partner of the energy-momentum tensor is identified with the superconformal current $G_{z z} \mathrm{~d} z$ and the ghost number with the $U(1)$ charge [24]. \langle\rangle is to be evaluated in the internal $(2,2)$ SCFT, but it is easy to see that it gets only contributions from the topological $(c, c)$ sector.

The holomorphic anomaly equation reads for $g=1$ [21]

$$
\bar{\partial}_{\bar{k}} \partial_{m} F^{(1)}=\frac{1}{2} \bar{C}_{\bar{k}}^{i j} C_{m i j}^{(0)}+\left(\frac{\chi}{24}-1\right) G_{\bar{k} m},
$$

where $\chi$ is the Euler number of the target space $M$, and for $g>1$ [22]

$$
\bar{\partial}_{\bar{k}} F^{(g)}=\frac{1}{2} \bar{C}_{\bar{k}}^{i j}\left(D_{i} D_{j} F^{(g-1)}+\sum_{r=1}^{g-1} D_{i} F^{(r)} D_{j} F^{(g-r)}\right) .
$$

The right hand side of the equations comes from the complex co-dimension one boundary of the moduli space of the worldsheet $\mathcal{M}_{g}$, which corresponds to pinching of handles. The key idea is that $\bar{\partial}_{\bar{k}} F^{(g)}=\int_{\mathcal{M}_{g}} \bar{\partial} \partial \lambda_{g}$, where $\bar{\partial} \partial$ are derivatives on $\mathcal{M}_{g}$ so that $\bar{\partial}_{\bar{k}} F^{(g)}=\int_{\partial \mathcal{M}_{g}} \lambda_{g}$. The contribution to the latter integral is from the co-dimension one boundary $\partial \mathcal{M}_{g}$.

The first equation (2.4) can be integrated using special geometry up to a holomorphic function [21, which is fixed by the consideration in Sect. 2.2.

The equations (2.5) are solved in BCOV using the fact that due to

$$
\bar{D}_{\bar{i}} \bar{C}_{\bar{\jmath} \bar{k} \bar{l}}=\bar{D}_{\bar{\jmath}} \bar{C}_{\bar{\imath} \bar{k} \bar{l}}
$$

one can integrate

$$
\bar{C}_{\bar{\jmath} \bar{k} \bar{l}}=e^{-2 K} \bar{D}_{\bar{i}} \bar{D}_{\bar{\jmath}} \bar{\partial}_{\bar{k}} S
$$

as

$$
S_{\bar{\imath}}=\bar{\partial}_{\bar{\imath}} S, \quad S_{\bar{\imath}}^{j}=\bar{\partial}_{\bar{\imath}} S^{j}, \quad \bar{C}_{\bar{k}}^{i j}=\partial_{\bar{k}} S^{i j}
$$

The idea is to write the right hand side of $(2.5)$ as a derivative w.r.t. $\bar{\partial}_{\bar{k}}$. In the first step one writes

$$
\begin{aligned}
\bar{\partial}_{\bar{k}} F^{(g)}= & \bar{\partial}_{\bar{k}}\left(\frac{1}{2} S^{i j}\left(D_{i} D_{j} F^{(g-1)}+\sum_{r=1}^{g-1} D_{i} F^{(r)} D_{j} F^{(g-r)}\right)\right) \\
& -\frac{1}{2} S^{i j} \bar{\partial}_{\bar{k}}\left(D_{i} D_{j} F^{(g-1)}+\sum_{r=1}^{g-1} D_{i} F^{(r)} D_{j} F^{(g-r)}\right) .
\end{aligned}
$$

With the commutator $R_{i \bar{k} j}^{l}=-\bar{\partial}_{\bar{k}} \Gamma_{i j}^{l}=\left[D_{i}, \partial_{\bar{k}}\right]_{j}^{l}=G_{i \bar{k}} \delta_{j}^{l}+G_{j \bar{k}} \delta_{i}^{l}-C_{i j m}^{(0)} \bar{C}_{\bar{k}}^{m l}$ the second term can be rewritten so that the $\bar{\partial}_{\bar{k}}$ derivative acts in all terms directly on 
$F^{(g)}$. Then using $(2.4,2.5)$ with $g^{\prime}<g$ one can iterate the procedure, which produces an equation of the form

$$
\bar{\partial}_{\bar{k}} F^{(g)}=\bar{\partial}_{\bar{k}} \Gamma^{(g)}\left(S^{i j}, S^{i}, S, C_{i_{1}, \ldots, i_{n}}^{(<g)}\right)
$$

where $\Gamma^{(g)}$ is a functional of $S^{i j}, S^{i}, S$ and $C_{i_{1}, \ldots, i_{n}}^{(<g)}$. This implies that

$$
F^{(g)}=\Gamma^{(g)}\left(S^{i j}, S^{i}, S, C_{i_{1}, \ldots, i_{n}}^{(<g)}\right)+c_{0}^{(g)}(t)
$$

is a solution. Here $c_{0}^{(g)}(t)$ is the holomorphic ambiguity, which is not fixed by the recursive procedure. It is holomorphic in $t$ as well as modular invariant. The major conceptual problem of topological string theory on compact Calabi-Yau is to find the boundary conditions which fix $c_{0}^{(g)}(t)$. Note that the problem is not well defined without the constraints from modular invariance. Using the generalization of the gap condition in Sect. 2.2, the behaviour of the orbifold singularities in Sect. 3.4 and Castelnuovo's bound in Sect. 5.2 we can achieve this goal to a large extent.

Properties of the $\Gamma^{(g)}\left(S^{i j}, S^{i}, S, C_{i_{1}, \ldots, i_{n}}^{(<g)}\right)$ are established using the auxiliary action

$$
Z=\int \mathrm{d} x \mathrm{~d} \phi \exp (Y+\tilde{W})
$$

where

$$
\begin{aligned}
\tilde{W}(\lambda, x, \phi, t, \bar{t}) & =\sum_{g=0}^{\infty} \sum_{m=0}^{\infty} \sum_{n=0}^{\infty} \frac{1}{m ! n !} \tilde{C}_{i_{1}, \ldots, i_{n}, \phi^{m}}^{(g)} x_{i_{1}} \ldots x_{i_{n}} \phi^{m} \\
& =\sum_{g=0}^{\infty} \sum_{n=0}^{\infty} \frac{\lambda^{2 g-2}}{n !} C_{i_{1}, \ldots, i_{n}}^{(g)} x_{i_{1}} \ldots x_{i_{n}}(1-\phi)^{2-2 g-n}+\left(\frac{\chi}{24}-1\right) \log \left(\frac{1}{1-\phi}\right),
\end{aligned}
$$

with $C_{i_{1}, \ldots, i_{n}}^{(g)}=D_{i_{1}} \ldots D_{i_{n}} F^{(g)}$ and the "kinetic term" is given by

$$
Y(\lambda, x, \phi ; t, \bar{t})=-\frac{1}{2 \lambda^{2}}\left(\Delta_{i j} x^{i} x^{j}+2 \Delta_{i \phi} x^{i} \phi+\Delta_{\phi \phi} \phi^{2}\right)+\frac{1}{2} \log \left(\frac{\operatorname{det} \Delta}{\lambda^{2}}\right) .
$$

In [22] it was shown that $\exp (\tilde{W})$ fulfills an equation

$$
\frac{\partial}{\partial \bar{t}_{i}} \exp (\tilde{W})=\left[\frac{\lambda^{2}}{2} \bar{C}_{\bar{\imath}}^{j k} \frac{\partial^{2}}{\partial x^{j} \partial x^{k}}-G_{\bar{\imath} j} x^{j} \frac{\partial}{\partial \phi}\right] \exp (\tilde{W})
$$

that is equivalent to the holomorphic anomaly equations, by checking the coefficients of the $\lambda$ powers, and $\exp (Y)$ fulfills

$$
\frac{\partial}{\partial \bar{t}_{\bar{\imath}}} \exp (Y)=\left[-\frac{\lambda^{2}}{2} \bar{C}_{\bar{\imath}}^{j k} \frac{\partial^{2}}{\partial x^{j} \partial x^{k}}-G_{\bar{\imath} j} x^{j} \frac{\partial}{\partial \phi}\right] \exp (Y)
$$


implying that $\Delta_{i j}, \Delta_{i \phi}$ and $\Delta_{\phi \phi}$ are the inverses to the propagators $K^{i j}=-S^{i j}$, $K^{i \phi}:=-S^{i}$ and $K^{\phi \phi}:=-2 S$. A saddle point expansion of $Z$ gives

$$
\log (Z)=\sum_{g=2}^{\infty} \lambda^{2 g-2}\left[F^{(g)}-\Gamma^{(g)}\left(S^{i j}, S^{i}, S, C_{i_{1}, \ldots, i_{n}}^{(<g)}\right)\right]
$$

where $\Gamma^{(g)}\left(S^{i j}, S^{i}, S, C_{i_{1}, \ldots, i_{n}}^{(<g)}\right)$ is simply the Feynman graph expansion of the action (2.12) with the vertices $\tilde{C}_{i_{1}, \ldots, i_{n}, \phi^{m}}^{(g)}$ and the propagators above. Moreover it can be easily shown that $\frac{\partial}{\partial_{\bar{t}_{i}}} Z=0$, which implies to all orders that $F^{(g)}$ can be written as (2.11). This establishes the reduction of the whole calculation to the determination of the holomorphic modular invariant sections $c_{0}^{(g)}(t) \in \mathcal{L}^{2 g-2}$. However it also reflects the major technical problem in the approach of BCOV, namely that the procedure to determine the recursive anholomorphic part grows exponentially with the genus. It has been observed in [33] that in concrete cases the terms appearing in the Feynman graph expansion are not functionally independent. This is a hint for finitely generated rings of anholomorphic modular forms over $\mathcal{M}(M)$. Using the modular constraints systematically in each integration step Yamaguchi and Yau developed a recursive procedure for the quintic whose complexity grows asymptotically only polynomially, see (3.53).

Since the B-model is $2 \mathrm{~d}$ gravity coupled to $2 \mathrm{~d}$ matter, let us compare the situation with pure $2 \mathrm{~d}$ gravity, where the objects of interest are correlation functions of $\tau_{d_{i}}=$ $\left(2 d_{i}+1\right) ! !_{c_{1}}\left(L_{i}\right)^{d_{i}}$ which are forms on $\overline{\mathcal{M}}_{g}$ constructed from the descendent fields

$$
F_{g}\left(t_{0}, t_{1}, \ldots\right)=\sum_{\left\{d_{i}\right\}}\left\langle\prod \tau_{d_{i}}\right\rangle_{g} \prod_{r>0} \frac{t_{r}^{n_{r}}}{n_{r} !} .
$$

Here $\left\{d_{i}\right\}$ are the set of all non-negative integers and $n_{r}:=\operatorname{Card}\left(i: d_{i}=r\right)$.

The linear second order differential equations (2.15) is the small phase space analog of the Virasoro constraints

$$
L_{n} Z=0, \quad n \geq-1
$$

on $Z=e^{F}$ with $F=\sum_{g=0}^{\infty} \lambda^{2 g-2} F_{g}$ the free energy of 2 d topological gravity 42 . Indeed the $L_{n}$ with $\left[L_{n}, L_{m}\right]=(n-m) L_{n+m}$ are second order linear differential operators in the $t_{i}$. The non-linear $K d V$ Hierarchy, which together with dilaton and string equation are equivalent to (2.19) [42], and correspond in the small phase space of the B-model to the holomorphic anomaly equations (2.42.5). In the $A$ model approaches to topological string on Calabi-Yau manifolds, such as relative GW-theory, localisation or attempts to solve the theory via massive $(2,2)$ models, the descendents are introduced according to the details of the geometrical construction and then "summed away".

The combinatorial cumbersome information in the descendent sums is replaced in the B-model by the contraints from the modular group, holomorphicity and boundary 
information from the effective $4 \mathrm{~d}$ action. As a consequence of this beautiful interplay between space-time and world-sheet properties one needs only the small phase space equations (2.4, 2.5, 2.15)).

This approach requires the ability to relate various local expansions of $F^{(g)}$ near the boundary of the moduli space. Sensible local expansions (of terms in the effective action) are in locally monodromy invariant coordinates. As explained in [15] these coordinates in various patches are related by symplectic transformations on the phase space $H^{3}(M)$. The latter extend as metaplectic transformations to the wave function $\psi=Z$ of the topological string on the Calabi-Yau [54], which defines the transformation on the $F^{(g)}$. It will be important for us that the real polarisation [48, 15, 49] defines an unique splitting (2.11/3.54) of local expansions of the $F^{(g)}$ in the anholomorphic modular part determined by the anomaly equations and the holomorphic modular part $c_{0}^{(g)}(t)$. Aspects of the wave functions properties and the various polarizations have been further discussed in [48, 15, 49].

\subsection{Boundary conditions from light BPS states}

Boundaries in the moduli space $\mathcal{M}(M)$ correspond to degenerations of the manifold $M$ and general properties of the effective action can be inferred from the physics of the lightest states. More precisely the light states relevant to the $F^{(g)}$ terms in the $N=2$ actions are the BPS states. Let us first discuss the boundary conditions for $F^{(1)}$ at the singular points in the moduli space.

- At the point of maximal unipotent monodromy in the mirror manifold $W$, the Kähler areas, four, and six volumes of the original manifold $M$ are all large. Therefore the lightest string states are the constant maps $\Sigma_{g} \rightarrow p t \in M$. For these Kaluza-Klein reduction, i.e. a zero mode analysis of the A-twisted nonlinear $\sigma$-model is sufficient to calculate the leading behavioun of $F^{(1)}$ as [21]

$$
F^{(1)}=\frac{t_{i}}{24} \int c_{2} \wedge J_{i}+\mathcal{O}\left(e^{2 \pi i t}\right) .
$$

Here $2 \pi i t_{i}=\frac{X^{i}}{X^{0}}$ are the canonical Kähler parameters, $c_{2}$ is the second Chern class, and $J_{i}$ is the basis for the Kähler cone dual to 2-cycles $C_{i}$ defining the $t_{i}:=\int_{C_{i}} \hat{J}=\int_{C_{i}} \sum_{i} t_{i} J_{i}$.

- At the conifold divisor in the moduli space $\mathcal{M}(W), W$ develops a nodal singularity, i.e., a collapsing cycle with $S^{3}$ topology. As discussed in sect. 3.5 this corresponds to the vanishing of the total volume of $M$. The leading behaviour at this point is universally [23]

$$
F^{(1)}=\frac{1}{12} \log \left(t_{D}\right)+O\left(t_{D}\right) .
$$

\footnotetext{
${ }^{4}$ The leading of $F^{(0)}$ at this point is similarly calculated and given in (3.37).
} 
This leading behaviour has been physically explained as the effect of integrating out a non-perturbative hypermultiplet, namely the extremal black hole of [43]. Its mass $\sim t_{D}$, see (2.26), goes to zero at the conifold and it couples to the $U(1)$ vector in the $N=2$ vectormultiplet, whose lowest component is the modulus $t_{D}$. The factor $\frac{1}{12}$ comes from the gravitational one-loop $\beta$-function, which describes the running of the $U(1)$ coupling [41]. A closely related situation is the one of a shrinking lense space $S / G$. As explained in [44 one gets in this case several BPS hyper multiplets as the bound states of wrapped D-branes, which modifies the factor $\frac{1}{12} \rightarrow \frac{|G|}{12}$ in the one loop $\beta$-function (2.21).

- The gravitational $\beta$-function argument extends also to non-perturbative spectra arising at more complicated singularities, e.g. with gauge symmetry enhancement and adjoint matter [45].

For the case of the one parameter families the above boundary information and the fact is sufficient to fix the holopmorphic ambiguity in $F^{(1)}$.

To learn from the effective action point of view about the higher genus boundary behaviour, let us recall that the $F^{(g)}$ as in $F(\lambda, t)=\sum_{g=1}^{\infty} \lambda^{2 g-2} F^{(g)}(t)$ give rise to the following term:

$$
S_{1-\text { loop }}^{N=2}=\int \mathrm{d}^{4} x R_{+}^{2} F(\lambda, t),
$$

where $R_{+}$is the self-dual part of the curvature and we identify $\lambda$ with $F_{+}$, the selfdual part of the graviphoton field strength. As explained in [37, 38, see [24] for a review, the term is computed by a one-loop integral in a constant graviphoton background, which depends only on the left $\left(S O(4)=S U(2)_{L} \otimes S U(2)_{R}\right)$ Lorentz quantum numbers of BPS particles $P$ in the loop. The calculation is very similar to the normal Schwinger-loop calculation. The latter computes the one-loop effective action in an $U(1)$ gauge theory, which comes from integrating out massive particles $P$ coupling to a constant background $U(1)$ gauge field. For a self-dual background field $F_{12}=F_{34}=F$ it leads to the following one-loop determinant evaluation:

$$
S_{1-l o o p}^{S}=\log \operatorname{det}\left(\nabla+m^{2}+2 e \sigma_{L} F\right)=\int_{\epsilon}^{\infty} \frac{\mathrm{d} s}{s} \frac{\operatorname{Tr}(-1)^{f} \exp \left(-s m^{2}\right) \exp \left(-2 s e \sigma_{L} F\right)}{4 \sin ^{2}(s e F / 2)} .
$$

Here the $(-1)^{f}$ takes care of the sign of the log of the determinant depending on whether $P$ is a boson or a fermion, and $\sigma_{L}$ is the Cartan element in the left Lorentz representation of $P$. To apply this calculation to the $N=2$ supergravity case one notes, that the graviphoton field couples to the mass, i.e., we have to identify $e=m$. The loop has two $R_{+}$insertions and an arbitrary, (for the closed string action even) number, of graviphoton insertions. It turns out 38] that the only supersymmetric BPS states with the Lorentz quantum numbers

$$
\left[\left(\frac{1}{2}, \mathbf{0}\right)+2(\mathbf{0}, \mathbf{0})\right] \otimes \mathcal{R}
$$


contribute to the loop. Here $\mathcal{R}$ is an arbitrary Lorentz representation of $S O(4)$. Moreover the two $R_{+}$insertions are absorbed by the first factor in the Lorentz representation (2.24), and the coupling of the particles in the loop to $F_{+}$insertions in the $N=2$ evaluation works exactly as in the non-supersymmetric Schwinger-loop calculation above for $P$ in the representation $\mathcal{R}$.

What are the microscopic BPS states that run in the loop? They are related to non-perturbative RR states, which are the only charged states in the Type II compactification. They come from branes wrapping cycles in the Calabi-Yau, and as BPS states their masses are proportional to their central charge (3.38). For example, in the large radius in the type IIA string on $M$, the mass is determined by integrals of complexified volume forms over even cycles. E.g., the mass of a 2 brane wrapping a holomorphic curve $\mathcal{C}_{\beta} \in H^{2}(M, \mathbb{Z})$ is given by

$$
m_{\beta}=\frac{1}{\lambda} \int_{\mathcal{C}_{\beta} \in H^{2}(M, \mathbb{Z})}(i J+B)=\frac{1}{\lambda} 2 \pi i t \cdot \beta=: \frac{1}{\lambda} t_{\beta} .
$$

We note that $H^{2}(M, \mathbb{Z})$ plays here the role of the charge lattice. In the type IIB picture the charge is given by integrals of the normalized holomorphic $(3,0)$-form $\Omega$. In particular the mass of the extremal black hole that vanishes at the conifold is given by

$$
m_{B H}=\frac{1}{\lambda \int_{A_{D}} \Omega} \int_{S^{3}} \Omega=: \frac{1}{\lambda} t_{D}
$$

where $A_{D}$ is a suitable non-vanishing cycle at the conifold. It follows from the discussion in previous paragraph that with the identification $e=m$ and after a rescaling $s \rightarrow s \lambda / e$ in (2.23), as well as absorbing $F$ into $\lambda$, one gets a result for (2.22)

$$
F(\lambda, t)=\int_{\epsilon}^{\infty} \frac{\mathrm{d} s}{s} \frac{\operatorname{Tr}(-1)^{f} \exp (-s t) \exp \left(-2 s \sigma_{L} \lambda\right)}{4 \sin ^{2}(s \lambda / 2)} .
$$

Here $t$ are the regularized masses, c.f. (2.25) 2.26) of the light particles $P$ that are integrated out, $f$ is their spins in $\mathcal{R}$, and $\sigma_{L}$ is the Cartan element in the representation $\mathcal{R}$.

At the large volume point one can related the relevant BPS states actually to bound states of $D 2$ with and infinite tower of $D 0$ branes with quantized momenta along the $M$-theory circle. Moreover the left spin content of the bound state can be related uniquely to the genus of $\mathcal{C}_{\beta}$. This beautiful story leads, as explained in [37, 38], after summing over the to momenta of the $D 0$ states to (5.105), which together with Castelnuovo's bound for smooth curves leads to very detailed and valuable boundary information as explained in Sec. 5. It is important to note that all states are massive so that there are no poles in $F^{(g)}$ for $g>1$. Hence the leading contribution is regular and can be extracted from the constant $\beta=0$ contribution in (5.105) as

$$
\lim _{t \rightarrow \infty} F_{\text {A-model }}^{(g)}=\frac{(-1)^{g-1} B_{2 g} B_{2 g-2}}{2 g(2 g-2)(2 g-2) !} \cdot \frac{\chi}{2} .
$$


The moduli space of constant maps factors into tow components: the moduli space of the world sheet curve $\Sigma_{g}$ and the location of the image point, i.e. $M$. The measures on the components are $\lambda^{3 g-3}$ and $c_{3}\left(T_{M}\right)$ respectively, explaining the above formula.

Let us turn to type IIB compactifications near the conifold. As it was checked with the $\beta$-function in [41] there is precisely one BPS hypermultiplet with the Lorentz representation of the first factor in (2.24) becoming massless at the conifold. In this case the Schwinger-Loop calculation (2.27) simply becomes

$$
F\left(\lambda, t_{D}\right)=\int_{\epsilon}^{\infty} \frac{\mathrm{d} s}{s} \frac{\exp \left(-s t_{D}\right)}{4 \sin ^{2}(s \lambda / 2)}+\mathcal{O}\left(t_{D}^{0}\right)=\sum_{g=2}^{\infty}\left(\frac{\lambda}{t_{D}}\right)^{2 g-2} \frac{(-1)^{g-1} B_{2 g}}{2 g(2 g-2)}+\mathcal{O}\left(t_{D}^{0}\right) .
$$

Since there are no other light particles, the above equation (2.29) encodes all singular terms in the effective action. There will be regular terms coming from other massive states. This is precisely the gap condition.

\section{Quintic}

We consider the familiar case of the quintic hypersurface in $\mathbb{P}^{4}$. The topological string amplitudes $F^{(g)}$ were computed up to genus 4 in [22, 33] using the holomorphic anomaly equation, and fixing the holomorphic ambiguity by various geometric data. It was also observed a long time ago [34] that the leading terms in $F^{(g)}$ around the conifold point are the same as $c=1$ strings at the self-dual radius, thus providing useful information for the holomorphic ambiguity. We want to explore whether we can find coordinates in which the $F^{(g)}$ on the compact Calabi-Yau exhibit the gap structure around the conifold point that was recently found for local Calabi-Yau geometries [12]. In order to do this, it is useful to rewrite the topological string amplitudes as polynomials [20]. We briefly review the formalism in [20].

The quintic manifold $M$ has one Kähler modulus $t$ and its mirror $W$ has one complex modulus $\psi$ and is given by the equation 5

$$
W=x_{1}^{5}+x_{2}^{5}+x_{3}^{5}+x_{4}^{5}+x_{5}^{5}-5 \psi^{\frac{1}{5}} x_{1} x_{2} x_{3} x_{4} x_{5}=0 .
$$

There is a relation between $t$ and $\psi$ known as the mirror map $t(\psi)$. The mirror map and the genus zero amplitude can be obtained using the Picard-Fuchs equation on $W$

$$
\left\{\left(\psi \partial_{\psi}\right)^{4}-\psi^{-1}\left(\psi \partial_{\psi}-\frac{1}{5}\right)\left(\psi \partial_{\psi}-\frac{2}{5}\right)\left(\psi \partial_{\psi}-\frac{3}{5}\right)\left(\psi \partial_{\psi}-\frac{4}{5}\right)\right\} \omega=0
$$

We can solve the equation as an asymptotic series around $\psi \rightarrow \infty$.

\footnotetext{
${ }^{5}$ Here for later convenience we use a slightly different notation from that of 20 . Their notation is simply related to ours by a change of variable $\psi^{5} \rightarrow \psi$.
} 


\section{$3.1 \psi=\infty$ expansion and integer symplectic basis}

Here we set the notation for the periods in the integer symplectic basis on $W$ and the relation of this basis to the D-brane charges on $M$. Eqs. (3.34,3.37) and following, apply to all one parameter models.

The point $\psi=\infty$ has maximal unipotent monodromy and corresponds to the large radius expansion of the mirror $M$ [19]. In the variable $z=\frac{1}{5^{5} \psi}$ and using the definitions

$$
\omega(z, \rho):=\sum_{n=0}^{\infty} \frac{\Gamma(5(n+\rho)+1)}{\Gamma^{5}(n+\rho+1)} z^{n+\rho} \quad D_{\rho}^{k} \omega:=\left.\frac{1}{(2 \pi i)^{k} k !} \frac{\partial^{k}}{\partial^{k} \rho} \omega\right|_{\rho=0}
$$

one can write the solutions 23$]$

$$
\begin{aligned}
& \omega_{0}=\omega(z, 0)=\sum_{n=0}^{\infty} \frac{(5 n) !}{(n !)^{5}\left(5^{5} \psi\right)^{n}} \\
& \omega_{1}=D_{\rho} \omega(z, 0)=\frac{1}{2 \pi i}\left(\omega_{0} \ln (z)+\sigma_{1}\right) \\
& \omega_{2}=\kappa D_{\rho}^{2} \omega(z, \rho)-c \omega_{0}=\frac{\kappa}{2 \cdot(2 \pi i)^{2}}\left(\omega_{0} \ln ^{2}(z)+2 \sigma_{1} \ln (z)+\sigma_{2}\right) \\
& \omega_{3}=\kappa D_{\rho}^{3} \omega(z, \rho)-c \omega_{1}+e \omega_{0}=\frac{\kappa}{6 \cdot(2 \pi i)^{3}}\left(\omega_{0} \ln ^{3}(z)+3 \sigma_{1} \ln ^{2}(z)+3 \sigma_{2} \ln (z)+\sigma_{3}\right)
\end{aligned}
$$

The constants $\kappa, c, e$ are topological intersection numbers, see below. The $\sigma_{k}$ are also determined by (3.31). To the first few orders, $\omega_{0}=1+120 z+113400 z^{2}+\mathcal{O}\left(z^{3}\right)$, $\sigma_{1}=770 z+810225 z^{2}+\mathcal{O}\left(z^{3}\right), \sigma_{2}=1150 z+\frac{4208175}{2} z^{2}+\mathcal{O}\left(z^{3}\right)$ and $\sigma_{3}=-6900 z-$ $\frac{9895125}{2} z^{2}+\mathcal{O}\left(z^{3}\right)$

The solutions (3.33) can be combined into the period vector $\vec{\Pi}$ with respect to an integer symplectic basis $6\left(A^{i}, B_{j}\right)$ of $H^{3}(W, \mathbb{Z})$ as follows [19]:

$$
\vec{\Pi}=\left(\begin{array}{c}
\int_{B_{1}} \Omega \\
\int_{B_{2}} \Omega \\
\int_{A^{1}} \Omega \\
\int_{A^{2}} \Omega
\end{array}\right)=\left(\begin{array}{c}
F_{0} \\
F_{1} \\
X_{0} \\
X_{1}
\end{array}\right)=\omega_{0}\left(\begin{array}{c}
2 \mathcal{F}^{(0)}-t \partial_{t} \mathcal{F}^{(0)} \\
\partial_{t} \mathcal{F}^{(0)} \\
1 \\
t
\end{array}\right)=\left(\begin{array}{c}
\omega_{3}+c \omega_{1}+e \omega_{0} \\
-\omega_{2}-a \omega_{1}+c \omega_{0} \\
\omega_{0} \\
\omega_{1}
\end{array}\right) .
$$

Physically, $t$ is the complexified area of a degree one curve and is related by the mirror map

$$
\begin{aligned}
2 \pi i t(\psi) & =\int_{\mathcal{C}}(i J+B)=\frac{\omega_{1}}{\omega_{0}}=-\log \left(5^{5} \psi\right)+\frac{154}{625 \psi}+\frac{28713}{390625 \psi^{2}}+. . \\
\frac{1}{z}=5^{5} \psi & =\frac{1}{q}+770+421375 q+274007500 q^{2}+\ldots
\end{aligned}
$$

to $\psi$. In (3.36), we inverted (3.35) with $q=e^{2 \pi i t}$. The prepotential is given by

$$
\mathcal{F}^{(0)}=-\frac{\kappa}{3 !} t^{3}-\frac{a}{2} t^{2}+c t+\frac{e}{2}+f_{\text {inst }}(q)
$$

\footnotetext{
${ }^{6}$ With $A^{i} \cap B_{j}=\delta_{j}^{i}$ and zero intersections otherwise.
} 
where the instanton expansion $f_{\text {inst }}(q)$ vanishes in the large radius $q \rightarrow 0$ limit. The constants in (3.34)3.37) can be related to the classical geometry of the mirror manifold [23, 19]. Denote by $J \in H_{2}(M, \mathbb{Z})$ the Kähler form which spans the onedimensional Kähler cone. Then $\kappa=\int_{M} J \wedge J \wedge J$ is the classical triple intersection number, $c=\frac{1}{24} \int_{M} c_{2}\left(T_{M}\right) \wedge J$ is proportional to the evaluation of the second Chern class of the tangent bundle $T_{M}$ against $J, e=\frac{\zeta(3)}{(2 \pi i)^{3}} \int_{M} c_{3}\left(T_{M}\right)$ is proportional to the Euler number, and $a$ is related to the topology of the divisor $D$ dual to $J . A^{1}$ is topologically a $T_{\mathbb{R}}^{3}$, while analysis at the conifold shows that the dual cycle $B_{1}$ has the topology of an $S^{3}$.

The identification of the central charge formula for compactified type II supergravity [25] with the K-theory charge of $D$-branes as objects $A \in K(M)$ [26, 27],

$$
\vec{Q} \cdot \vec{\Pi}=-\int_{M} e^{-\hat{J}} \operatorname{ch}(A) \sqrt{\operatorname{td}(M)}=Z(A),
$$

with $\hat{J}=t(i J+B)$ for the one-dimensional Kähler cone, checks the $D$-brane interpretation of (3.34) [28, 31, 32] in the $q \rightarrow 0$ limit on the classical terms $\kappa, a, c$, but

misses the $\frac{\chi \zeta(3)}{(2 \pi i)^{3}}$ term. Based on their scaling with the area parameter $t$ the periods $\left(F_{0}, F_{1}, X_{0}, X_{1}\right)$ are identified with the masses of $\left(D_{6}, D_{4}, D_{0}, D_{2}\right)$ branes. For smooth intersections and $D$ the restriction of the hyperplane class we can readily calculate $\kappa, a, c, \chi$ using the adjunction formula, see Appendix A.

\subsection{Polynomial expansion of $F^{(g)}$}

¿From the periods, or equivalently the prepotential $\mathcal{F}^{(0)}$, we can compute the Kähler potential $K:=-\log \left(i\left(\bar{X}^{\bar{a}} F_{a}-X^{a} \bar{F}_{\bar{a}}\right)\right)$ and metric $G_{\psi \bar{\psi}}:=\partial_{\psi} \bar{\partial}_{\bar{\psi}} K$ in the moduli space. The genus zero Gromov-Witten invariants are obtained by expanding $\mathcal{F}^{(0)}$ in large Kähler parameter in a power series in $q$, see Section 5 .

We use the notation of [20] and introduce the following symbols:

$$
\begin{aligned}
& A_{p}:=\frac{\left(\psi \partial_{\psi}\right)^{p} G_{\psi \bar{\psi}}}{G_{\psi \bar{\psi}}}, \quad B_{p}:=\frac{\left(\psi \partial_{\psi}\right)^{p} e^{-K}}{e^{-K}}, \quad(p=1,2,3, \cdots) \\
& C:=C_{\psi \psi \psi} \psi^{3}, \quad X:=\frac{1}{1-\psi}=:-\frac{1}{\delta}
\end{aligned}
$$

Here $C_{\psi \psi \psi} \sim \frac{\psi^{-2}}{1-\psi}$ is the three point Yukawa coupling, and $A:=A_{1}=-\psi \Gamma_{\psi \psi}^{\psi}$ and $B:=B_{1}=-\psi \partial_{\psi} K$ are the Christoffel and Kähler connections. In the holomorphic limit $\bar{\psi} \rightarrow \infty$, the holomorphic part of the Kähler potential and the metric go like

$$
e^{-K} \sim \omega_{0}, \quad G_{\psi \bar{\psi}} \sim \partial_{\psi} t
$$

so in the holomorphic limit, the generators $A_{p}$ and $B_{p}$ are

$$
A_{p}=\frac{\left(\psi \partial_{\psi}\right)^{p}\left(\partial_{\psi} t\right)}{\partial_{\psi} t}, \quad B_{p}=\frac{\left(\psi \partial_{\psi}\right)^{p} \omega_{0}}{\omega_{0}}
$$


It is straightforward to compute them in an asymptotic series using the Picard-Fuchs equation. There are also some derivative relations among the generators,

$$
\psi \partial_{\psi} A_{p}=A_{p+1}-A A_{p}, \quad \psi \partial_{\psi} B_{p}=B_{p+1}-B B_{p}, \quad \psi \partial_{\psi} X=X(X-1) .
$$

The topological amplitudes in the "Yukawa coupling frame" are denoted as

$$
P_{g}:=C^{g-1} F^{(g)}, \quad P_{g}^{(n)}=C^{g-1} \psi^{n} C_{\psi^{n}}^{(g)} .
$$

The A-model higher genus Gromov-Witten invariants are obtained in the holomorphic limit $\bar{t} \rightarrow \infty$ with a familiar factor of $\omega_{0}$ as

$$
F_{\text {A-model }}^{(g)}=\lim _{\bar{t} \rightarrow \infty} \omega_{0}^{2(g-1)}\left(\frac{1-\psi}{5 \psi}\right)^{g-1} P_{g} .
$$

The $P_{g}^{(n)}$ are defined for $g=0, n \geq 3, g=1, n \geq 1$, and $g=2, n \geq 0$. We have the initial data

$$
\begin{aligned}
& P_{g=0}^{(3)}=1 \\
& P_{g=1}^{(1)}=-\frac{31}{3} B+\frac{1}{12}(X-1)-\frac{1}{2} A+\frac{5}{3},
\end{aligned}
$$

and using the Christoffel and Kähler connections we have the following recursion relation in $n$,

$$
P_{g}^{(n+1)}=\psi \partial_{\psi} P_{g}^{(n)}-\left[n(A+1)+(2-2 g)\left(B-\frac{1}{2} X\right)\right] P_{g}^{(n)} .
$$

One can also use the Picard-Fuchs equation and the special geometry relation to derive the following relations among generators:

$$
\begin{aligned}
& B_{4}=2 X B_{3}-\frac{7}{5} X B_{2}+\frac{2}{5} X B-\frac{24}{625} X \\
& A_{2}=-4 B_{2}-2 A B-2 B+2 B^{2}-2 A+2 X B+X A+\frac{3}{5} X-1 .
\end{aligned}
$$

By taking derivatives w.r.t. $\psi$ one see that all higher $A_{p}(p \geq 2)$ and $B_{p}(p \geq 4)$ can be written as polynomials of $A, B, B_{2}, B_{3}, X$. It is convenient to change variables from $\left(A, B, B_{2}, B_{3}, X\right)$ to $\left(u, v_{1}, v_{2}, v_{3}, X\right)$ as

$$
\begin{aligned}
& B=u, \quad A=v_{1}-1-2 u, \quad B_{2}=v_{2}+u v_{1}, \\
& B_{3}=v_{3}-u v_{2}+u v_{1} X-\frac{2}{5} u X .
\end{aligned}
$$

Then the main result of [20] is the following proposition.

Proposition: Each $P_{g}(g \geq 2)$ is a degree $3 g-3$ inhomogeneous polynomial of $v_{1}$, $v_{2}, v_{3}, X$, where one assigns the degree $1,2,3,1$ for $v_{1}, v_{2}, v_{3}, X$, respectively. 
For example, at genus two the topological string amplitude is

$$
\begin{aligned}
P_{2}= & \frac{25}{144}-\frac{625}{288} v_{1}+\frac{25}{24} v_{1}^{2}-\frac{5}{24} v_{1}^{3}-\frac{625}{36} v_{2}+\frac{25}{6} v_{1} v_{2}+\frac{350}{9} v_{3}-\frac{5759}{3600} X \\
& -\frac{167}{720} v_{1} X+\frac{1}{6} v_{1}^{2} X-\frac{475}{12} v_{2} X+\frac{41}{3600} X^{2}-\frac{13}{288} v_{1} X^{2}+\frac{X^{3}}{240} .
\end{aligned}
$$

The expression for the $P_{g}, g=1, \ldots, 12$ for all models discussed in this paper can be obtained in a Mathematica readable form on [62], see "Pgfile.txt."

\subsection{Integration of the holomorphic anomaly equation}

The anti-holomorphic derivative $\partial_{\bar{\psi}} B_{p}$ of $p \geq 2$ can be related to $\partial_{\bar{\psi}} B$ [20]. Assuming $\partial_{\bar{\psi}} A$ and $\partial_{\bar{\psi}} B$ are independent, one obtains two relations for $P_{g}^{(n)}$ from the BCOV holomorphic anomaly equation as the following:

$$
\begin{aligned}
\frac{\partial P_{g}}{\partial u} & =0 \\
\left(\frac{\partial}{\partial v_{1}}-u \frac{\partial}{\partial v_{2}}-u(u+X) \frac{\partial}{\partial v_{3}}\right) P_{g} & =-\frac{1}{2}\left(P_{g-1}^{(2)}+\sum_{r=1}^{g-1} P_{r}^{(1)} P_{g-r}^{(1)}\right)
\end{aligned}
$$

The first equation (3.49) implies there is no $u$ dependence in $P_{g}$, as already taken into account in the main proposition of [20]. The second equation (3.50) provides a very efficient way to solve $P_{g}$ recursively up to a holomorphic ambiguity. To do this, one writes down an ansatz for $P_{g}$ as a degree $3 g-3$ inhomogeneous polynomial of $v_{1}, v_{2}, v_{3}, X$, plugs in equation (3.50), and uses the lower genus results $P_{r}^{(1)}, P_{r}^{(2)}$ $(r \leq g-1)$ to fix the coefficients of the polynomials. The number of terms in general inhomogeneous weighted polynomials in $\left(v_{1}, v_{2}, v_{3}, X\right)$ with weights $(1,2,3,1)$ is given by the generating function

$$
\frac{1}{(1-x)^{3}\left(1-x^{2}\right)\left(1-x^{3}\right)}=\sum_{n=0}^{\infty} p(n) x^{n} .
$$

It is easy to see from the equation (3.50) that the terms $v_{1}, \ldots v_{1}^{2 g-4}$ vanish, which implies that the number of terms in $P_{g}$ is

$$
n_{g}=p(3 g-3)-(2 g-4),
$$

e.g. $n_{g}=14,62,185,435,877,1590,2666,4211,6344$ for $g=1, \ldots, 10$. Comparing with $\sum_{n=1}^{\infty} \tilde{p}(n) x^{n}=\frac{1}{(1-x)^{5}}$ it follows in particular that asymptotically

$$
n_{g} \preceq(3 g-3)^{4} .
$$

Note that equation (3.50) determines the term $\hat{P}_{g}\left(v_{1}, v_{2}, v_{3}, X\right)$ in

$$
P_{g}=: \hat{P}_{g}\left(v_{1}, v_{2}, v_{3}, X\right)+f^{(g)}(X)
$$


completely. We can easily understand why the terms in the modular as well as holomorphic ambiguity $f^{(g)}(X)$ are not fixed, by noting that the equation (3.50) does not change when we add a term proportional to $X^{i}$ to $P_{g}$. This ambiguity must have the form [33, 20]

$$
f^{(g)}=\sum_{i=0}^{3 g-3} a_{i} X^{i} .
$$

The maximal power of $X$ is determined by (2.29). This follows from (3.42) and the universal behaviour of $t_{D} \sim \delta+\mathcal{O}\left(\delta^{2}\right)$ at the conifold (3.73). Note in particular from (??) that $\hat{P}_{g}$ is less singular at that point. The minimal power in (3.55) follows from (2.28) for $\psi \sim \infty$ and the leading behaviour of the solutions in Sect. 3.1.

We will try to fix these $3 g-2$ unknown constants $a_{i}(i=0,1,2 \cdots 3 g-3)$ by special structure of expansions of $F^{(g)}$ around the orbifold point $\psi \sim 0$ and the conifold point $\psi \sim 1$. Before proceeding to this, we note the constant term is fixed by the known leading coefficients in large complex structure modulus limit $\psi \sim \infty$ in [35, 38, 39].

The leading constant terms in the A-model expansion $\psi \sim \infty$ come from the constant map from the worldsheet to the Calabi-Yau (2.28). The large complex structure modulus behavior of $X$ is

$$
X \sim \frac{1}{\psi} \sim q=e^{2 \pi i t}
$$

So only the constant term $a_{0}$ in the holomorphic ambiguity contributes to leading term in A-model expansions (2.28), and is thus fixed. We still have $3 g-3$ coefficients $a_{i}(i=1,2, \cdots, 3 g-3)$ to be fixed.

\subsection{Expansions around the orbifold point $\psi=0$}

To analyze the $F^{(g)}$ in a new region of the moduli space we have to find the right choice of polarization. To do this we analytically continue the periods to determine the symplectic pairing in the new region and pick the new choice of conjugated varaibles.

The solutions of the Picard-Fuchs equation around the orbifold point $\psi \sim 0$ are four power series solutions with the indices $\frac{1}{5}, \frac{2}{5}, \frac{3}{5}, \frac{4}{5}$

$$
\begin{aligned}
\omega_{k}^{\text {orb }} & =\psi^{\frac{k}{5}} \sum_{n=0}^{\infty} \frac{\left(\left[\frac{k}{5}\right]_{n}\right)^{5}}{[k]_{5 n}}\left(5^{5} \psi\right)^{n} \\
& =-\frac{\Gamma(k)}{\Gamma^{5}\left(\frac{k}{5}\right)} \int_{\mathcal{C}_{0}} \frac{\mathrm{d} s}{e^{2 \pi i s}-1} \frac{\Gamma^{5}\left(s+\frac{k}{5}\right)}{\Gamma(5 s+k)}\left(5^{5} \psi\right)^{s+\frac{k}{5}}, \quad k=1, \ldots, 4 .
\end{aligned}
$$




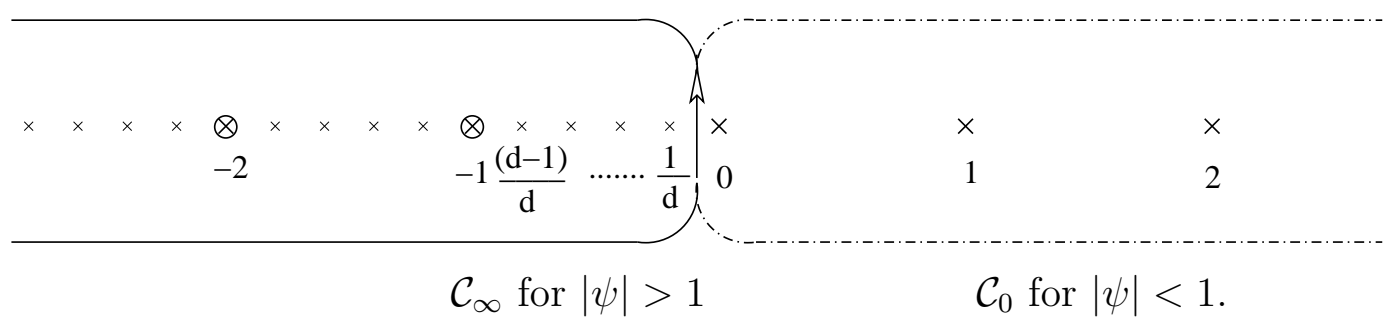

The Pochhammer symbol are defined as $[a]_{n}:=\frac{\Gamma(a+n)}{\Gamma(a)}$ and we normalized the first coefficient in $\omega_{k}^{\text {orb }}=\psi^{\frac{k}{5}}+\mathcal{O}\left(\psi^{\frac{5+k}{5}}\right)$ to one. The expression in the first line is recovered from the integral representation by noting that the only poles inside $\mathcal{C}_{0}$ for which the integral converges for $|\psi|<0$ are from $g(s)=\frac{1}{\exp (2 \pi i s)-1}$, which behaves at $s_{-n}^{\epsilon}=n-\epsilon, n \in \mathbb{N}$ as $g\left(s_{-n}^{\epsilon}\right) \sim-\frac{1}{2 \pi i \epsilon}$.

Up to normalization this basis of solutions is canonically distinguished, as it diagonalizes the $\mathbb{Z}_{5}$ monodromy at $\psi=0$. Similar as for the $\mathbb{C}^{3} / \mathbb{Z}_{3}$ orbifold [15], it can be viewed as a twist field basis. Here this basis is induced from the twist field basis of $\mathbb{C}^{5} / \mathbb{Z}_{5}$. As it was argued in [15] for $\mathbb{C}^{3} / \mathbb{Z}_{3}$, this twist field basis provides the natural coordinates in which the $F^{(g)}$ near the orbifold point can be interpreted as generating functions for orbifold Gromov-Witten invariants. Following up on foundational work on orbifold Gromov-Witten theory [51] and examples in two complex dimensions [52] this prediction has been checked by direct computation of orbifold Gromov-Witten invariants [53] at genus zero. This provides a beautiful check on the global picture of mirror symmetry.

As explained in [15] the relation between the large radius generating function of Gromov-Witten invariants and generating function of orbifold Gromov-Witten invariants is provided by the metaplectic transformation of the wave function [54. Since the change of the phase space variables from large radius to the orbifold the symplectic form is only invariant up to scaling one has to change the definition of the string coupling, which plays the role of $\hbar$ in the metaplectic transformation. These can be viewed as small phase space specialization of the metaplectic transformation on the large phase space [55].

Using the modular invariance of the anholomorphic $\hat{F}^{g}(t, \bar{t})$ it has been further shown in 15 that this procedure of obtaining the transformed holomorphic wave function is simply equivalent to taking the holomorphic limit on the $\hat{F}^{g}(t, \bar{t})$. This point was made [15] for the local case. But the only point one has to keep in mind for the global case is that $\hat{F}^{g}(t, \bar{t})$ are globally well defined sections of the Kähler line bundle. i.e. one has to perform a Kähler transformation along with the holomorphic limit.

We will now study the transformation from the basis (3.34) to the basis (3.57) to make B-model prediction along the lines of [15] with the additional Kähler transformation. Since the symplectic form $\omega$ on the moduli space is invariant under monodromy 
and $\omega_{k}^{\text {orb }}$ diagonalizes the $\mathbb{Z}_{5}$ monodromy, we must have in accordance with the expectation from the orbifold cohomology $H^{*}\left(\mathbb{C}^{5} / \mathbb{Z}_{5}\right)=\mathbb{C} \mathbf{1}_{0} \oplus \mathbb{C} \mathbf{1}_{1} \oplus \mathbb{C} \mathbf{1}_{2} \oplus \mathbb{C} \mathbf{1}_{3} \oplus \mathbb{C} \mathbf{1}_{4}$

$$
\omega=\mathrm{d} F_{k} \wedge \mathrm{d} X_{k}=-\frac{5^{4} s_{1}}{6} \mathrm{~d} \omega_{4}^{\text {orb }} \wedge \mathrm{d} \omega_{1}^{\text {orb }}+\frac{5^{4} s_{2}}{2} \mathrm{~d} \omega_{3}^{\text {orb }} \wedge \mathrm{d} \omega_{2}^{\text {orb }}
$$

The rational factors above have been chosen to match constraints from special geometric discussed below. Similarly the monodromy invariant Kähler potential must have the form

$$
e^{-K}=\sum_{k=1}^{4} r_{k} \omega_{k}^{\text {orb }} \overline{\omega_{k}^{\text {orb }}}
$$

To obtain the $s_{i}, r_{i}$ by analytic continuation to the basis (3.34) we follow [19] for the quintic and the generalisation in [46] for other cases and note that the integral converges for $|\psi|>1$ due to the asymptotics of the $f(s)=\frac{\Gamma^{5}(s+k / 5)}{\Gamma(5 s+k)}$ term, when the integral is closed along $\mathcal{C}_{\infty}$ [19]. At $s_{n}^{\epsilon}=-n-\epsilon$ the $g\left(s_{n}^{\epsilon}\right)$ pole is compensated by the $f\left(s_{n}^{\epsilon}\right)$ zero and at $s_{n, k}^{\epsilon}=-n-k / 5-\epsilon$ we note the expansions

$$
\begin{aligned}
g\left(s_{n, k}^{\epsilon}\right)= & \frac{\alpha^{k}}{1-\alpha^{k}}+\frac{2 \pi i \alpha^{k}}{\left(1-\alpha^{k}\right)^{2}} \epsilon+\frac{(2 \pi i)^{2} \alpha^{k}\left(1+\alpha^{k}\right)}{2\left(1-\alpha^{k}\right)^{3}} \epsilon^{2}+\frac{(2 \pi i)^{3} \alpha^{k}\left(1+4 \alpha^{k}+\alpha^{2 k}\right)}{6\left(1-\alpha^{k}\right)^{4}} \epsilon^{3}+\mathcal{O}\left(\epsilon^{4}\right) \\
f\left(s_{n, k}^{\epsilon}\right)= & \frac{\kappa \omega_{0}(n)}{\epsilon^{4}}+\frac{\kappa \sigma_{1}(n)}{\epsilon^{3}}+\frac{1}{\epsilon^{2}}\left(\frac{\kappa \sigma_{2}(n)}{2}+\frac{(2 \pi i)^{2} c_{2 J} \omega_{0}(n)}{24}\right)+ \\
& \frac{1}{\epsilon}\left(\frac{\kappa \sigma_{3}(n)}{6}+\frac{(2 \pi i)^{2} c_{2 J} \sigma_{1}(n)}{24}+\chi \zeta(3) \omega_{0}(n)\right)+\mathcal{O}\left(\epsilon^{0}\right) \\
\left(5^{5} \psi\right)^{s_{n}^{\epsilon}}= & z^{n}\left(1+\log (z) \epsilon+\frac{1}{2} \log (z)^{2} \epsilon^{2}+\frac{1}{6} \log (z)^{3} \epsilon^{3}+\mathcal{O}\left(\epsilon^{4}\right)\right)
\end{aligned}
$$

Here $\alpha=\exp (2 \pi i / 5) . \quad \kappa=\int_{M} J^{3}, c_{2 J}=\int_{M} c_{2} J$, and $\chi=\int_{M} c_{3}$ are calculated in App. A.17. The $\omega_{0}(n), \sigma_{i}(n)$ are coefficients of the series we encountered in sec. 3.1. Performing the residue integration and comparing with (3.33,3.34) we get

$$
\omega_{k}^{o r b}=\frac{(2 \pi i)^{4} \Gamma(k)}{\Gamma^{5}\left(\frac{k}{5}\right)}\left(\frac{\alpha^{k} F_{0}}{1-\alpha^{k}}-\frac{\alpha^{k} F_{1}}{\left(1-\alpha^{k}\right)^{2}}+\frac{5 \alpha^{k}\left(\alpha^{2 k}-\alpha^{k}+1\right) X_{0}}{\left(1-\alpha^{k}\right)^{4}}+\frac{\alpha^{k}\left(8 \alpha^{k}-3\right) X_{1}}{\left(1-\alpha^{k}\right)^{3}}\right)
$$

It follows with $r_{i}=\frac{\Gamma^{10}\left(\frac{k}{5}\right)}{\Gamma^{2}(k)} c_{i}$ that

$$
\begin{aligned}
c_{1} & =-c_{4}=\alpha^{2}(1-\alpha)\left(2+\alpha^{2}+\alpha^{3}\right), \quad c_{3}=-c_{2}=\alpha\left(2+\alpha-\alpha^{2}-2 \alpha^{3}\right) \\
s_{1} & =s_{2}=-\frac{1}{5^{5}(2 \pi i)^{3}} \\
\left(\begin{array}{c}
F_{0} \\
F_{1} \\
X_{0} \\
X_{1}
\end{array}\right) & =\psi^{1 / 5} \frac{\alpha \Gamma^{5}\left(\frac{1}{5}\right)}{(2 \pi i)^{4}}\left(\begin{array}{c}
(1-\alpha)\left(\alpha-1-\alpha^{2}\right) \\
\frac{1}{5}(8-3 \alpha)(1-\alpha)^{2} \\
\left(1-\alpha+\alpha^{2}\right) \\
\frac{1}{5}(1-\alpha)^{3}
\end{array}\right)+\mathcal{O}\left(\psi^{2 / 5}\right)
\end{aligned}
$$

\footnotetext{
${ }^{7}$ In fact using the generalization of (3.57) in [46] it is easily shown that the combinatorics, which leads to (A.116), are the same as the ones leading to the occurrence of the classical intersections here.
} 
Eq. (3.62) implies that up to a rational rescaling of the orbifold periods the transformation of the wave functions from infinity to the orbifold is given by a metaplectic transformation with the same rescaling of the string coupling as for the $\mathbb{C}^{3} / \mathbb{Z}_{3}$ case in [2]. Eq. (3.63) implies that there are no projective coordinates related to an $\operatorname{Sp}(4, \mathbb{Z})$ basis, which would vanish at the orbifold. This means that there is no massless RR state in the K-theory charge lattice which vanishes at the orbifold point. We note further that after rescaling of the orbifold periods the transformation (3.61) can be chosen to lie in $\operatorname{Sp}\left(4, \mathbb{Z}\left[\alpha, \frac{1}{5}\right]\right)$.

We can define the analogue of mirror map at the orbifold point,

$$
s=\frac{\omega_{2}^{\text {orb }}}{\omega_{1}^{\text {orb }}}=\psi^{\frac{1}{5}}\left(1+\frac{13 \psi}{360}+\frac{110069 \psi^{2}}{9979200}+\mathcal{O}\left(\psi^{3}\right)\right)
$$

where we use the notation $s$, as in [15], to avoid confusion with the mirror map in the large volume limit. We next calculate the genus zero prepotential at the orbifold point. For convenience let us rescale our periods $\hat{\omega}_{k-1}=5^{3 / 2} \omega_{k}^{\text {orb }}$. The Yukawa-Coupling is transformed to the $s$ variables as

$$
C_{s s s}=\frac{1}{\hat{\omega}_{0}^{2}} \frac{5}{\psi^{2}(1-\psi)}\left(\frac{\partial \psi}{\partial s}\right)^{3}=5+\frac{5}{3} s^{5}+\frac{5975}{6048} s^{10}+\frac{34521785}{54486432} s^{15}+\mathcal{O}\left(s^{20}\right) .
$$

A trivial consistency check of special geometry is that the genus zero prepotential $F^{(0)}=\int \mathrm{d} s \int \mathrm{d} s \int \mathrm{d} s C_{s s s}$ appears in the periods $\hat{\Pi}^{\text {orb }}=\left(\hat{\omega}_{0}, \hat{\omega}_{1}, \frac{5}{2 !} \hat{\omega}_{2},-\frac{5}{3 !} \hat{\omega}_{3}\right)^{T}$ as

$$
\hat{\Pi}^{o r b}=\hat{\omega}_{0}\left(\begin{array}{c}
1 \\
s \\
\partial_{s} F_{\mathrm{A}-\text { orbf. }}^{(0)} \\
2 F_{\mathrm{A}-\text { orbf. }}^{(0)}-s \partial_{s} F_{\mathrm{A}-\text { orbf. }}^{(0)}
\end{array}\right) .
$$

This can be viewed also as a simple check on the lowest order meta-plectic transformation of $\Psi$ which is just the Legendre transformation. Note that the Yukawa coupling is invariant under the $\mathbb{Z}_{5}$ which acts as $s \mapsto \alpha s$. $\mathbb{Z}_{5}$ implies further that there can be no integration constants, when passing from $C_{s s s}$ to $F_{0}$ and the coupling $\lambda$ must transform with $\lambda \mapsto \alpha^{\frac{3}{2}} \lambda$ to render $F(\lambda, s, \bar{s})$ invariant.

The holomorphic limit $\bar{\psi} \rightarrow 0$ of Kähler potential and metric follows from (3.59) by extracting the leading anti-holomorphic behaviour. Denoting 8 by $a_{k}$ the leading powers of $\omega_{k}^{o r b}$ we find

$$
\lim _{\bar{\psi} \rightarrow 0} e^{-K}=r_{1} \bar{\psi}^{a_{1}} \omega_{1}^{o r b}, \quad \lim _{\bar{\psi} \rightarrow 0} G_{\psi \bar{\psi}}=\bar{\psi}^{a_{2}-a_{1}-1} \frac{r_{2}}{r_{1}}\left(\frac{a_{2}}{a_{1}}-1\right) \frac{\partial s}{\partial \psi} .
$$

\footnotetext{
${ }^{8}$ This is to make contact with the other one modulus cases. Of course if $a_{1}=a_{2}$ a log singularity appears and the formula does not apply.
} 
Note that the constants and $\bar{\psi}$ and its leading power are irrelvant for the holomorphic limit of the generators (3.39)

$$
\begin{aligned}
X & =\frac{1}{1-\psi}=1+\psi+\psi^{2}+\mathcal{O}\left(\psi^{3}\right) \\
A & =-\frac{4}{5}+\frac{13}{60} \psi+\frac{3551}{18144} \psi^{2}+\mathcal{O}\left(\psi^{3}\right) \\
B & =\frac{1}{5}+\frac{1}{120} \psi+\frac{17}{4032} \psi^{2}+\mathcal{O}\left(\psi^{3}\right) \\
B_{2} & =\frac{1}{25}+\frac{7}{600} \psi+\frac{1027}{100800} \psi^{2}+\mathcal{O}\left(\psi^{3}\right) \\
B_{3} & =\frac{1}{125}+\frac{43}{3000} \psi+\frac{1633}{72000} \psi^{2}+\mathcal{O}\left(\psi^{3}\right)
\end{aligned}
$$

Using this information and integrating (3.44) we obtain the genus one free energy $F_{\mathrm{A} \text {-orbf. }}^{(1)}=-\frac{s^{5}}{9}+\ldots$ The regularity of $F^{(1)}$, i.e. the absence of $\log$ terms, is expected as there are no massless BPS states at the Gepner-point. Because of this, the considerations in Sec 2.2 imply also that the higher genus amplitudes

$$
F_{\text {A-orbf. }}^{(g)}=\lim _{\tilde{\tilde{t}} \rightarrow 0}\left(\hat{\omega}_{0}\right)^{2(g-1)}\left(\frac{1-\psi}{5 \psi}\right)^{g-1} P_{g}
$$

have no singularity at the orbifold point $\psi \sim 0$. This is in accordance with the calculations in [33] and implies that $\frac{P_{g}}{\psi^{\frac{3}{5}(g-1)}}$ is regular at $\psi \sim 0$.

The situation for the higher genus amplitudes for the compact $\mathcal{O}(5)$ constraint in $\mathbb{P}^{4}$ is considerably different from the one for the resolution $\mathcal{O}(-5) \rightarrow \mathbb{P}^{4}$ of the $\mathbb{C}^{5} / \mathbb{Z}_{5}$. In normal Gromov-Witten theory for genus $g>0$ on $\mathcal{O}(5)$ in $\mathbb{P}^{4}$ there is no bundle whose Euler class of its pullback from the ambient space $\mathbb{P}^{4}$ to the moduli space of maps gives rise to a suitable measure on $\mathcal{M}_{g, \beta}$ that counts the maps to the quintic. This is the same difficulties one has to face for higher genus calculation for the orbifold GW theory in $\mathcal{O}(5)$ in $\mathbb{P}^{4}$, and it is notably different 9 from the equivariant GW theory on $\mathbb{C}^{5} / \mathbb{Z}_{5}$.

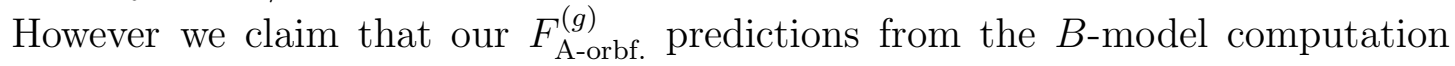
contain the information about the light even RR states at the orbifold point in useful variables and could in principle be checked in the A-model by some version of equivariant localisation. Below we give the first few order results. They are available to

\footnotetext{
${ }^{9}$ Since the brane bound state cohomology at infinity can only be understood upon including higher genus information, see Sec. 5 and 5.2, the claims that one can learn essential properties about the D-branes of the quintic at small volume from the $\mathbb{C}^{5} / \mathbb{Z}_{5}$ orbifold might be overly optimistic.
} 
genus 20 at 62 .

$$
\begin{array}{ll}
F_{\text {A-orbf. }}^{(0)} & =\frac{5 s^{3}}{6}+\frac{5 s^{8}}{1008}+\frac{5975 s^{13}}{10378368}+\frac{34521785 s^{18}}{266765571072}+\ldots \\
F_{\text {A-orbf. }}^{(1)} & =-\frac{s^{5}}{9}-\frac{163 s^{10}}{18144}-\frac{85031 s^{15}}{46702656}-\frac{6909032915 s^{20}}{20274183401472}+\ldots \\
F_{\text {A-orbf. }}^{(2)} & =\frac{155 s^{2}}{18}-\frac{5 s^{7}}{864}+\frac{585295 s^{12}}{14370048}+\frac{1710167735 s^{17}}{177843714048}+\ldots \\
F_{\text {A-orbf. }}^{(3)} & =\frac{488305 s^{4}}{9072}-\frac{3634345 s^{9}}{979776}-\frac{1612981445 s^{14}}{7846046208}-\frac{24262119333305 s^{19}}{116115777662976}+\ldots \\
F_{\text {A-orbf. }}^{(4)} & =\frac{48550 s}{567}+\frac{36705385 s^{6}}{163296}+\frac{16986429665 s^{11}}{603542016}+\frac{341329887875 s^{16}}{70614415872}+\ldots \\
F_{\text {A-orbf. }}^{(5)} & =\frac{1237460905 s^{3}}{224532}+\frac{108607458385 s^{8}}{28740096}-\frac{2079654832074515 s^{13}}{1553517149184}-\frac{50102421421803185 s^{18}}{438808843984896}+\ldots
\end{array}
$$

The holomorphic ambiguity (3.55) is a power series of $\psi$ starting from a constant term, so requiring $\frac{P_{g}}{\psi^{\frac{3}{5}(g-1)}}$ to be regular imposes

$$
\left\lceil\frac{3}{5}(g-1)\right\rceil
$$

number of relations in $a_{i}$ in (3.55), where $\left\lceil\frac{3}{5}(g-1)\right\rceil$ is the ceiling, i.e. the smallest integer greater or equal to $\frac{3}{5}(g-1)$. We note that the leading behaviour $\omega_{1}^{\text {orb }} \sim \psi^{\frac{1}{d}}$ with $d=5$ for the quintic, which is typical for an orbifold point in compact CalabiYau, and which "shields" the singularity and diminishes the boundary conditions at the orbifold point from $g-1$ to $\left\lceil\frac{d-2}{d}(g-1)\right\rceil$. If this period is non-vanishing at $\psi=0$ and it is indeed simply a constant for all local cases [13], one gets $g-1$ conditions, which together with the gap condition at the conifold and the constant map information is already sufficient to completely solve the model. An example of this type is $\mathcal{O}(-3) \rightarrow \mathbb{P}^{2}$.

\subsection{Expansions around the conifold point $\psi=1$}

An new feature of the conifold region is that there is an choice in picking the polarization, but as we will show the gap property is independent of this choice.

A basis of solutions of the Picard-Fuchs equation around the conifold point $\psi-1=$ $\delta \sim 0$ is the following

$$
\vec{\Pi}_{c}=\left(\begin{array}{c}
\omega_{0}^{c} \\
\omega_{1}^{c} \\
\omega_{2}^{c} \\
\omega_{3}^{c}
\end{array}\right)=\left(\begin{array}{l}
1+\frac{2 \delta^{3}}{625}-\frac{83 \delta^{4}}{18750}+\frac{757 \delta^{5}}{156250}+\mathcal{O}\left(\delta^{6}\right) \\
\delta-\frac{3 \delta^{2}}{10}+\frac{11 \delta^{3}}{25}-\frac{217 \delta^{4}}{2500}+\frac{889 \delta^{5}}{15625}+\mathcal{O}\left(\delta^{6}\right) \\
\delta^{2}-\frac{23 \delta^{3}}{30}+\frac{1049 \delta^{4}}{1800}-\frac{34343 \delta^{5}}{75000}+\mathcal{O}\left(\delta^{6}\right) \\
\omega_{1}^{c} \log (\delta)-\frac{9 d^{2}}{20}-\frac{169 d^{3}}{450}+\frac{27007 d^{4}}{90000}-\frac{152517 d^{5}}{625000}+\mathcal{O}\left(\delta^{6}\right)
\end{array}\right)
$$

Here we use the superscript "c" in the periods to denote them as solutions around the conifold point. We see that one of the solutions $\omega_{1}^{c}$ is singled out as it multiplies the log in the solution $\omega_{3}^{c}$. By a Lefshetz argument [30] it corresponds to the integral over the vanishing $S^{3}$ cycle $B_{1}$ and moreover a solution containing the log is the integral over 
dual cycle $A_{1}$. Comparing with (3.34)3.38) shows in the Type IIA interpretation that the $D 6$ brane becomes massless. To determine the symplectic basis we analytically continue the solutions (3.34) from $\psi=\infty$ and get

$$
\left(\begin{array}{c}
F_{0} \\
F_{1} \\
X_{0} \\
X_{1}
\end{array}\right)=\left(\begin{array}{rrrr}
0 & \frac{\sqrt{5}}{2 \pi i} & 0 & 0 \\
a-\frac{11 i}{2} g & b-\frac{11 i}{2} h & c-\frac{11 i}{2} r & 0 \\
d & e & f & -\frac{\sqrt{5}}{(2 \pi i)^{2}} \\
i g & i h & i r & 0
\end{array}\right)\left(\begin{array}{c}
\omega_{0}^{c} \\
\omega_{1}^{c} \\
\omega_{2}^{c} \\
\omega_{3}^{c}
\end{array}\right)
$$

Six of the real numbers $a, \ldots, r$ are only known numerically 10 . Nevertheless we can give the symplectic form exactly in the new basis

$$
\mathrm{d} F_{k} \wedge \mathrm{d} X_{k}=-\frac{1}{(2 \pi i)^{3}}\left(\frac{5}{2} \mathrm{~d} \omega_{2}^{c} \wedge \mathrm{d} \omega_{0}^{c}+(-5) \mathrm{d} \omega_{3}^{c} \wedge \mathrm{d} \omega_{1}^{c}\right) .
$$

The mirror map should be invariant under the conifold monodromy and vanishing at the conifold. The vanishing period has $D 6$ brane charge and is singled out to appear in the numerator of the mirror map. The numerator is not fixed up to the fact that $\omega_{3}^{c}$ should not appear. The simplest mirror map compatible with symplectic form $(3.72)$ is

$$
\begin{aligned}
t_{D}(\delta) & :=\frac{\omega_{1}^{c}}{\omega_{0}^{c}}=\delta-\frac{3 \delta^{2}}{10}+\frac{11 \delta^{3}}{75}-\frac{9 \delta^{4}}{100}+\frac{5839 t_{D}^{5}}{93750}+\mathcal{O}\left(t_{D}^{6}\right) \\
\delta\left(t_{D}\right) & =t_{D}+\frac{3 t_{D}^{2}}{10}+\frac{t_{D}^{3}}{30}+\frac{t_{D}^{4}}{200}+\frac{169 t_{D}^{5}}{375000}+\mathcal{O}\left(t_{D}^{6}\right)
\end{aligned}
$$

We call this the dual mirror map and denote it $t_{D}$ to distinguish from the large complex structure modulus case.

In the holomorphic limit $\bar{\delta} \rightarrow 0$, the Kahler potential and metric should behave as $e^{-K} \sim \omega_{0}^{c}$ and $G_{\delta \bar{\delta}} \sim \partial_{\delta} t_{D}$. We can find the asymptotic behavior of various generators,

$$
\begin{aligned}
X & =\frac{1}{1-\psi}=-\frac{1}{\delta} \\
A & =-\frac{3}{5}-\frac{2}{25} \delta+\frac{2}{125} \delta^{2}-\frac{52}{9375} \delta^{3}+\mathcal{O}\left(\delta^{4}\right) \\
B & =\frac{6}{625} \delta^{2}-\frac{76}{9375} \delta^{3}+\frac{611}{93750} \delta^{4}+\mathcal{O}\left(\delta^{5}\right) \\
B_{2} & =\frac{12}{625} \delta-\frac{16}{3125} \delta^{2}+\frac{82}{46875} \delta^{3}+\mathcal{O}\left(\delta^{4}\right) \\
B_{3} & =\frac{12}{625}+\frac{28}{3125} \delta-\frac{78}{15625} \delta^{2}+\mathcal{O}\left(\delta^{3}\right)
\end{aligned}
$$

\footnotetext{
${ }^{10} a=6.19501627714957 \ldots, b=1.016604716702582 \ldots, c=-0.140889979448831 \ldots, d=$ $1.07072586843016 \ldots, e=-0.0247076138044847 \ldots, g=1.29357398450411 \ldots, h=\frac{2 b g \pi-(\sqrt{5} d)}{2 a \pi}$, $r=\frac{5+16 c g \pi^{3}}{16 a \pi^{3}}, f=\frac{\sqrt{5} b+8 c d \pi^{2}}{8 a \pi^{2}}$.
} 
Now we can expand

$$
F_{\text {conifold }}^{(g)}=\lim _{\bar{\delta} \rightarrow 0}\left(\omega_{0}^{c}\right)^{2(g-1)}\left(\frac{1-\psi}{\psi}\right)^{g-1} P_{g}
$$

around the conifold point in terms of $t_{D}$ using the dual mirror map (3.73)).

Remarkably it turns out that shifts $\omega_{0}^{c} \rightarrow \omega_{0}^{c}+b_{1} \omega_{1}^{c}+b_{2} \omega_{2}^{c}$ does not affect the structure we are interested in. The fact that the $b_{1}$ shifts do not affect the amplitudes is reminiscent of the $\operatorname{SL}(2, \mathbb{C})$ orbit theorem [59, 60] and is proven in App. A.3. It is therefore reasonable to state the results in the more general polarization and define $\hat{\omega}_{0}^{c}=\omega_{0}^{c}+b_{1} \omega_{1}^{c}+b_{2} \omega_{2}^{c}$. We first determine the genus 0 prepotential checking consistency of the solutions with special geometry. Defining $\hat{t}_{D}=\frac{\omega_{1}^{c}}{\hat{\omega}_{0}^{c}}$ and $\Pi_{c o n}=$ $\left(\hat{\omega}_{0}^{c}, \omega_{1}^{c}, \frac{5}{2} \omega_{2}^{c},-5 \omega_{3}^{c}\right)^{T}$ we get

$$
\hat{\Pi}^{\text {orb }}=\hat{\omega}_{0}\left(\begin{array}{c}
1 \\
\hat{t}_{D} \\
2 F_{\text {conif. }}^{(0)}-\hat{t}_{D} \partial_{\hat{t}_{D}} F_{\text {conif. }}^{(0)} \\
\partial_{\hat{t}_{D}} F_{\text {conif. }}^{(0)}
\end{array}\right) .
$$

We can now calculate the $F^{(g)}$ in the generalized polarization. Since the normalization of $\hat{\omega}_{0}^{c}$ is not fixed by the Picard-Fuchs equation, we pick the normalization $b_{0}=1$ that will be convenient later on 11 Using the known expression for the ambiguity at genus 2, 3 [22, 33, 20] for $g=0-3$ and Castelnuovos bound for $g>3$ we find the same interesting structure first observed in [12]

$$
\begin{aligned}
& F_{\text {conif. }}^{(0)}=-\frac{5}{2} \log \left(\hat{t}_{D}\right) \hat{t}_{D}^{2}+\frac{5}{12}\left(1-6 b_{1}\right) \hat{t}_{D}^{3}+\left(\frac{5}{12}\left(b_{1}-3 b_{2}\right)-\frac{89}{1440}-\frac{5}{4} b_{1}^{2}\right) \hat{t}_{D}^{4}+\mathcal{O}\left(\hat{t}_{D}^{5}\right) \\
& F_{\text {conif. }}^{(1)}=-\frac{\log \left(\hat{t}_{D}\right)}{12}+\left(\frac{233}{120}-\frac{113 b_{1}}{12}\right) \hat{t}_{D}+\left(\frac{233 b_{1}}{120}-\frac{113 b_{1}{ }^{2}}{24}-\frac{107 b_{2}}{12}-\frac{2681}{7200}\right) \hat{t}_{D}^{2}+\mathcal{O}\left(\hat{t}_{D}^{3}\right) \\
& F_{\text {conif. }}^{(2)}=\frac{1}{240 t_{D}^{2}}-\left(\frac{120373}{72000}+\frac{11413 b_{2}}{144}\right)+\left(\frac{107369}{150000}-\frac{120373 b_{1}}{36000}+\frac{23533 b_{2}}{720}-\frac{11413 b_{1} b_{2}}{72}\right) \hat{t}_{D}+\mathcal{O}\left(\hat{t}_{D}^{2}\right) \\
& F_{\text {conif. }}^{(3)}=\frac{1}{1008 \hat{t}_{D}^{4}}-\left(\frac{178778753}{324000000}+\frac{2287087 b_{2}}{43200}+\frac{1084235 b_{2}{ }^{2}}{864}\right)+\mathcal{O}\left(\hat{t}_{D}\right) \\
& F_{\text {conif. }}^{(4)}=\frac{1}{1440 \hat{t}_{D}^{6}}-\left(\frac{977520873701}{3402000000000}+\frac{162178069379 b_{2}}{3888000000}+\frac{5170381469 b_{2}{ }^{2}}{2592000}+\frac{490222589 b_{2}{ }^{3}}{15552}\right)+\mathcal{O}\left(\hat{t}_{D}\right)
\end{aligned}
$$

As explained in sec. 2.2 we expect this gap structure to be present at higher genus as in (1.2), i.e.

$$
F_{\text {conifold }}^{(g)}=\frac{(-1)^{g-1} B_{2 g}}{2 g(2 g-2) \hat{t}_{D}^{2 g-2}}+\mathcal{O}\left(\hat{t}_{D}^{0}\right) .
$$

If this is true then it will impose $2 g-2$ conditions on the holomorphic ambiguity (3.55). We can further note that because the prefactor in (3.76) goes like $\delta^{g-1}$, and

\footnotetext{
${ }^{11}$ The $b_{0}$ dependence can be restored noting that each order in $\hat{t}_{D}$ is homogeneous in $b_{i}$.
} 
the generator $X$ goes like $X \sim \frac{1}{\delta}$, the terms $a_{i} X^{i}$ in holomorphic ambiguity (3.55) with $i \leq g-1$ do not affect the gap structure in (1.2). Therefore the gap structure fixes the coefficients $a_{i}$ of $i=g, \cdots, 3 g-3$ in (3.55), but not the coefficients $a_{i}$ with $i \leq g-1$. Note that the choice of $b_{i}, i=0,1,2$ does not affect the gap structure at all. In App. A.3 it is proven that the generators of the modular forms in $P_{g}$ do not change under the shift $b_{1}$. The effect of this shift is hence merely a Kähler gauge transformation. On the other hand we note that imposing the invariance of the gap structure under the $b_{2}$ shift does not give further conditions on the $a_{i}$, but it does affect the subleading expansion.

\subsection{Fixing the holomorphic ambiguity: a summary of results}

Putting all the information together, let us do a counting of number of unknown coefficients. Originally we have $3 g-2$ coefficients in (3.55). The constant map calculation [35, 37, 39] in A-model large complex structure modulus limit $\psi \sim \infty$ fixes one constant $a_{0}$. The conifold expansion around $\psi \sim 1$ fixes $2 g-2$ coefficients $a_{i}$ of $i=g, \cdots, 3 g-3$, and the orbifold expansion around $\psi \sim 0$ further fixes $\left\lceil\frac{3(g-1)}{5}\right\rceil$ coefficients. So the number of unknown coefficients at genus $g$ is

$$
3 g-2-\left(1+2 g-2+\left\lceil\frac{3(g-1)}{5}\right\rceil\right)=\left[\frac{2(g-1)}{5}\right] .
$$

This number is zero for genus $g=2,3$. So we could have computed the $g=2,3$ topological strings using this information, although the answers were already known. At $g \geq 4$ there are still some unknown constants. However, in the A-model expansion when we rewrite the Gromov-Witten invariants in terms of Gopakumar-Vafa invariants, one can in principle use the Castelnuovos bound to fix the $F^{(g)}$ up to genus 51 . This will be shown in sect. 5.2 .

\section{One-parameter Calabi-Yau spaces with three reg- ular singular points}

We generalize the analysis for the quintic to other one Kähler parameter Calabi-Yau three-folds, whose mirror $W$ has a Picard-Fuchs-system with three regular singular points. Note that this type of CY has been completely classified [56] starting from the Riemann-Hilbert reconstruction of the Picard-Fuchs equations given the monodromies and imposing the special geometry property as well as integrality conditions on the solutions of the latter. There are 13 cases whose mirrors can be realized as hypersurfaces and complete intersections in weighted projective spaces with trivial fundamental group 12 , and are well-known in the mirror symmetry literature. A

\footnotetext{
${ }^{12}$ We also have some results on the cases with non-trivial fundamental group. They are available on request.
} 
fourteenth case is related to a degeneration of a two parameter model as pointed out in [58]. Five are obtained as a free discrete orbifold of the former CY.

We focus on the 13 former cases. In the notation of [33] these complete intersections of degree $\left(d_{1}, d_{2}, \cdots, d_{k}\right)$ in weighted projective spaces $\mathbb{P}^{n}\left(w_{1}, \ldots, w_{l}\right)$, CalabiYau manifolds are abbreviated as $X_{d_{1}, d_{2}, \cdots, d_{k}}\left(w_{1}, \ldots, w_{l}\right)$. For example, the familiar quintic manifold is denoted as $X_{5}\left(1^{5}\right)$. The list of 13 such examples is the following:

$$
\begin{aligned}
& X_{5}\left(1^{5}\right): \vec{a}=\left(\frac{1}{5}, \frac{2}{5}, \frac{3}{5}, \frac{4}{5}\right), X_{6}\left(1^{4}, 2\right): \vec{a}=\left(\frac{1}{6}, \frac{2}{6}, \frac{4}{6}, \frac{5}{6}\right), \quad X_{8}\left(1^{4}, 4\right): \vec{a}=\left(\frac{1}{8}, \frac{3}{8}, \frac{5}{8}, \frac{7}{8}\right), \\
& X_{10}\left(1^{3}, 2,5\right): \vec{a}=\left(\frac{1}{10}, \frac{3}{10}, \frac{7}{10}, \frac{9}{10}\right), \quad X_{3,3}\left(1^{6}\right): \vec{a}=\left(\frac{1}{3}, \frac{1}{3}, \frac{2}{3}, \frac{2}{3}\right), \\
& X_{4,2}\left(1^{6}\right): \vec{a}=\left(\frac{1}{4}, \frac{1}{2}, \frac{1}{2}, \frac{3}{4}\right), \quad X_{3,2,2}\left(1^{7}\right): \vec{a}=\left(\frac{1}{3}, \frac{1}{2}, \frac{1}{2}, \frac{2}{3}\right), \\
& X_{2,2,2,2}\left(1^{8}\right): \vec{a}=\left(\frac{1}{2}, \frac{1}{2}, \frac{1}{2}, \frac{1}{2}\right), \quad X_{4,3}\left(1^{5}, 2\right): \vec{a}=\left(\frac{1}{4}, \frac{1}{3}, \frac{2}{3}, \frac{3}{4}\right), \\
& X_{4,4}\left(1^{4}, 2^{2}\right): \vec{a}=\left(\frac{1}{4}, \frac{1}{4}, \frac{3}{4}, \frac{3}{4}\right), \quad X_{6,2}\left(1^{5}, 3\right): \vec{a}=\left(\frac{1}{6}, \frac{1}{2}, \frac{1}{2}, \frac{5}{6}\right), \\
& X_{6,4}\left(1^{3}, 2^{2}, 3\right): \vec{a}=\left(\frac{1}{6}, \frac{1}{4}, \frac{3}{4}, \frac{5}{6}\right), \quad X_{6,6}\left(1^{2}, 2^{2}, 3^{2}\right): \vec{a}=\left(\frac{1}{6}, \frac{1}{6}, \frac{5}{6}, \frac{5}{6}\right) .
\end{aligned}
$$

The examples satisfy the Calabi-Yau condition $\sum_{i} d_{i}=\sum_{i} w_{i}$ required by the vanishing of the first Chern class. The components of the vector $\vec{a}$ specify the Picard-Fuchs operators for the mirror manifolds with $h^{2,1}=1$,

$$
\left\{\left(\psi \frac{\partial}{\partial \psi}\right)^{4}-\psi^{-1} \prod_{i=1}^{4}\left(\psi \frac{\partial}{\partial \psi}-a_{i}\right)\right\} \Pi=0 .
$$

The indices of the Picard-Fuchs equation satisfy $\sum_{i=1}^{4} a_{i}=2$ and we have arranged $a_{i}$ in increasing order for later convenience.

There are three singular points in the moduli space. The maximally unipotent point is the large complex structure modulus limit $\psi \sim \infty$ that has three logarithmic solutions for the Picard-Fuchs equation. The conifold point is $\psi=1$ with three power series solutions and one logarithmic solution for the Picard-Fuchs equation. If the indices $a_{i}$ are not degenerate, the Picard-Fuchs equation around the orbifold point $\psi=0$ has four powers series solutions with the leading terms going like $\psi^{a_{i}}$. Each degeneration of the indices generates a logarithmic solution for the Picard-Fuchs equation around the orbifold point $\psi=0$.

\subsection{The integration of the anomaly equation}

We can straightforwardly generalize the formalism in [20] to the above class of models. The mirror map is normalized as $t=\log \left(\frac{\prod_{i} d_{i}^{d_{i}}}{\prod_{i} w_{i}^{\omega_{i}}} \psi\right)+\mathcal{O}\left(\frac{1}{\psi}\right)$, so that the classical intersection number in the prepotential is $F^{(0)}=\frac{\kappa}{6} t^{3}+\cdots$ where $\kappa=\frac{\prod_{i} d_{i}}{\prod_{i} w_{i}}$. The 
generators of the topological string amplitudes are defined accordingly,

$$
\begin{aligned}
& A_{p}:=\frac{(\psi \partial \psi)^{p} G_{\psi \bar{\psi}}}{G_{\psi \bar{\psi}}}, \quad B_{p}:=\frac{(\psi \partial \psi)^{p} e^{-K}}{e^{-K}}, \quad(p=1,2,3, \cdots) \\
& C:=C_{\psi \psi \psi} \psi^{3}, \quad X:=\frac{1}{1-\psi} .
\end{aligned}
$$

Here the familiar three point Yukawa coupling is $C_{\psi \psi \psi} \sim \frac{\psi^{-2}}{1-\psi}$, and as in the case of the quintic we denote $A:=A_{1}$ and $B:=B_{1}$. The generators satisfy the derivative relations

$$
\psi \partial_{\psi} A_{p}=A_{p+1}-A A_{p}, \quad \psi \partial_{\psi} B_{p}=B_{p+1}-B B_{p}, \quad \psi \partial_{\psi} X=X(X-1),
$$

and the recursion relations

$$
\begin{aligned}
& B_{4}=\left(\sum_{i} a_{i}\right) X B_{3}-\left(\sum_{i<j} a_{i} a_{j}\right) X B_{2}+\left(\sum_{i<j<k} a_{i} a_{j} a_{k}\right) X B-\left(\prod_{i} a_{i}\right) X \\
& A_{2}=-4 B_{2}-2 A B-2 B+2 B^{2}-2 A+2 X B+X A-r_{0} X-1
\end{aligned}
$$

where the first equation can be derived from the Picard-Fuchs equation (4.81) and the second equation is derived from the special geometry relation up to a holomorphic ambiguity denoted by the constant $r_{0}$. One can fix the constant $r_{0}$ by expanding the generators around any of the singular points in the moduli space. For example, as we will explain, the asymptotic behaviors of various generators around the orbifold point are $A_{p}=\left(a_{2}-a_{1}-1\right)^{p}+\mathcal{O}(\psi), B_{p}=a_{1}^{p}+\mathcal{O}(\psi)$, and $X=1+\mathcal{O}(\psi)$, so we find the constant is

$$
r_{0}=a_{1}\left(1-a_{1}\right)+a_{2}\left(1-a_{2}\right)-1 .
$$

The polynomial topological amplitudes $P_{g}$ are defined by $P_{g}^{(n)}:=C^{g-1} \psi^{n} C_{\psi^{n}}^{(g)}$, and satisfy the recursion relations with initial data

$$
\begin{aligned}
P_{g=0}^{(3)} & =1 \\
P_{g=1}^{(1)} & =\left(\frac{\chi}{24}-2\right) B-\frac{A}{2}+\frac{1}{12}(X-1)+\frac{s_{1}}{2}, \\
P_{g}^{(n+1)} & =\psi \partial_{\psi} P_{g}^{(n)}-\left[n(A+1)+(2-2 g)\left(B-\frac{X}{2}\right)\right] P_{g}^{(n)},
\end{aligned}
$$

where $\chi$ is the Euler character of the Calabi-Yau space and $s_{1}=2 c-\frac{5}{6}$ is a constant that can be fixed by the second Chern class of the Calabi-Yau; see appendix A. We provide the list of constants for the 13 cases of one-parameter Calabi-Yau in the following table.

After changing variables to a convenient basis from $\left(A, B, B_{2}, B_{3}, X\right)$ to $\left(u, v_{1}, v_{2}, v_{3}, X\right)$ as the following:

$$
\begin{aligned}
& B=u, \quad A=v_{1}-1-2 u, \quad B_{2}=v_{2}+u v_{1}, \\
& B_{3}=v_{3}-u v_{2}+u v_{1} X-\left(r_{0}+1\right) u X
\end{aligned}
$$




\begin{tabular}{|r|r|r|r|r|r|}
\hline CY & $X_{5}\left(1^{5}\right)$ & $X_{6}\left(1^{4}, 2\right)$ & $X_{8}\left(1^{4}, 4\right)$ & $X_{10}\left(1^{3}, 2,5\right)$ & $X_{3,3}\left(1^{6}\right)$ \\
\hline$\chi$ & -200 & -204 & -296 & -288 & -144 \\
\hline$s_{1}$ & $\frac{10}{3}$ & $\frac{8}{3}$ & $\frac{17}{6}$ & 2 & $\frac{11}{3}$ \\
\hline $\mathrm{CY}$ & $X_{4,2}\left(1^{6}\right)$ & $X_{3,2,2}\left(1^{7}\right)$ & $X_{2,2,2,2}\left(1^{8}\right)$ & $X_{4,3}\left(1^{5}, 2\right)$ & $X_{4,4}\left(1^{4}, 2^{2}\right)$ \\
\hline$\chi$ & -176 & -144 & -128 & -156 & -144 \\
\hline$s_{1}$ & $\frac{23}{6}$ & $\frac{25}{6}$ & $\frac{9}{2}$ & $\frac{19}{6}$ & $\frac{5}{2}$ \\
\hline $\mathrm{CY}$ & $X_{6,2}\left(1^{5}, 3\right)$ & $X_{6,4}\left(1^{3}, 2^{2}, 3\right)$ & $X_{6,6}\left(1^{2}, 2^{2}, 3^{2}\right)$ & & \\
\hline$\chi$ & -256 & -156 & -120 & & \\
\hline$s_{1}$ & $\frac{7}{2}$ & $\frac{11}{6}$ & 1 & & \\
\hline
\end{tabular}

Table 1: Euler numbers and the constant $s_{1}$

One can follow [20] and use the BCOV formalism to show that $P_{g}$ is a degree $3 g-$ 3 inhomogeneous polynomial of $v_{1}, v_{2}, v_{3}, X$ with the assigned degrees 1,2,3,1 for $v_{1}, v_{2}, v_{3}, X$ respectively. The BCOV holomorphic anomaly equation becomes

$$
\left(\frac{\partial}{\partial v_{1}}-u \frac{\partial}{\partial v_{2}}-u(u+X) \frac{\partial}{\partial v_{3}}\right) P_{g}=-\frac{1}{2}\left(P_{g-1}^{(2)}+\sum_{r=1}^{g-1} P_{r}^{(1)} P_{g-r}^{(1)}\right) .
$$

As in the case of the quintic, the holomorphic anomaly equation determines the polynomial $P_{g}$ up to a holomorphic ambiguity

$$
f=\sum_{i=0}^{3 g-3} a_{i} X^{i}
$$

\subsection{The boundary behaviour}

The constant term $a_{0}$ is fixed by the known constant map contribution in the A-model expansion

$$
F_{\text {A-model }}^{g}=\lim _{t \rightarrow \infty} \omega_{0}^{2(g-1)}\left(\frac{1-\psi}{\kappa \psi}\right)^{g-1} P_{g}
$$

where $\omega_{0}$ is the power series solution in the large complex structure modulus limit.

The main message here is that structure of the conifold expansion is universal. For all cases of Calabi-Yau spaces, the Picard-Fuchs equation around $z=\psi-1$ has four solutions that go like $\omega_{0}=1+\mathcal{O}(z), \omega_{1}=z+\mathcal{O}\left(z^{2}\right), \omega_{2}=z^{2}+\mathcal{O}\left(z^{3}\right)$, and $\omega_{4}=\omega_{1} \log (z)+\mathcal{O}\left(z^{4}\right)$. We define a dual mirror map $t_{D}=\frac{\omega_{1}}{\omega_{0}}=z+\mathcal{O}\left(z^{2}\right)$, expand the topological string amplitudes in terms of $t_{D}$, and impose the following gap structure in the conifold expansion:

$$
\begin{aligned}
F_{\text {conifold }}^{(g)} & =\lim _{\bar{z} \rightarrow 0} \omega_{0}^{2(g-1)}\left(\frac{1-\psi}{\psi}\right)^{g-1} P_{g} \\
& =\frac{(-1)^{g-1} B_{2 g}}{2 g(2 g-2) t_{D}^{2 g-2}}+\mathcal{O}\left(t_{D}^{0}\right) .
\end{aligned}
$$


This fixes $2 g-2$ coefficients in the holomorphic ambiguity.

On the other hand, we discover a rich variety of singularity structures around the orbifold point $\psi=0$. A natural symplectic basis of solutions of the Picard-Fuchs equation (4.81) is picked out by the fractional powers of the leading terms. As in the case of quintic for our purpose we only need the first two solutions $\omega_{0}, \omega_{1}$. The leading behaviors are

1. If $a_{2}>a_{1}$, then we have $\omega_{0}=\psi^{a_{1}}(1+\mathcal{O}(\psi)), \omega_{1}=\psi^{a_{2}}(1+\mathcal{O}(\psi))$.

2. If $a_{2}=a_{1}$, then we have $\omega_{0}=\psi^{a_{1}}(1+\mathcal{O}(\psi)), \omega_{1}=\omega_{0}(\log (\psi)+\mathcal{O}(\psi))$.

In both cases we can define a mirror map around the orbifold point as $s=\frac{\omega_{1}}{\omega_{0}}$. Using the behaviors of Kähler potential $e^{-K} \sim \omega_{0}$ and metric $G_{\psi \bar{\psi}} \sim \partial_{\psi} s$ in the holomorphic limit, we can find the asymptotic expansion of various generators. In both cases $a_{2}>a_{1}$ and $a_{2}=a_{1}$, the leading behaviors are non-singular:

$$
\begin{aligned}
& A_{p}=\left(a_{2}-a_{1}-1\right)^{p}+\mathcal{O}(\psi) \\
& B_{p}=a_{1}^{p}+\mathcal{O}(\psi)
\end{aligned}
$$

This can be used to fix a holomorphic ambiguity (4.84) relating $A_{2}$ to other generators. On the other hand, since the constant (4.84) can be also derived at other singular points of the moduli space, this also serves as a consistency check that we have chosen the correct basis of solutions $\omega_{0}, \omega_{1}$ at the orbifold point.

The orbifold expansion of the topological string amplitudes are

$$
F_{\text {orbifold }}^{(g)}=\lim _{\bar{\psi} \rightarrow 0} \omega_{0}^{2(g-1)}\left(\frac{1-\psi}{\psi}\right)^{g-1} P_{g} \sim \frac{P_{g}}{\psi^{\left(1-2 a_{1}\right)(g-1)}}
$$

We can expand the polynomial $P_{g}$ around the orbifold point $\psi=0$. Generically, $P_{g}$ is power series of $\psi$ starting from a constant term, so $F_{\text {orbifold }}^{(g)} \sim \frac{1}{\psi^{\left(1-2 a_{1}\right)(g-1)}}$. Since $\sum_{i} a_{i}=2$ and $a_{1}$ is the smallest, we know $a_{1} \leq \frac{1}{2}$ and the topological string amplitude around orbifold point is generically singular. Interestingly, we find the singular behavior of the topological strings around the orbifold point is not universal and falls into four classes:

1. This class includes all 4 cases of hypersurfaces and 4 other cases of complete intersections. They are the Calabi-Yau spaces $X_{5}\left(1^{5}\right), X_{6}\left(1^{4}, 2\right), X_{8}\left(1^{4}, 4\right)$, $X_{10}\left(1^{3}, 2,5\right), X_{3,3}\left(1^{6}\right), X_{2,2,2,2}\left(1^{8}\right), X_{4,4}\left(1^{4}, 2^{2}\right), X_{6,6}\left(1^{2}, 2^{2}, 3^{2}\right)$. For these cases the singularity at the orbifold point is cancelled by the series expansion of the polynomial $P_{g}$. The requirement of cancellation of singularity in turn imposes

$$
\left\lceil\left(1-2 a_{1}\right)(g-1)\right\rceil
$$


conditions on the holomorphic ambiguity in $P_{g}$. Taking into account the Amodel constant map condition and the boundary condition from conifold expansion, we find the number of unknown coefficients at genus $g$ is

$$
\left[2 a_{1}(g-1)\right]
$$

Notice for the Calabi-Yau $X_{2,2,2,2}\left(1^{8}\right)$ the cancellation is trivial since in this case $a_{1}=\frac{1}{2}$ and topological strings around the orbifold point are generically non-singular, so this does not impose any boundary conditions.

2. This class of Calabi-Yau spaces include $X_{4,2}\left(1^{6}\right)$ and $X_{6,2}\left(1^{5}, 3\right)$. We find the singularity at the orbifold point is not cancelled by the polynomial $P_{g}$, but it has a gap structure that closely resembles the conifold expansion. Namely, when we expand the topological strings in terms of the orbifold mirror map $s=\frac{\omega_{1}}{\omega_{0}}$, we find

$$
F_{\text {orbifold }}^{(g)}=\frac{C_{g}}{s^{2(g-1)}}+\mathcal{O}\left(s^{0}\right)
$$

where in our normalization convention, the constant is $C_{g}=\frac{B_{2 g}}{2^{5 g-4}(g-1) g}$ for the $X_{4,2}\left(1^{6}\right)$ model, and $C_{g}=\frac{B_{2 g}}{3^{3(g-1)} 4(g-1) g}$ for the $X_{6,2}\left(1^{5}, 3\right)$ model. Since the mirror map goes like $s \sim \psi^{a_{2}-a_{1}}$, and the $P_{g}$ is a power series of $\psi$, this imposes

$$
\left\lceil 2\left(a_{2}-a_{1}\right)(g-1)\right\rceil
$$

conditions on the holomorphic ambiguity in $P_{g}$. In both cases of $X_{4,2}\left(1^{6}\right)$ and $X_{6,2}\left(1^{5}, 3\right)$, we have $a_{2}=\frac{1}{2}$, so the number of un-fixed coefficients is the same as the models in the first class, namely

$$
\left[2 a_{1}(g-1)\right]
$$

3. This class of Calabi-Yau spaces include only the Calabi-Yau $X_{3,2,2}\left(1^{7}\right)$. For this model, the singularity around the orbifold point does not cancel, so there is no boundary condition imposed on the holomorphic ambiguity in $P_{g}$. At genus $g$ we are simply left with $g-1$ unknown coefficients.

4. This class of Calabi-Yau spaces include $X_{4,3}\left(1^{5}, 2\right)$ and $X_{6,4}\left(1^{3}, 2^{2}, 3\right)$. For this class of models, the singularity at the orbifold point is partly cancelled. Specifically, we find $\frac{P_{g}}{\psi^{\left(1-2 a_{1}\right)(g-1)}}$ is not generically regular at $\psi \sim 0$, but $\frac{P_{g}}{\psi^{\left(1-2 a_{2}\right)(g-1)}}$ is always regular. This then imposes

$$
\left\lceil\left(1-2 a_{2}\right)(g-1)\right\rceil
$$

conditions on holomorphic ambiguity in $P_{g}$, and the number of unknown coefficients at genus $g$ is now

$$
\left[2 a_{2}(g-1)\right]
$$


It looks like we have the worst scenarios in the two models $X_{2,2,2,2}\left(1^{8}\right)$ and $X_{3,2,2}\left(1^{7}\right)$, where we essentially get no obvious boundary conditions at the orbifold point $\psi=0$. However after a closer examination, we find some patterns in the leading coefficients of the orbifold expansion as the followings. In our normalization convention, we find the leading constant coefficients of $X_{2,2,2,2}\left(1^{8}\right)$ model is

$$
F_{\text {orbifold }}^{(g)}=\frac{\left(21 \cdot 2^{2 g-2}-5\right)(-1)^{g} B_{2 g} B_{2 g-2}}{2^{2 g-3} g(2 g-2)(2 g-2) !}+\mathcal{O}(\psi)
$$

whereas for $X_{3,2,2}\left(1^{7}\right)$ model the leading coefficients are

$$
F_{\text {orbifold }}^{(g)}=\frac{\left(7 \cdot 2^{2 g-2}-1\right) B_{2 g}}{2^{4 g-3} 3^{2 g-2}(g-1) g} \frac{1}{s^{2 g-2}}+\mathcal{O}\left(\frac{1}{s^{2 g-8}}\right)
$$

These leading coefficients provide one more boundary condition for the models $X_{2,2,2,2}\left(1^{8}\right)$ and $X_{3,2,2}\left(1^{7}\right)$, although this is not much significant at large genus. On the other hand, we observe the leading coefficients of $X_{2,2,2,2}\left(1^{8}\right)$ model are similar to the constant map contribution of Gromov-Witten invariants except the factor of $\left(21 \cdot 2^{2 g-2}-5\right)$, whereas the leading coefficients of $X_{3,2,2}\left(1^{7}\right)$ model are similar to the conifold expansion except the factor of $\left(7 \cdot 2^{2 g-2}-1\right)$. These non-trivial factors can not be simply removed by a different normalization of variables and therefore contain useful information. In fact in the latter case of $X_{3,2,2}\left(1^{7}\right)$ model, the factor of $\left(7 \cdot 2^{2 g-2}-1\right)$ will motivate our physical explanations of the singularity in a moment.

As shown in [33], see also sec. [5, for a fixed genus $g$, the Gopakumar-Vafa invariants $n_{d}^{g}$ are only non-vanishing when the degree $d$ is bigger than $a_{g}$, where $a_{g}$ is a model dependent number with weak genus dependence, in particular for large $g$ one has $d_{\min }-1=a_{g} \sim \sqrt{g}$. So long as the number of zeros in the low degree Gopakumar-Vafa invariants are bigger than the number of unknown coefficients that we determine above using all available boundary conditions, we have a redundancy of data to compute the topological strings recursively genus by genus, and are able to make non-trivial checks of our computations. For all of the 13 cases of one-parameter Calabi-Yau spaces, we are able to push the computation to very high genus. So far our calculations are limited only by the power of our computational facilities.

We now propose a "phenomenological" theory of the singularity structures at the point with rational branching. Our underlying philosophy is that a singularity of $F^{(g)}$ in the moduli space can only be generated if there are charged massless states near this point of moduli space. This is already familiar from the behaviors at infinity $\psi=\infty$ and conifold point $\psi=1$. At infinity $\psi=\infty$ the relevant charged states are massive $D 2-D 0$ brane bound states and therefore $F^{(g)}$ are regular, whereas at the conifold point there is a massless charged state from a $D 3$ brane wrapping a vanishing 3-cycle, and this generates the gap like singularity at the conifold point as we have explained. We should now apply this philosophy to the much richer behavior at the 
orbifold point $\psi=0$. We discuss the four classes of models in the same order as mentioned above.

1. We argue for this class of models the $F^{(g)}$ are regular at the orbifold point because there is no massless charged state. A necessary condition would be that the mirror map parameter $s$ is non-zero at the orbifold point, since as we have learned there are D-branes wrapping cycles whose charge and mass are measured by $s$. This is clear for the complete intersection cases, namely $X_{3,3}\left(1^{6}\right), X_{2,2,2,2}\left(1^{8}\right), X_{4,4}\left(1^{4}, 2^{2}\right), X_{6,6}\left(1^{2}, 2^{2}, 3^{2}\right)$, because for these models the first two indices of the Picard-Fuchs equation is degenerate $a_{2}=a_{1}$, therefore the mirror map goes like

$$
s \sim \log \psi \rightarrow \infty
$$

and we see that the D-branes are very massive and generate exponentially small corrections just like the situation at infinity $\psi=\infty$. As for the hypersurface cases $X_{5}\left(1^{5}\right), X_{6}\left(1^{4}, 2\right), X_{8}\left(1^{4}, 4\right), X_{10}\left(1^{3}, 2,5\right)$, we comment that although naively the mirror map goes like $s \sim \psi^{a_{2}-a_{1}} \rightarrow 0$, there is a change of basis under which the generators are invariant as explained in Appendix A.3, and which could make the periods goes like $\omega_{0} \sim \omega_{1} \sim \psi^{a_{1}}$, therefore the mirror map becomes finite at the orbifold point. This is also consistent with the basis at orbifold point we obtained by analytic continuation from infinity $\psi=\infty$. In fact we checked that the regularity of $F^{(g)}$ is not affected when we take $s$ to be the ratio of generic arbitrary linear combinations of the periods $\omega_{i}, i=0,1,2,3$.

2. We argue this class of models have a conifold like structure because of the same mechanism we have seen for the conifold point $\psi=1$. This is consistent with the fact that the degenerate indices in these cases, e.g. models $X_{4,2}\left(1^{6}\right)$ and $X_{6,2}\left(1^{5}, 3\right)$, are the middle indices, namely we have $a_{2}=a_{3}=\frac{1}{2}$. Therefore the Picard-Fuchs equation constrains one of periods to be a power series proportional to

$$
\omega_{1} \sim \psi^{\frac{1}{2}}
$$

Since there is another period that goes like $\omega_{0} \sim \psi^{a_{1}}$, the mirror map goes like $s \sim \psi^{\frac{1}{2}-a_{1}} \rightarrow 0$ and it is not possible to change the basis in a way such that the mirror map is finite. Integrating out a charged nearly massless particle generates the gap like conifold singularity as we have explained.

3. For the model $X_{3,2,2}\left(1^{7}\right)$ we argue there are two massless states near the orbifold point. Since the middle indices also degenerate $a_{2}=a_{3}=\frac{1}{2}$, we can apply the same reasoning from the previous case and infer that the mirror map goes like $s \sim \psi^{\frac{1}{6}}$ and there must be at least one charged massless state from a D3 brane 
wrapping vanishing 3-cycle. However the situation is now more complicated. We do not find a gap structure in the expansion of $F_{\text {orbifold }}^{(g)}$, and the leading coefficients differ from the usual conifold expansion by a factor of $\left(7 \cdot 2^{2 g-2}-1\right)$ as observed in (4.100). These can be explained by postulating that there are two massless particles in this case whose masses are $m$ and $2 m$. This fits nicely with the $2^{2 g-2}$ power in the intriguing factor of $\left(7 \cdot 2^{2 g-2}-1\right)$, and also explains the absence of gap structure by the possible interactions between the two massless particles.

4. Finally, we discuss the cases of models $X_{4,3}\left(1^{5}, 2\right)$ and $X_{6,4}\left(1^{3}, 2^{2}, 3\right)$. The indices $a_{i}$ are not degenerate in these cases. What makes these models different from the hypersurface cases is the fact that the ratio $\frac{a_{2}}{a_{1}}$ is now not an integer in these cases. This makes it difficult to change the basis such that the mirror map is finite. We conjecture that the mirror map indeed goes like $s \sim \psi^{a_{2}-a_{1}}$ and therefore there exist massless particle(s) whose masses are proportional to $s$ and who are responsible for generating the singularity of $F^{(g)}$ at the orbifold point. This is consistent with our analytic continuation analysis in Appendix A.4. We note that a necessary non-trivial consequence of this scenario would be that the $F_{\text {orbifold }}^{(g)}$ is no more singular than $\frac{1}{s^{2 g-2}}$, i.e. the product

$$
s^{2 g-2} F_{\text {orbifold }}^{(g)} \sim \frac{P_{g}}{\psi^{\left(1-2 a_{2}\right)(g-1)}}
$$

should be regular. This is precisely what we observe experimentally.

\section{Symplectic invariants at large radius}

The coefficients of the large radius expansion of the $\mathcal{F}^{(g)}=\lim _{\bar{t} \rightarrow \infty} F^{(g)}(t, \bar{t})$ have an intriguing conjectural interpretation as symplectic invariants of $M$. First of all we have

$$
\mathcal{F}^{(g)}(q)=\sum_{\beta} r_{\beta}^{(g)} q^{\beta}
$$

where $r_{\beta}^{(g)} \in \mathbb{Q}$ are the Gromow-Witten invariants of holomorphic maps. Secondly the Gopakumar-Vafa invariants [38] count the cohomology of the $D_{0}-D_{2}$ bound state moduli space, see also [33], and are related to the

$$
\begin{aligned}
\mathcal{F}(\lambda, t) & =\sum_{g=0}^{\infty} \lambda^{2 g-2} \mathcal{F}^{(g)}(t) \\
& =\frac{c(t)}{\lambda^{2}}+l(t)+\sum_{g=0}^{\infty} \sum_{\beta \in H_{2}(M, \mathbb{Z})} \sum_{m=1}^{\infty} n_{\beta}^{(g)} \frac{1}{m}\left(2 \sin \frac{m \lambda}{2}\right)^{2 g-2} q^{\beta m} .
\end{aligned}
$$


Here $c(t)$ and $l(t)$ are some cubic and linear polynomials in $t$, which follow from the leading behaviour of $\mathcal{F}^{(0)}$ and $\mathcal{F}^{(1)}$ as explained in (3.37) and (2.20). With $q_{\lambda}=e^{i \lambda}$ we can write a product form 13 for the partition function $Z^{\text {hol }}=\exp \left(\mathcal{F}^{\text {hol }}\right)$

$$
Z_{\mathrm{GV}}^{\mathrm{hol}}(M, \lambda, q)=\prod_{\beta}\left[\left(\prod_{r=1}^{\infty}\left(1-q_{\lambda}^{r} q^{\beta}\right)^{r n_{\beta}^{(0)}}\right) \prod_{g=1}^{\infty} \prod_{l=0}^{2 g-2}\left(1-q_{\lambda}^{g-l-1} q^{\beta}\right)^{(-1)^{g+r}\left({ }^{2 g-2}\right) n_{\beta}^{(g)}}\right]
$$

in terms of the Gopakumar-Vafa invariants $n_{\beta}^{(g)}$. Based on the partition functions there is a conjectural relation of the latter to the Donaldson-Thomas invariants $\tilde{n}_{\beta}^{(g)}$, which are invariants of the moduli space $I_{k}(M, \beta)$ of ideal sheaves $\mathcal{I}$ on $M$. Defining $Z_{\mathrm{DT}}^{\text {hol }}\left(M, q_{\lambda}, q\right)=\sum_{\beta, k \in \mathbb{Z}} \tilde{n}_{\beta}^{(k)} q_{\lambda}^{k} q^{\beta}$ one expects [47]

$$
Z_{\mathrm{GV}}^{\mathrm{hol}}\left(M, q_{\lambda}, q\right) M\left(q_{\lambda}\right)^{\frac{\chi(M)}{2}}=Z_{\mathrm{DT}}^{\mathrm{hol}}\left(M,-q_{\lambda}, q\right),
$$

where the McMahon function is defined as $M\left(q_{\lambda}\right):=\prod_{n \geq 0} \frac{1}{\left(1-q_{\lambda}^{n}\right)^{n}}$. We will give below the information about the $\mathcal{F}^{(g)}$ in terms of the Gopakumar-Vafa invariants and give a more detailed account of the data of the symplectic invariants on the webpage [62].

\subsection{Castelnuovo's theory and the cohomology of the BPS state moduli space}

Let us give checks of the numbers using techniques of algebraic geometry and the description of the BPS moduli space and its cohomology developed in [38, 33. The aim is to check the gap condition in various geometric settings, namely hypersurfaces and complete intersections in (weighted) projective spaces discussed before. According to [38, 33] the BPS number of a given charge, i.e. degree $d$, can be calculated from cohomology of the moduli space $\hat{\mathcal{M}}$ of a $D_{2}-D_{0}$ brane system. The latter is the fibration of the Jacobian $T^{2 \tilde{g}}$ of a genus $\tilde{g}$ curve over its moduli space of deformations $\mathcal{M}$. Curves of arithmetic genus $g<\tilde{g}$ are degenerate curves, in the simplest case with $\delta=\tilde{g}-g$ nodes. Their BPS numbers are calculated using the Euler numbers of relative Hilbert schemes $\mathcal{C}^{(i)}$ of the universal curve $\left(\mathcal{C}^{(0)}=\mathcal{M}, \mathcal{C}^{(1)}\right.$ is the universal curve, etc) in simple situations as follows:

$$
\begin{aligned}
n_{d}^{g} & =n_{d}^{\tilde{g}-\delta}=(-1)^{\operatorname{dim}(\mathcal{M})+\delta} \sum_{p=0}^{\delta} b(\tilde{g}-p, \delta-p) e\left(\mathcal{C}^{(p)}\right), \\
b(g, k) & =\frac{2}{k !}(g-1) \prod_{i=1}^{k-1}(2 g-(k+2)+i), \quad b(g, 0)=0 .
\end{aligned}
$$

As explained in [33] curves in projective spaces meeting the quintic are either plane curves in $\mathbb{P}^{2}$, curves in $\mathbb{P}^{3}$, or $\mathbb{P}^{4}$. In all case one gets from Castelnuovo theory a bound on $g$, which grows for large $d$ like $g(d) \sim d^{2}$. For a detailed exposition of curves

\footnotetext{
${ }^{13}$ Here we dropped the $\exp \left(\frac{c(t)}{\lambda^{2}}+l(t)\right)$ factor of the classical terms at genus 0,1 .
} 


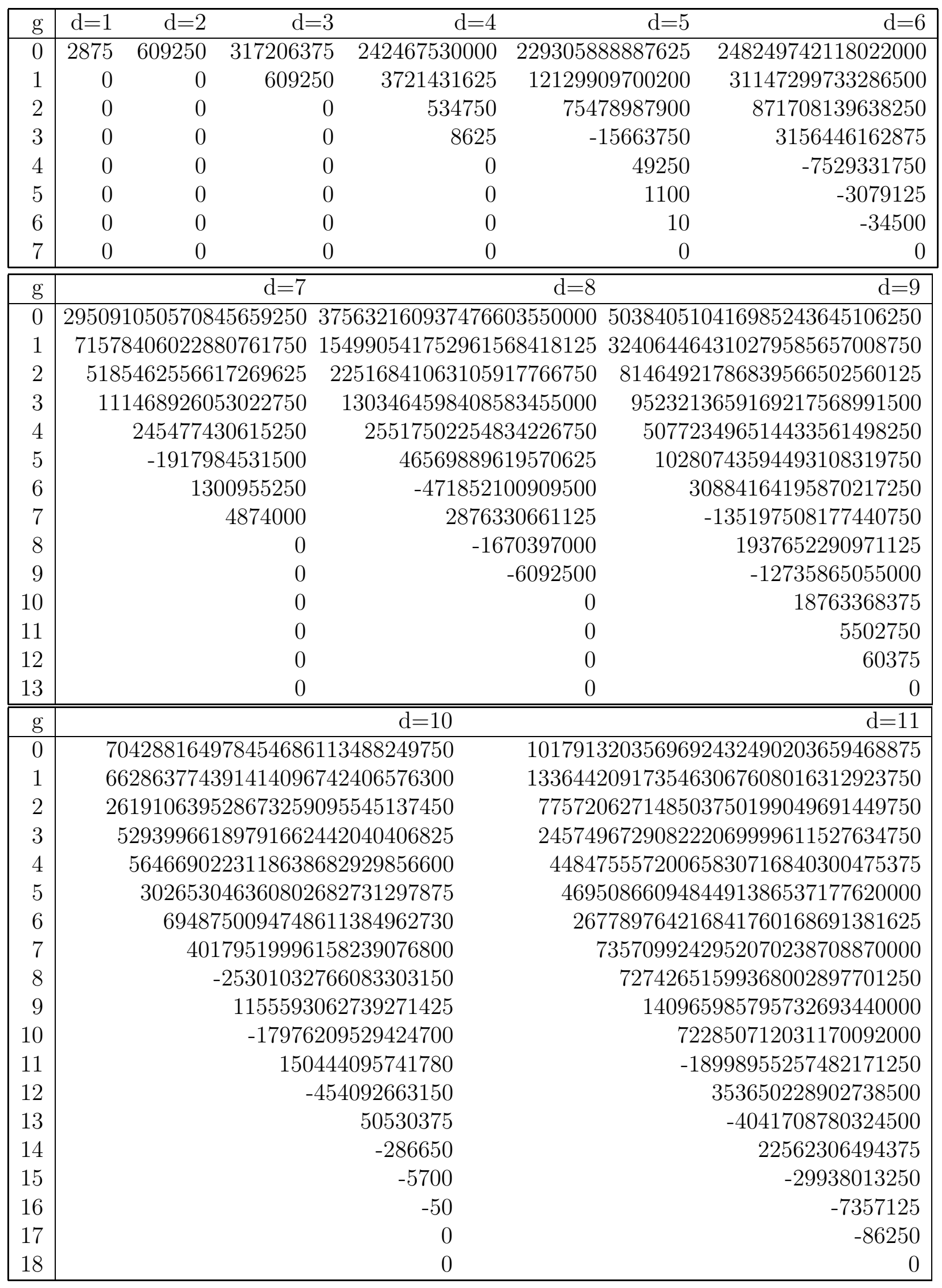

Table 2: BPS invariants $n_{d}^{g}$ on the Quintic hypersurface in $\mathbb{P}^{4}$. See also Table 3. 
in projective space see [61]. Using this information one can determine which curves above is realized and contributes to the BPS numbers. These statements generalize to the hypersurfaces and complete intersections with one Kähler modulus in weighted projective spaces. In particular the qualitative feature $g(d) \sim d^{2}$ of the bound for large $d$ carries over. We note for later convenience that to go from a smooth curve of genus $\tilde{g}$ to a curve with arithmetic genus $g=\tilde{g}-\delta$ by enforcing $\delta$ nodes we get from (5.108)

$$
\begin{aligned}
n_{d}^{\tilde{g}-1} & =(-1)^{\operatorname{dim}(\mathcal{M})+1}(e(\mathcal{C})+(2 \tilde{g}-2) e(\mathcal{M})) \\
n_{d}^{\tilde{g}-2} & =(-1)^{\operatorname{dim}(\mathcal{M})+1}\left(e\left(\mathcal{C}^{(2)}\right)+(2 \tilde{g}-4) e(\mathcal{C})+\frac{1}{2}(2 \tilde{g}-2)(2 \tilde{g}-5) e(\mathcal{M})\right)
\end{aligned}
$$

\subsection{D-branes on the quintic}

One consequence of our global understanding of the $F^{(g)}$ is that we can make detailed statements about the 'number' of $D$-brane states for the quintic at large radius. We focus on $d=5$, because there is a small numerical flaw in the analysis of [33], while the right numerics confirms the gap structure quite significantly. In this case the complete intersection with multidegree $(1,1,5)$ is a plane curve with genus $\tilde{g}=$ $(d-1)(d-2) / 2=6$, while the other possibilities have at most genus $g=2$. Curves of $(g=\tilde{g}, d)=(6,5)$ are therefore smooth plane curves with $\delta=0$ and according to (5.108) their BPS number is simply $n_{d}^{g}=(-1)^{\operatorname{dim} \mathcal{M}} e(\mathcal{M})$. Since $d=5$ their moduli space is simply the moduli space of $\mathbb{P}^{2}$ 's in $\mathbb{P}^{4}, \mathcal{M}$ is the Grassmannian $\mathbb{G}(2,4)$. Grassmannians $\mathbb{G}(k, n)$ have dimensions $(k+1)(n-k)$ and their Euler number can be calculated most easily by counting toric fixed points to be $\chi(\mathbb{G}(k, n))=\left(\begin{array}{l}n+1 \\ k+1\end{array}\right)$. We get $n_{5}^{6}=(-1)^{6} 10=10$.

For the $(g, d)=(5,5)$ curves we have to determine the Euler number of the universal curve $\mathcal{C}$, which is a fibration $\pi: \mathcal{C} \rightarrow \mathcal{M}$ over $\mathcal{M}$. To get an geometric model for $\mathcal{C}$ we consider the projection $\tilde{\pi}: \mathcal{C} \rightarrow X$. The fiber over a point $p \in X$ is the set of $\mathbb{P}^{2}$ 's in $\mathbb{P}^{4}$ which contain the point $p$. This is described as the space of $\mathbb{P}^{1}$ 's in $\mathbb{P}^{3}$ i.e. $\mathbb{G}(1,3)$ with $15 \chi(\mathbb{G}(1,3))=6$. As the fibration $\tilde{\pi}$ is smooth we obtain $e(\mathcal{C})=\chi(X) \chi(\mathbb{G}(1,3))=-200 \cdot 6=-1200$. Applying now (5.109) we get $n_{5}^{5}=(-1)^{5}(-1200+(2 \cdot 6-2) 10)=1100$.

The calculation of $n_{4}^{4}$ requires the calculation of $e\left(\mathcal{C}^{(2)}\right)$. The model for $\mathcal{C}^{(2)}$ is constructed from the fibration $\hat{\pi}: \mathcal{C}^{(2)} \rightarrow \operatorname{Hilb}^{2}(X)$ as follows. A point in $\operatorname{Hilb}^{2}(X)$ are either two distinct points or one point of multiplicity 2 with distinct tangent direction. In both cases the fiber over $P \in \operatorname{Hilb}^{2}(X)$ is an $\mathbb{P}^{2}$ passing though 2 points in $\mathbb{P}^{4}$,

\footnotetext{
${ }^{14}$ The space of $\mathbb{P}^{k}$ 's in $\mathbb{P}^{n}$, which we call $\mathbb{G}(k, n)$, is also the space of $k+1$ complex dimensional subspaces in an $n+1$ dimensional complex vector space, which is often alternatively denote as $G(k+1, n+1)$.

${ }^{15} \mathbb{G}(1,3)$ is Plucker embedded in $\mathbb{P}^{5}$ as a quadric (degree 2 ). From the adjunction formula we also get $\chi(\mathbb{G}(1,3))=6$.
} 
which is a $\mathbb{P}^{2}$. The fibration is smooth and it remains to calculate the Euler number of the basis. There are nice product formulas for the Euler number of symmetric products of surfaces modded out by $S_{n}$. For surfaces it is more cumbersome. We calculate the Euler number $e\left(\operatorname{Sym}^{2}(X)\right)=\left(\begin{array}{c}-199 \\ 2\end{array}\right)$. $\operatorname{Hilb}^{2}(X)$ is the resolution of the orbifold $\operatorname{Sym}^{2}(X)$, which has the diagonal $X$ as fix point set. The resolution replaces each point in the fixed point set by $\mathbb{P}^{2}$. Simple surgery and the smooth fibration structure of $\mathcal{C}^{(2)}$ gives hence $e\left(\mathcal{C}^{(2)}\right)=3\left(e\left(\operatorname{Sym}^{2}(X)+(3-1) e(X)\right)=58500\right.$, which by (5.109) yields $n_{5}^{4}=58500+(2 \cdot 6-4)(-1200)+35 \cdot 10=49250$.

The approach becomes more difficult with the number of free points $\delta$ and at $\delta=4$ it is currently not know how to treat the singularities of the Hilbert scheme.

On the other hand smooth curves at the 'edge' of the Castelnuovo bound are of no principal problem. E.g., using adjunction for a smooth complete intersection of degree $\left(d_{1}, \ldots, d_{r}\right)$ in a (weighted) projective space $\mathbb{W} \mathbb{C P}^{n}\left(w_{1}, \ldots, w_{n+1}\right)$ in Appendix A, we calculate $\chi=(2-2 g)$ and see that the degree 10 genus 16 curve is the complete intersection $(1,2,5)$. The moduli space is calculated by counting the independent deformations of that complete intersection. The degree five constraint lies on the quintic, the linear constraint has five parameters. The identification by the $\mathbb{C}^{*}$ action of the ambient $\mathbb{P}^{4}$ shows that these parameters lie in a $\mathbb{P}^{4}$. This constraint allows to eliminate one variable from the generic quadratic constraint which has hence 10 parameters and a $\mathbb{P}^{9}$ as moduli space. So we check in Tab. 2 the entry $n_{10}^{16}=$ $(-1)^{13} 5 \cdot 10=-50$.

Let us discuss the upper bound on the genus at which we can completely completely fix the $F_{g}$ given simply the bound (3.80). We claim that this bound is $g \leq 51$. At degree 20 there is a smooth complete intersection curve $(1,4,5)$ of that genus. We first check that this is the curve of maximal genus in degree 20. The Castelnuovos bound for curves in $\mathbb{P}^{4}$ shows that they have smaller genus 61. We further see from the discussion in [61] that for curves in $\mathbb{P}^{3}$ not on quadric and a cubic, which would have the wrong degree, the Castelnuovos's bound is saturated for the complete intersection $(1,4,5)$. For $g=51$ (3.80) indicates that the gap, constant map contribution and regularity at the orbifold fixes 131 of the 151 unknown coefficients in (3.55). The vanishing of $n_{d}^{51}=0,1 \leq d \leq 19$ and the value of $n_{20}^{51}=(-1)^{4+34} \chi\left(\mathbb{P}^{4}\right) \chi\left(\mathbb{P}^{34}\right)=165$ for the Euler number of the moduli space of the smooth curve give us the rest of the data. 


\begin{tabular}{|r|rr|}
\hline $\mathrm{d}$ & $\mathrm{g}=18$ & $\mathrm{~g}=19$ \\
\hline$\vdots$ & $\vdots$ & $\vdots$ \\
11 & 0 & 0 \\
12 & -3937166500 & -13403500 \\
13 & 285683687197594125 & -2578098061480250 \\
14 & -95076957496873268057250 & 2730012506820193210000 \\
15 & 6438165666769014564325336250 & -342304337102629200272769700 \\
16 & 15209062594213864261318125134875 & 15209062594213864261318125134875 \\
\hline
\end{tabular}

\begin{tabular}{|r|r|}
\hline $\mathrm{d}$ & $\mathrm{g}=20$ \\
\hline$\vdots$ & $\vdots$ \\
11 & 0 \\
12 & 0 \\
13 & 10690009494250 \\
14 & -59205862559233156250 \\
15 & 15368486208424999875838025 \\
16 & -1036824730393980503709247290500 \\
\hline
\end{tabular}

Table 3: Some higher degree genus 18-20 BPS numbers for the quintic. Note that we can calculate all Donaldson-Thomas invariants for $d=1, \ldots, 12$ exactly.

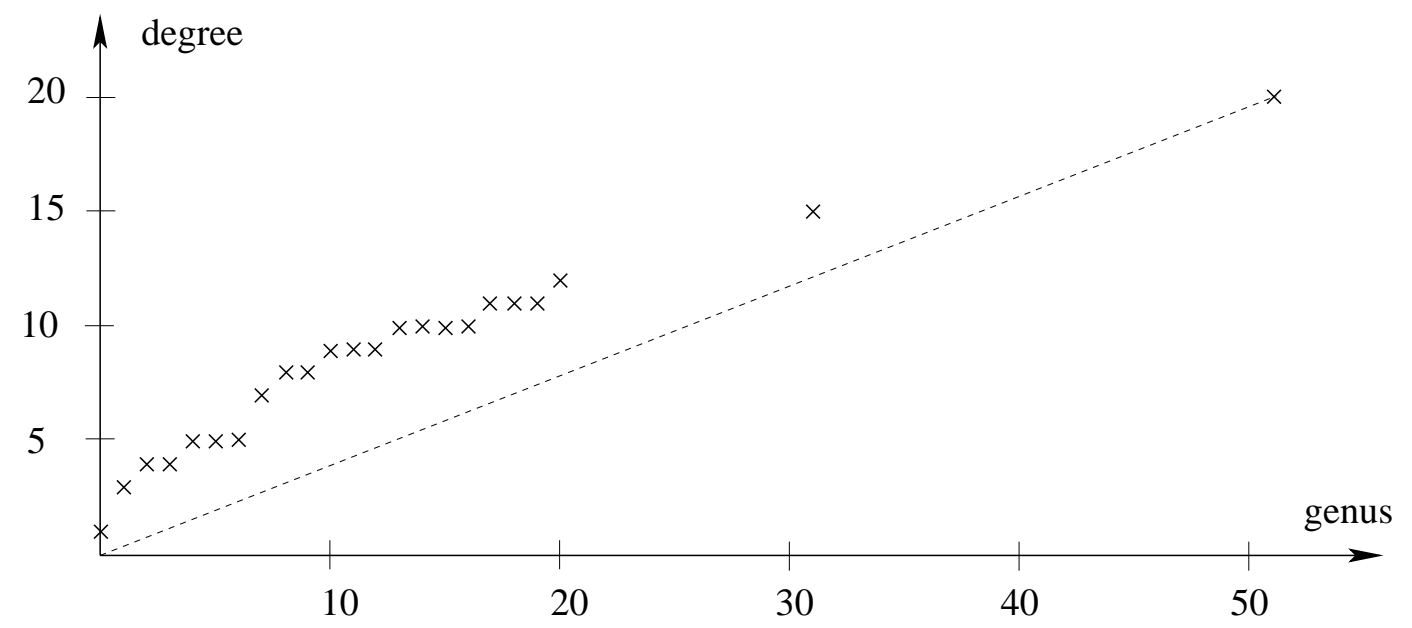

Castelnuovo's bound for higher genus curves on the quintic. The dashed line correspond roughly (up to taking the floor) to the number of coefficients in $f_{g}$ (3.55) which are not fixed by constant map contribution, conifold and orbifold boundary conditions.

It is of course no problem to calculate form the B-model the higher genus amplitudes to arbitrary degree. For completeness we report the first nontrivial numbers for $g=18-20$ in Tab. 3 . 


\subsection{D-brane states on hypersurfaces in weighted projective space}

Similarly, for the sextic in $\mathbb{P}^{4}\left(1^{4}, 2\right)$, the degree $(1,2,6)$ complete intersection curve has genus $g=10$ and degree $d=\frac{\prod_{i} d_{i}}{\prod_{k} w_{k}}=6$ in the weighted projective space . Its moduli space is $\mathbb{P}^{3}$ for the degree one constraint, i.e., we can eliminate $x_{4}$ form the quadric and the 7 coefficeints of the monomials $x_{1}^{2}, x_{1} x_{2}, x_{1} x_{3}, x_{2}^{2}, x_{2} x_{3}, x_{3}^{2}, x_{5}$ form a $\mathbb{P}^{6}$. This yields $n_{6}^{10}=(-1)^{9} 4 \cdot 7=-28$.

There are further checks for the octic in $\mathbb{P}^{4}\left(1^{4}, 4\right)$ BPS invariants. The complete intersection $\left(1^{2}, 8\right)$ has total degree 2 and genus $g=3$. The two linear constraints describe a $\mathbb{P}^{1}$ in $\mathbb{P}^{3}$ i.e. an $\mathbb{G}(1,3)$ with Euler number 6 and dimension 4 , which yields $n_{2}^{3}=6$. Similarly we have a $g=7$ complete intersection $(1,2,8)$ of degree 4 , whose moduli space is $\mathbb{P}^{3}$ times $\mathbb{P}^{5}$ hence $n_{4}^{7}=24$.

For the degree 10 hypersurface in $\mathbb{P}^{4}\left(1^{3}, 2,5\right)$ we check the BPS invariants: From the degree $(1,1,10)$ hypersuface of degree 1 complete intersection with $g=2$. The moduli space of the linear constraints are just the one of point in $\mathbb{P}^{2}$, i.e. $\mathbb{P}^{2}$, hence $n_{1}^{2}=3$. The degree $(1,2,10)$ complete intersection with total degree 2 and genus 4 has the moduli space of the linear constraint, which is $\mathbb{P}^{2}$ and of the quadratic constraint is $\mathbb{P}^{3}$ (from the coefficients of the monomials $x_{1}^{2}, x_{1} x_{2}, x_{2}^{2}, x_{4}$ ), yielding $n_{2}^{4}=-12$. Finally the $(1,3,10)$ complete intersection with genus 7 and degree 3 , has a moduli space $\mathbb{P}^{2}$ times $\mathbb{P}^{5}$ (from the coefficients of the monomials $x_{1}^{3}, x_{1}^{2} x_{2}, x_{1} x_{2}^{2}, x_{1} x_{4}, x_{2}^{3}, x_{2} x_{4}$ ) and $n_{3}^{7}=-18$.

These checks in different geometrical situations establish quite impressively the universality of the gap structure at the conifold expansion.

\subsection{D-branes on complete intersections}

Here we summarize our results on one modulus complete intersections in (weighted) projective space. More complete results are available in [62]. Again we can check many BPS invariants associated to the smooth curves.

Let us check e.g.in table 7 the $n_{9}^{10}=15$. According to A.114 we see that at degree 9 there is a smooth genus 10 curve, given by a complete intersection of multi degree $\left(1^{2}, 3^{2}\right)$ in $\mathbb{P}^{5}$. Their moduli space is the Grassmannian $\mathbb{G}(3,5)$, which has Euler number 15 and dimension 8 , hence $n_{9}^{10}=15$. In a very similar way it can be seen that the $n_{8}^{9}$ comes form a complete intersection curve of degree $\left(4,2,1^{2}\right)$ with the same moduli space, so $n_{8}^{9}=15$ in table 8 . Grassmannians related to complete intersection are also identified with the moduli spaces of the following smooth curves: The total degree six curve of genus seven in table 11 is a CI of multi degree $\left(1^{2}, 3,4\right)$. Its moduli space is a $\mathbb{G}(2,4)$ explaining $n_{6}^{7}=10$. The degree four curve of genus five in table 12 is of multi degree $\left(1^{2}, 4^{2}\right)$ and has moduli space $\mathbb{G}(1,3)$ yielding $n_{4}^{5}=6$. The degree four curve of genus five in table 13 is of multi degree $\left(1^{2}, 2,6\right)$ and has 
moduli space $\mathbb{G}(2,4)$ yielding $n_{4}^{5}=10$. The degree two curve of genus three in table 14 is of multi degree $\left(1^{2}, 4,6\right)$ and has moduli space $\mathbb{G}(0,2)=\mathbb{P}^{2}$ yielding $n_{2}^{3}=3$. The moduli space of the degree 4 genus seven curve $(1,2,4,6)$ is an $\mathbb{P}^{2}$ times the moduli space $\mathbb{P}^{4}$ of quadrics in $\mathbb{W} \mathbb{C} \mathbb{P}^{3}\left(1^{2}, 2^{2}\right)$, so that $n_{4}^{7}=(-1)^{6} 3 \cdot 5=15$. For the degree $(6,6)$ complete intersection in $\mathbb{W} \mathbb{C P}^{3}\left(1^{2}, 2^{2}, 3^{2}\right)$, see table 15 , we have a degree one genus two intersection $\left(1^{2}, 6^{2}\right)$, whose moduli space is a point hence $n_{1}^{2}=1$, a degree two genus four intersection $\left(1,2,6^{2}\right)$, whose moduli space is $\mathbb{P}^{1}$ times the moduli space $\mathbb{P}^{2}$ of quadrics in $\mathbb{W} \mathbb{C} \mathbb{P}^{2}\left(1,2^{2}\right)$ hence $n_{2}^{4}=-6$, a degree three genus seven intersection $\left(1,3,6^{2}\right)$, whose moduli space is $\mathbb{P}^{1}$ times the moduli space $\mathbb{P}^{4}$ of cubics in $\mathbb{W} \mathbb{C P}^{4}\left(1,2^{2}, 3^{2}\right)$ hence $n_{3}^{7}=-10$ and finally a degree four genus eleven intersection $\left(1,4,6^{2}\right)$, whose moduli space is $\mathbb{P}^{1}$ times the moduli space $\mathbb{P}^{5}$ of quadrics in $\mathbb{W} \mathbb{C P}^{4}\left(1,2^{2}, 3^{2}\right)$ hence $n_{4}^{11}=12$.

There are many further checks that are somewhat harder to perform. E.g. we notice that there is a genus one degree three curve in the $(3,2,2)$ CI in $\mathbb{P}^{6}$, which comes from a complete intersection $\left(1^{4}, 3\right)$. Now the moduli space of this complete intersection in $\mathbb{P}^{6}$ is $\mathbb{G}(2,6)$. However not all $\mathbb{P}^{2}$ parametrized by $\mathbb{G}(2,6)$, which contain the cubic, are actually in the two quadrics of the $(3,2,2)$ CICY. We can restrict to those $\mathbb{P}^{2}$, which fulfill these constraints, by considering the simultaneous zeros of sections of two rank six bundles of quadratic forms on the moving $\mathbb{P}^{2}$. These are a number of points, which is calculated by the integral of the product of the Chern classes of these rank 6 bundles over $\mathbb{G}(2,6)$. Indeed we obtain, for example with "Schubert" 65.

$$
n_{3}^{1}=(-1)^{0} \int_{\mathbb{G}(2,6)} c_{6}^{2}(\operatorname{Sym}(2, Q))=64,
$$

which confirms the corresponding entry in table 9 .

\section{Conclusions}

In this paper we solve the topological string B-model on compact Calabi-Yau $M$ using the modularity of the $F_{g}$, the wave function transformation property of $Z$ and the boundary information imposed by effective action considerations. The method pushes the calculation to unprecedented high genus amplitudes. E.g. for the quintic the boundary condition count (3.80) together with the simplest vanishing arguments at large volume fixes the amplitudes up to genus $g=51$. Beyond that the prime mathematical problem to overcome in this region of the moduli space is understand the degeneration of more then four points in the relative Hilbert scheme of the universal curves in a threefold 16 . Similar problems have been encountered in [66], where

\footnotetext{
${ }^{16}$ As a motivation and check for the task to develop the theory of Hilbert schemes for 3folds we calcultated the invariants explicitly to high genus. For the quintic to genus 20 and for all other the results up genus 12 are available at 62 .
} 
it was suggested to fix a very similar ambiguity to a anholomorphic $\mathrm{SL}(2, \mathbb{Z})$-modular elliptic index of a $D 4-D 2-D 0$ brane system [66, 67, 68]. There one uses $\mathrm{SL}(2, \mathbb{Z})$ invariance of the index and a dual dilute gas approximation in $A d S_{3} \times S^{2} \times M$ to fix the coeffcients of the ring of modular forms. The construction of the moduli-space of the $D 4-D 2-D 0$ brane system uses rational GW invariants and implies non-trivial relations among them [66]. Such considerations could in principal provide further boundary conditions at large radius.

Our sharpest tool is the global control of $Z$ over $\mathcal{M}(M)$ and we expect that by a closer analysis of the RR-spectrum at the orbifold of compact Calabi-Yau, we will be able to recover at least the $\left\lceil\frac{2}{d}(g-1)\right\rceil$ conditions that one loses relative to the local cases [13] and solve the model completely. We obtained not only the Gromov-Witten, the Donaldson-Thomas and Gopakumar-Vafa invariants at infinity, but also the local expansion at the conifold, the Gepner point and other more exotic singularities with one or more massless states. The leading singular terms in the effective action reflect the massless states. The branch locus of the 13 parameter models has an intriguing variety of such light spectra and we can learn from the effective action about the singularity and vice versa. Stability properties of theses states have been analysed in App. A.4.

Most importantly our exact expansions do contain further detailed information of the towers massive RR-states at these points. We described them in natural local variables. The information from different genera should be of great value for the study of stable even D-brane bound states on compact Calabi-Yau as it is the content of the supersymmetric index of [38], which is protected under deformations of the complex structure. Non-compact Calabi-Yau such as the resolution of $\mathbb{C}^{n} / G$, with $G \in \operatorname{SL}(n, \mathbb{C})$ have no complex moduli. The issue does not arise and the situation is better understood, see e.g. [63, 64] for reviews.

One can also use the explicit expansions to study the integrable theories that have been associated to the local expansion of the topological string on Calabi-Yau manifold, such as the $c=1$ string at the conifold or the quiver gauge theories at the orbifold, matrix models e.g. at the ADE singularities and new ones for more exotic singularities such as the branch points of the complete intersections CalabiYau manifolds that we discussed here.

The ability to obtain the imprint of the BPS spectrum on the effective action everywhere on the moduli space is of phenomenological interest as flux compactifications drive the theory to attractor points inside the moduli space.

Our expression are governed by the representation of the modular group of the Calabi-Yau on almost holomorphic forms, which we explicitly constructed from the periods, without having much of an independent theory about them. The simpler case of the torus suggest that such forms and their extensions should play a role in the study of of virtually any physical amplitude - open or closed — in compactifications 
on the Calabi-Yau space, even as conjectured in the hypermultiplet sector 69.

One may finally wonder whether the topological string B-model is an integrable theory that is genuinely associated to this new and barely explored class of modular forms on Calabi-Yau spaces moduli spaces, whereas most known integrable models are associated to abelian varieties. As it was noted in [48, 15, 49] in the complex moduli space extended by the dilaton, called extended phase space, one has one has rigid special Kähler geometry and many aspects of the sympletic transformations and its metaplectic realization are easier understood in the extended phase space. There are two maps $\Phi^{(i)}: \mathcal{M} \rightarrow T_{I J}^{(k)}, I, J=1, \ldots, \frac{h^{3}}{2}$ from the complex moduli space to tensors in the extended phase space on which $\left(\begin{array}{cc}A & B \\ C & D\end{array}\right) \in \operatorname{Sp}\left(h^{3}, \mathbb{Z}\right)$ acts projectively like $T^{(k)} \mapsto\left(A T^{(k)}+B\right)\left(C T^{k}+D\right)^{-1}$. For the holomorphic object $\tau_{I J}=$ $\partial_{I} \partial_{J} F^{(0)}=: T_{I J}^{(1)}(t)$, which is mostly discussed in this context of the metaplectic transfomations [48, 15, 49], $\operatorname{Im}(\tau)$ is indefinite, while for the non-holomorphic object $\mathcal{N}_{I J}=\bar{\tau}_{I J}+2 i \frac{\operatorname{Im} \tau_{I K} X^{K} \operatorname{Im} \tau_{I L} X^{L}}{X^{L} \operatorname{Im} \tau_{K L} X^{L}}=: T_{I J}^{(2)}(t, \bar{t})$ comes from the kinetic term in the $10 \mathrm{~d}$ action whose reduction involves the Hodge-star on $M$. It's imaginary part $\operatorname{Im}(\mathcal{N})>0$ is the kinetic term for the vector multiplets and is hence positive definite. In other words $\operatorname{Im} \Phi^{(2)}$ defines a map to the Siegel upper space. $\Phi^{(2)}$ should relate Siegel modular forms for admittedly very exotic subgroups [50] of $\operatorname{Sp}(4, \mathbb{Z})$ to Calabi-Yau amplitudes. Such Siegel modular forms for abelian varieties are also associated to $N=2$ Seiberg-Witten (gauge) theories, while the modular forms on Calabi-Yau studied in this paper underline $N=2$ exact terms in $N=2$ supergravity. The map $\Phi^{(2)}$ could be a manifestation of a gravity-gauge theory correspondence for $4 \mathrm{~d}$ theories with $N=2$ supersymmetry.

It is no principal problem to generalize this to multi-moduli Calabi-Yau as long as the Picard-Fuchs equations are known. These have different, more general singularities with interesting local effective actions. In K3 fibrations which have at least two moduli, the modular properties are much better understood and in fact the ambiguity in the fiber is complete fixed heterotic string calculations. Moreover these cases have $N=2$ field theory limits, which contain further information, which might be sufficient to solve these models [40].

\section{Acknowledgments:}

We thank M. Aganagic, V. Bouchard, T. Grimm, S. Katz, M. Kontsevich, M. Marino, C. Vafa, S. T. Yau and D. Zagier for discussions. Sheldon Katz helped us with the verifications of the BPS numbers and Cumrun Vafa with remarks on the draft. Don Zagier's comments on [12] triggered many ideas here. We thank the MSRI in Berkeley and AK thanks in particular the Simons Professorship Program. MH/AK thank also the Simons Workshops in Mathematics and Physics 05/06 for its hospitality. 


\section{A Appendices}

\section{A.1 Classical intersection calculations using the adjunction formula}

The adjunction formula 17 for the total Chern class of a for dimension $m=n-r$ smooth complete intersections $M$ of multi degree $d_{1}, \ldots, d_{r}$ in a weighted projective space $\mathbb{W} \mathbb{C P}^{n}\left(w_{1}, \ldots, w_{n+1}\right)$ is

$$
c\left(T_{M}\right)=\sum_{i} c_{i}\left(T_{M}\right)=\frac{c\left(T_{\mathbb{W} \mathbb{C P}}\right)}{c(\mathcal{N})}=\frac{\prod_{i=1}^{n+1}\left(1+w_{i} K\right)}{\prod_{k=1}^{r}\left(1+d_{k} K\right)}=\sum_{i} c_{i} K^{i},
$$

where $c\left(T_{\mathbb{W} \mathbb{P} P}\right)=\sum_{i} c_{i}\left(T_{\mathbb{W} \mathbb{C P}}\right)=\prod_{i=1}^{n+1}\left(1+w_{i} K\right)$ is the total Chern class of the weighted projective space, $K$ is its Kähler class and $c(\mathcal{N})=\prod_{k=1}^{r}\left(1+d_{k} K\right)$ is the total Chern class of the normal bundle.

Integration of a top form $\omega=x J^{m}$ with $J=\left.K\right|_{M}$ over $M$ is obtained by integration along the normal direction as

$$
\int_{M} \omega=\int_{\mathbb{W} \mathbb{C P}} \omega \wedge c_{r}(\mathcal{N})=\frac{x}{\prod_{k=1}^{n+1} w_{k}} \prod_{k=1}^{r} d_{k}
$$

Here we used the normalization $\int_{\mathbb{W} \mathbb{C P}} K^{n}=\frac{1}{\prod_{k=1}^{n+1} w_{k}}$. This yields the first line below:

$$
\begin{aligned}
\kappa & =\int_{M} J^{m}=\frac{\prod_{k=1}^{r} d_{k}}{\prod_{i=1}^{r+1} w_{i}} \\
\chi & =\int_{M} c_{3}\left(T_{M}\right)=\frac{c_{3}}{\prod_{k=1}^{n+1} w_{k}} \prod_{k=1}^{r} d_{k} \\
c & =\frac{1}{24} \int_{M} c_{2} \wedge J=\frac{1}{24} \frac{c_{2}}{\prod_{k=1}^{n+1} w_{k}} \prod_{k=1}^{r} d_{k} \\
a & =\frac{1}{2} \int_{M} i_{*} c_{1}(D) \wedge J=\frac{1}{2} \int_{\mathbb{W} \mathbb{C P}} \frac{c\left(T_{M}\right)}{(1+J)} \wedge J^{r+1}=\frac{\operatorname{coeff}\left(\frac{c\left(T_{M}\right)}{(1+J)}, J^{m-1}\right)_{(A} \cdot \prod_{k=1}^{n+1} w_{k}}{2 \cdot 11}
\end{aligned}
$$

Combining (A.111,A.112) one gets the line 2 and 3. The leading $t$ terms in $F_{0}$ can be obtained by calculating $Z(M)$ using (3.38), while the last line follows from the calculation of $Z(D)$ assuming that the $D_{4}$-brane is supported on $D$ the restriction of the hyperplane clas: 18 of $\mathbb{W} \mathbb{C P}$ to $M$ and the Gysins formula for smooth embeddings [29].

\footnotetext{
${ }^{17}$ See 29 for a pedagogical account of these matters.

${ }^{18} a$ is physically less relevant, as it does not affect the effective action. Its value $a=\frac{11}{2}$ obtained for the quintic from (A.116) checks with [19]
} 


\begin{tabular}{|r|rrrrr|}
\hline $\mathrm{g}$ & $\mathrm{d}=1$ & $\mathrm{~d}=2$ & $\mathrm{~d}=3$ & $\mathrm{~d}=4$ & $\mathrm{~d}=5$ \\
\hline 0 & 7884 & 6028452 & 11900417220 & 34600752005688 & 24595034333130080 \\
1 & 0 & 7884 & 145114704 & 1773044322885 & 17144900584158168 \\
2 & 0 & 0 & 17496 & 10801446444 & 571861298748384 \\
3 & 0 & 0 & 576 & -14966100 & 1985113680408 \\
4 & 0 & 0 & 6 & -47304 & -21559102992 \\
5 & 0 & 0 & 0 & 0 & 22232340 \\
6 & 0 & 0 & 0 & 0 & 63072 \\
7 & 0 & 0 & 0 & 0 & 0 \\
\hline
\end{tabular}

\begin{tabular}{|r|rrr|}
\hline $\mathrm{g}$ & $\mathrm{d}=6$ & $\mathrm{~d}=7$ & $\mathrm{~d}=8$ \\
\hline 0 & 513797193321737210316232672190432091294474925211284058913384803271372834984 \\
1 & 1476647364569529236041197243574587406496495592 & 9381487423491392389034886369 \\
2 & 13753100019804005556 & 233127389355701229349884 & 3246006977306701566424657380 \\
3 & 411536108778637626 & 19655355035815833642912 & 561664896373523768591774196 \\
4 & 1094535956564124 & 628760082868148062854 & 48641994707889298118864544 \\
5 & -18316495265688 & 3229011644338336680 & 1863506489926528403683191 \\
6 & 207237771936 & -18261998133124302 & 20758968356323626025164 \\
7 & -583398600 & 513634614205788 & 10040615124628834206 \\
8 & -146988 & -8041642037676 & 1129699628821681740 \\
9 & -3168 & 54521267292 & -38940584273866593 \\
10 & -28 & -43329384 & 904511824896888 \\
11 & 0 & -110376 & -12434437188576 \\
12 & 0 & 0 & 76595605884 \\
\hline
\end{tabular}

Table 4: BPS invariants $n_{d}^{g}$ on the Sextic hypersurface in $\mathbb{P}^{4}\left(1^{4}, 2\right)$.

\section{A.2 Tables of Gopakumar-Vafa invariants}

We list the tables of BPS invariants for all the Calabi-Yau models computed in this paper. 


\begin{tabular}{|r|rrrrr|}
\hline $\mathrm{g}$ & $\mathrm{d}=1$ & $\mathrm{~d}=2$ & $\mathrm{~d}=3$ & $\mathrm{~d}=4$ & $\mathrm{~d}=5$ \\
\hline 0 & 29504 & 128834912 & 1423720546880 & 23193056024793312 & 467876474625249316800 \\
1 & 0 & 41312 & 21464350592 & 1805292092705856 & 101424054914016355712 \\
2 & 0 & 864 & -16551744 & 12499667277744 & 5401493537244872896 \\
3 & 0 & 6 & -177024 & -174859503824 & 20584473699930496 \\
4 & 0 & 0 & 0 & 396215800 & -674562224718848 \\
5 & 0 & 0 & 0 & 301450 & 12063928269056 \\
6 & 0 & 0 & 0 & 4152 & -86307810432 \\
7 & 0 & 0 & 0 & 24 & 37529088 \\
8 & 0 & 0 & 0 & 0 & 354048 \\
$\vdots$ & $\vdots$ & $\vdots$ & $\vdots$ & $\vdots$ & \\
\hline
\end{tabular}

Table 5: BPS invariants $n_{d}^{g}$ on the Octic hypersurface in $\mathbb{P}^{4}\left(1^{4}, 4\right)$.

\begin{tabular}{|c|rrrr|}
\hline $\mathrm{g}$ & $\mathrm{d}=1$ & $\mathrm{~d}=2$ & $\mathrm{~d}=3$ & $\mathrm{~d}=4$ \\
\hline 0 & 231200 & 12215785600 & 1700894366474400 & 350154658851324656000 \\
1 & 280 & 207680960 & 161279120326840 & 103038403740897786400 \\
2 & 3 & -537976 & 1264588024791 & 8495973047204168640 \\
3 & 0 & -1656 & -46669244594 & 61218893443516800 \\
4 & 0 & -12 & 630052679 & -2460869494476896 \\
5 & 0 & 0 & -1057570 & 145198012290472 \\
6 & 0 & 0 & -2646 & -5611087226688 \\
7 & 0 & 0 & -18 & 125509540304 \\
8 & 0 & 0 & 0 & -1268283512 \\
$\vdots$ & $\vdots$ & $\vdots$ & $\vdots$ & $\vdots$ \\
\hline
\end{tabular}

Table 6: BPS invariants $n_{d}^{g}$ on the degree 10 hypersurface in $\mathbb{P}^{4}\left(1^{3}, 2,5\right)$. 


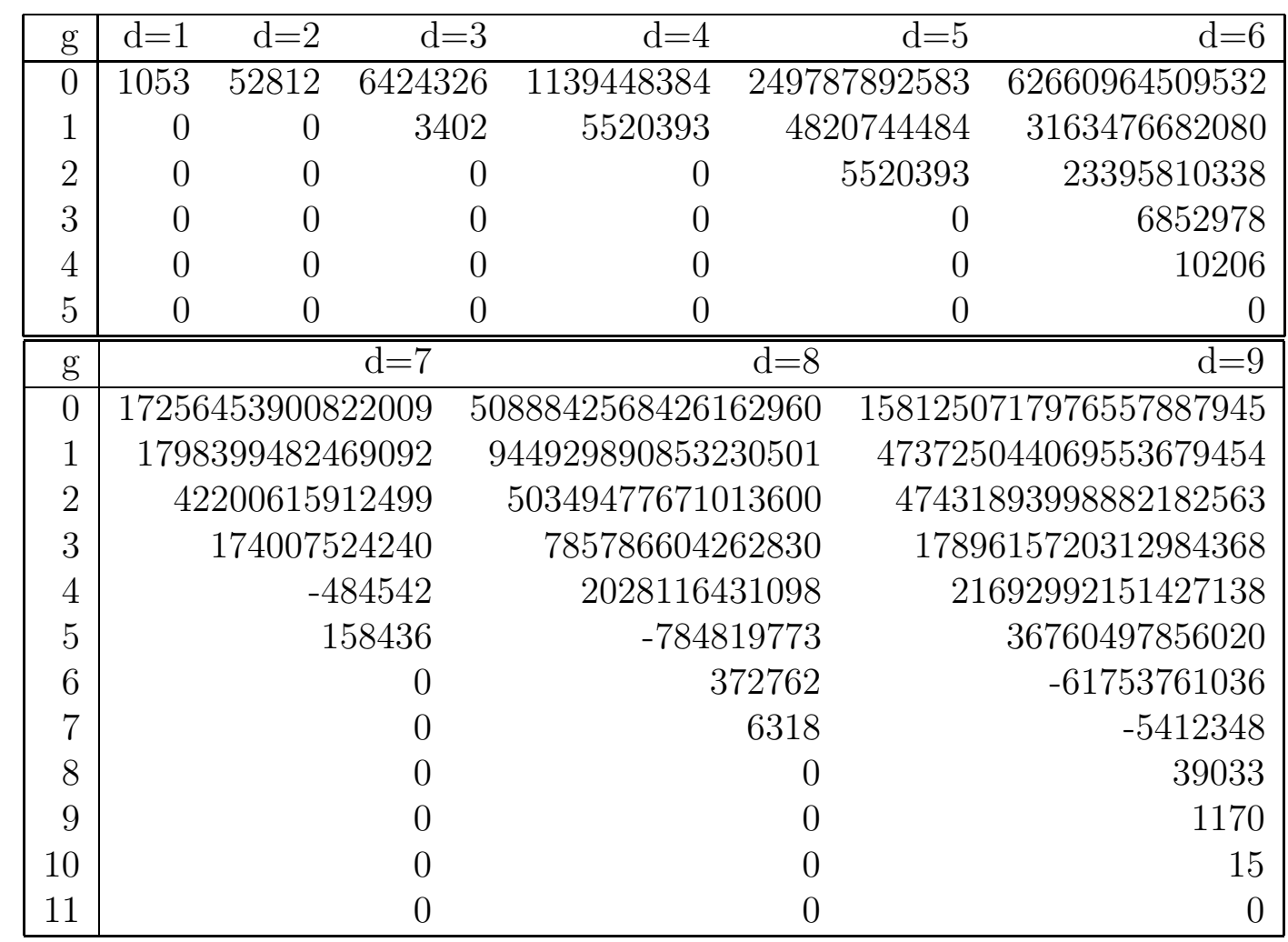

Table $7: n_{d}^{g}$ for the degree $(3,3)$ complete intersection in $\mathbb{P}^{5}$.

\begin{tabular}{|c|c|c|c|c|c|c|c|}
\hline $\mathrm{g}$ & $\mathrm{d}=1$ & $\mathrm{~d}=2$ & \multicolumn{2}{|c|}{$\mathrm{d}=3$} & $\mathrm{~d}=4$ & $\mathrm{~d}=5$ & $\mathrm{~d}=6$ \\
\hline 0 & 1280 & 92288 & \multicolumn{2}{|c|}{15655168} & 3883902528 & \multicolumn{2}{|c|}{$1190923282176 \quad 417874605342336$} \\
\hline 1 & 0 & 0 & & 0 & 16 & \multicolumn{2}{|c|}{$16069888 \quad 174937485184$} \\
\hline 2 & 0 & 0 & & 0 & -8 & 7680 & 12679552 \\
\hline 3 & 0 & 0 & & 0 & 0 & 0 & 276864 \\
\hline 4 & 0 & 0 & & 0 & 0 & 0 & 0 \\
\hline $\mathrm{g}$ & \multicolumn{4}{|c|}{$\mathrm{d}=7$} & & $\mathrm{~d}=8$ & $\mathrm{~d}=9$ \\
\hline 0 & \multicolumn{3}{|c|}{160964588281789696} & \multicolumn{2}{|c|}{66392895625625639488} & \multicolumn{2}{|c|}{28855060316616488359936} \\
\hline 1 & \multicolumn{3}{|c|}{19078577926517760} & & 088192680381290336 & \multicolumn{2}{|c|}{9895851364631438617600} \\
\hline 2 & \multicolumn{3}{|c|}{494602061689344} & & 853657285175383648 & \multicolumn{2}{|c|}{1137794220513866498304} \\
\hline 3 & \multicolumn{3}{|c|}{2016330670592} & & 14859083841009280 & \multicolumn{2}{|c|}{49286012311292922368} \\
\hline 4 & \multicolumn{3}{|c|}{-285585152} & & 37334304102560 & \multicolumn{2}{|c|}{679351051885623552} \\
\hline 5 & \multicolumn{3}{|r|}{591360} & & -46434384200 & \multicolumn{2}{|r|}{1103462757073920} \\
\hline 6 & \multicolumn{3}{|r|}{7680} & \multicolumn{2}{|r|}{-8285120} & \multicolumn{2}{|r|}{-4031209095680} \\
\hline 7 & \multicolumn{3}{|r|}{0} & \multicolumn{2}{|r|}{67208} & \multicolumn{2}{|r|}{370290688} \\
\hline 8 & \multicolumn{3}{|r|}{0} & \multicolumn{2}{|r|}{1520} & \multicolumn{2}{|r|}{-2270720} \\
\hline 9 & \multicolumn{3}{|r|}{0} & \multicolumn{2}{|r|}{15} & \multicolumn{2}{|r|}{-25600} \\
\hline 10 & \multicolumn{3}{|r|}{0} & \multicolumn{2}{|r|}{0} & & 0 \\
\hline
\end{tabular}

Table 8: $n_{d}^{g}$ for the degree $(4,2)$ complete intersection in $\mathbb{P}^{5}$. 


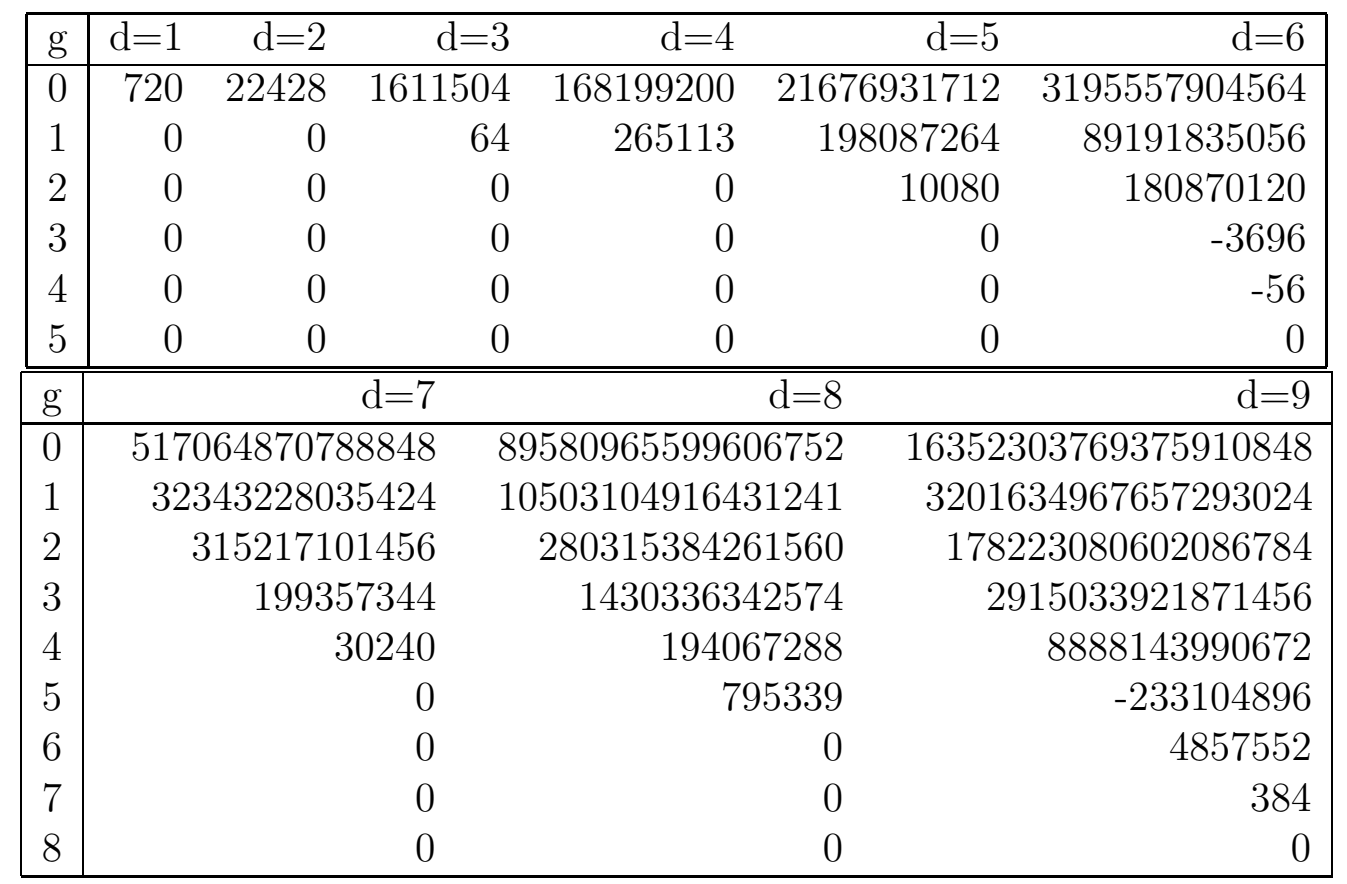

Table 9: $n_{d}^{g}$ for the $(3,2,2)$ complete intersection in $\mathbb{P}^{6}$.

\begin{tabular}{|r|rrrrrrr|}
\hline $\mathrm{g}$ & $\mathrm{d}=1$ & $\mathrm{~d}=2$ & $\mathrm{~d}=3$ & $\mathrm{~d}=4$ & $\mathrm{~d}=5$ & $\mathrm{~d}=6$ & $\mathrm{~d}=7$ \\
\hline 0 & 512 & 9728 & 416256 & 25703936 & 1957983744 & 170535923200 & 16300354777600 \\
1 & 0 & 0 & 0 & 14752 & 8782848 & 2672004608 & 615920502784 \\
2 & 0 & 0 & 0 & 0 & 0 & 1427968 & 2440504320 \\
3 & 0 & 0 & 0 & 0 & 0 & 0 & 0 \\
4 & 0 & 0 & 0 & 0 & 0 & $\mathrm{~d}=9$ & 0 \\
\hline \hline $\mathrm{g}$ & $\mathrm{d}=8$ & $\mathrm{~d}=10$ & $\mathrm{~d}=11$ \\
\hline 0 & 1668063096387072179845756064329728202064979838915548162347339059011866069504 \\
1 & 123699143093152 & 22984995833484288 & 4071465816864581632 & 698986176207439627264 \\
2 & 1628589698304 & 702453851520512 & 236803123487243776 & 68301097513852719616 \\
3 & 2403984384 & 4702943495168 & 4206537025629952 & 2482415474680798208 \\
4 & -37632 & 2449622016 & 16316531089408 & 29624281509824512 \\
5 & -672 & 258048 & 2777384448 & 73818807399424 \\
6 & 0 & 0 & 0 & 4283904 & 1153891840 \\
7 & 0 & 0 & 0 & 0 \\
8 & 0 & 0 & 0 & \\
\hline
\end{tabular}

Table 10: $n_{d}^{g}$ for the degree $(2,2,2,2)$ complete intersection in $\mathbb{P}^{7}$. 


\begin{tabular}{|r|rrrrrr|}
\hline $\mathrm{g}$ & $\mathrm{d}=1$ & $\mathrm{~d}=2$ & $\mathrm{~d}=3$ & $\mathrm{~d}=4$ & $\mathrm{~d}=5$ & $\mathrm{~d}=6$ \\
\hline 0 & 1944 & 223560 & 64754568 & 27482893704 & 14431471821504 & 8675274727197720 \\
1 & 0 & 27 & 161248 & 381704265 & 638555324400 & 891094220317561 \\
2 & 0 & 0 & 0 & 227448 & 3896917776 & 20929151321496 \\
3 & 0 & 0 & 0 & 81 & 155520 & 75047188236 \\
4 & 0 & 0 & 0 & 0 & 5832 & -40006768 \\
5 & 0 & 0 & 0 & 0 & 0 & 26757 \\
6 & 0 & 0 & 0 & 0 & 0 & 816 \\
7 & 0 & 0 & 0 & 0 & 0 & 10 \\
8 & 0 & 0 & 0 & 0 & 0 & 0 \\
\hline
\end{tabular}

Table 11: $n_{d}^{g}$ for the degree $(4,3)$ complete intersection in $\mathbb{W} \mathbb{C} \mathbb{P}^{5}\left(1^{5}, 2\right)$.

\begin{tabular}{|r|rrrrrr|}
\hline $\mathrm{g}$ & $\mathrm{d}=1$ & $\mathrm{~d}=2$ & $\mathrm{~d}=3$ & $\mathrm{~d}=4$ & $\mathrm{~d}=5$ & $\mathrm{~d}=6$ \\
\hline 0 & 3712 & 982464 & 683478144 & 699999511744 & 887939257620352 & 1289954523115535040 \\
1 & 0 & 1408 & 6953728 & 26841854688 & 88647278203648 & 266969312909257728 \\
2 & 0 & 0 & 3712 & 148208928 & 2161190443904 & 17551821510538560 \\
3 & 0 & 0 & 0 & -12432 & 7282971392 & 362668189458048 \\
4 & 0 & 0 & 0 & 384 & -14802048 & 773557598272 \\
5 & 0 & 0 & 0 & 6 & -22272 & -7046285440 \\
6 & 0 & 0 & 0 & 0 & 0 & 6367872 \\
7 & 0 & 0 & 0 & 0 & 0 & 11264 \\
8 & 0 & 0 & 0 & 0 & 0 & 0 \\
\hline
\end{tabular}

Table 12: $n_{d}^{g}$ for the degree $(4,4)$ complete intersection in $\mathbb{W} \mathbb{C} \mathbb{P}^{5}\left(1^{4}, 2^{2}\right)$

\begin{tabular}{|r|rrrrrr|}
\hline $\mathrm{g}$ & $\mathrm{d}=1$ & $\mathrm{~d}=2$ & $\mathrm{~d}=3$ & $\mathrm{~d}=4$ & $\mathrm{~d}=5$ & $\mathrm{~d}=6$ \\
\hline 0 & 4992 & 2388768 & 2732060032 & 4599616564224 & 9579713847066240 & 22839268002374163616 \\
1 & 0 & -504 & 1228032 & 79275664800 & 633074010435840 & 3666182351842338408 \\
2 & 0 & -4 & 14976 & -13098688 & 3921835430016 & 128614837503143532 \\
3 & 0 & 0 & 0 & 87376 & -5731751168 & 482407033529880 \\
4 & 0 & 0 & 0 & 1456 & -7098624 & -3978452463012 \\
5 & 0 & 0 & 0 & 10 & -59904 & 1776341072 \\
6 & 0 & 0 & 0 & 0 & 0 & 18680344 \\
7 & 0 & 0 & 0 & 0 & 0 & -7176 \\
8 & 0 & 0 & 0 & 0 & 0 & -36 \\
9 & 0 & 0 & 0 & 0 & & 0 \\
\hline
\end{tabular}

Table 13: $n_{d}^{g}$ for the degree $(6,2)$ complete intersection in $\mathbb{W} \mathbb{C} \mathbb{P}^{5}\left(1^{5}, 3\right)$. 


\begin{tabular}{|r|rrrrr|}
\hline $\mathrm{g}$ & $\mathrm{d}=1$ & $\mathrm{~d}=2$ & $\mathrm{~d}=3$ & $\mathrm{~d}=4$ & $\mathrm{~d}=5$ \\
\hline 0 & 15552 & 27904176 & 133884554688 & 950676829466832 & 8369111295497240640 \\
1 & 8 & 258344 & 5966034472 & 126729436388624 & 2512147219945401752 \\
2 & 0 & 128 & 36976576 & 4502079839576 & 264945385369932352 \\
3 & 0 & 3 & -64432 & 15929894952 & 9786781718701824 \\
4 & 0 & 0 & -48 & -272993052 & 42148996229312 \\
5 & 0 & 0 & 0 & 800065 & -592538522344 \\
6 & 0 & 0 & 0 & 1036 & 14847229472 \\
7 & 0 & 0 & 0 & 15 & -148759496 \\
8 & 0 & 0 & 0 & 0 & 160128 \\
9 & 0 & 0 & 0 & 0 & 96 \\
10 & 0 & 0 & 0 & 0 & 0 \\
\hline
\end{tabular}

Table 14: $n_{d}^{g}$ for the degree $(6,4)$ complete intersection in $\mathbb{W} \mathbb{C P} \mathbb{P}^{5}\left(1^{3}, 2^{2}, 3\right)$.

\begin{tabular}{|r|rrrr|}
\hline $\mathrm{g}$ & $\mathrm{d}=1$ & $\mathrm{~d}=2$ & $\mathrm{~d}=3$ & $\mathrm{~d}=4$ \\
\hline 0 & 67104 & 847288224 & 28583248229280 & 1431885139218997920 \\
1 & 360 & 40692096 & 4956204918600 & 616199133098321280 \\
2 & 1 & 291328 & 254022248925 & 102984983365762128 \\
3 & 0 & -928 & 1253312442 & 6925290146728800 \\
4 & 0 & -6 & -39992931 & 104226246583368 \\
5 & 0 & 0 & 867414 & -442845743788 \\
6 & 0 & 0 & -1807 & 53221926192 \\
7 & 0 & 0 & -10 & -3192574724 \\
8 & 0 & 0 & 0 & 111434794 \\
9 & 0 & 0 & 0 & -1752454 \\
10 & 0 & 0 & 0 & 3054 \\
11 & 0 & 0 & 0 & 12 \\
12 & 0 & 0 & 0 & 0 \\
\hline
\end{tabular}

Table 15: $n_{d}^{g}$ for the degree $(6,6)$ complete intersection in $\mathbb{W} \mathbb{C P}^{5}\left(1^{2}, 2^{2}, 3^{2}\right)$. 


\section{A.3 Invariance of the generators under a change of the basis}

We have seen the topological strings can be written as polynomials of the generators $v_{1}, v_{2}, v_{3}$, and $X$. In the holomorphic limit, these generators can be computed from the first two solutions $\omega_{0}, \omega_{1}$ of the Picard-Fuchs equation. In the followings we prove that under an arbitrary linear change of basis of $\omega_{0}$ and $\omega_{1}$, these generators and therefore the topological strings are actually invariant. This is true anywhere in the moduli space. In particular, this partly explains why the gap structure in the conifold expansion is not affected by a change of basis of $\omega_{0}$ as we observed in all cases.

Since $X=\frac{1}{1-\psi}$ is independent of the basis $\omega_{0}$ and $\omega_{1}$, it is trivially invariant. In the holomorphic limit, The Kahler potential and metric go like $e^{-K} \sim \omega_{0}$ and $G_{\psi \bar{\psi}} \sim \partial_{\psi} t$, where $t=\frac{\omega_{1}}{\omega_{0}}$ is the mirror map. The generators $u$ and $v_{i}$ are related to $A_{i}$ and $B_{i}$, which we recall were defined as

$$
A_{p}:=\frac{(\psi \partial \psi)^{p} G_{\psi \bar{\psi}}}{G_{\psi \bar{\psi}}}, \quad B_{p}:=\frac{(\psi \partial \psi)^{p} e^{-K}}{e^{-K}}, \quad(p=1,2,3, \cdots)
$$

So a different normalization of basis $\omega_{0}$ of $\omega_{1}$, as well as a change of basis in $\omega_{1} \rightarrow \omega_{1}+$ $b_{1} \omega_{0}$ obviously do not change the generators $A_{i}$ and $B_{i}$, and therefore the generators $u$ and $v_{i}$ are also invariant.

We now tackle the remaining less trivial situation, namely a change of basis in $\omega_{0}$ as the following

$$
\omega_{0} \rightarrow \tilde{\omega}_{0}=\omega_{0}+b_{1} \omega_{1}
$$

where $b_{1}$ is an arbitrary constant. We denote the Kähler potential, metric, mirror map and various generators in the new basis by a tilde symbol. It is straightforward to relate them to variables in the original basis. We find the following relations for the mirror map

$$
\begin{gathered}
s=\frac{\omega_{1}}{\tilde{\omega}_{0}}=\frac{t}{1+b_{1} t} \\
\partial_{\psi} s=\frac{\partial_{\psi} t}{\left(1+b_{1} t\right)^{2}}
\end{gathered}
$$

and the generators $A$ and $B$

$$
\begin{aligned}
\tilde{A} & =\frac{\psi \partial_{\psi} \tilde{G}_{\psi \bar{\psi}}}{\tilde{G}_{\psi \bar{\psi}}}=A-\frac{2 b_{1} \psi \partial_{\psi} t}{1+b_{1} \psi} \\
\tilde{B} & =\frac{\psi \partial_{\psi} \tilde{\omega}_{0}}{\tilde{\omega}_{0}}=B+\frac{b_{1} \psi \partial_{\psi} t}{1+b_{1} \psi}
\end{aligned}
$$

So we find the generators $A$ and $B$, as well as the generator $u=B$ are not invariant under the change of basis (A.118). However, we recall the generator $v_{1}$ is defined as

$$
v_{1}=1+A+2 B
$$


Using the equations in (A.120) we find the generator $v_{1}$ is invariant, namely $\tilde{v}_{1}=v_{1}$. To see $v_{2}$ and $v_{3}$ are invariant, we use the derivative relations

$$
\begin{aligned}
& \psi \partial_{\psi} v_{1}=-v_{1}^{2}-2 v_{2}-\left(1+r_{0}\right) X+v_{1} X \\
& \psi \partial_{\psi} v_{2}=-v_{1} v_{2}+v_{3}
\end{aligned}
$$

where $r_{0}$ is a constant that appears in the relation of generator $A_{2}$ to lower generators. These derivative relations are exact and independent of the choice of the basis in asymptotic expansion. We have show that $v_{1}$ and $X$ in the first equations (A.122) are invariant under a change of the basis (A.118), therefore the generator $v_{2}$ appearing on the right hand side must be also invariant. Applying the same logic to the second equation (A.123) we find the generator $v_{3}$ are also invariant.

Our proof explains why a change of basis like (A.118) does not change the gap structure around the conifold point and seems to be related to the $S L_{2}$ orbit theorem of [59, 60]. Under a change of basis, the mirror map at the conifold point is $\tilde{t}_{D}=\frac{\omega_{0} t_{D}}{\tilde{\omega}_{0}}$, and has the asymptotic leading behavior $\tilde{t}_{D} \sim t_{D} \sim \mathcal{O}(\psi)$. Recall in the holomorphic limit, the conifold expansion is

$$
F_{\text {conifold }}^{(g)}=\omega_{0}^{2(g-1)}\left(\frac{1-\psi}{\psi}\right)^{g-1} P_{g}\left(v_{1}, v_{2}, v_{3}, X\right)
$$

As we have shown the generators $v_{i}$ and therefore $P_{g}$ are invariant, so in the new basis

$$
\tilde{F}_{\text {conifold }}^{(g)}=\left(\frac{\tilde{\omega}_{0}}{\omega_{0}}\right)^{2(g-1)} F_{\text {conifold }}^{(g)}=\left(\frac{t_{D}}{\tilde{t}_{D}}\right)^{2(g-1)} F_{\text {conifold }}^{(g)}
$$

It is clear if there is a gap structure in one basis $F_{\text {conifold }}^{(g)}=\frac{(-1)^{g-1} B_{2 g}}{2 g(2 g-2) t_{D}^{2 g-2}}+\mathcal{O}\left(t_{D}^{0}\right)$, the same gap structure will be also present in the other basis,

$$
\tilde{F}_{\text {conifold }}^{(g)}=\frac{(-1)^{g-1} B_{2 g}}{2 g(2 g-2) \tilde{t}_{D}^{2 g-2}}+\mathcal{O}\left(\tilde{t}_{D}^{0}\right)
$$

The asymptotic expansion in sub-leading terms $\mathcal{O}\left(t_{D}^{0}\right)$ and $\mathcal{O}\left(\tilde{t}_{D}^{0}\right)$ will be different and can be computed by the relation between $t_{D}$ and $\tilde{t}_{D}$.

Around the conifold point there is another power series solution to the PicardFuchs equation that goes like $\omega_{2} \sim \mathcal{O}\left(\psi^{2}\right)$. We also observe that the gap structure is not affected by a change of the basis

$$
\omega_{0} \rightarrow \omega_{0}+b_{2} \omega_{2}
$$

It appears to be much more difficult to prove this observation, since now the generators $v_{i}$ are not invariant under this change of basis. A proof of our observation would depend on the specific details of the polynomial $P_{g}$, and probably requires a deeper conceptual understanding of the conifold expansion. We shall leave this for future investigation. 


\section{A.4 Symplectic basis, vanishing cycles and massless particles}

We can study in more details the analytic continuation of the symplectic basis of the periods to the orbifold point $\psi=0$. For the four hypersurface cases and two other complete intersection models $X_{4,3}\left(1^{5}, 2\right)$ and $X_{6,2}\left(1^{3}, 2^{2}, 3\right)$, the indices $a_{i}(i=$ $1,2,3,4)$ of the Picard-Fuchs equation are not degenerate at the orbifold point, so there are 4 power series with the leading behavior of $\psi^{a_{i}}$, and the analytic continuation procedure is similar to the quintic case. In our physical explanation of the singularity structure of higher genus topological string amplitudes in these models, we claim that for the hypersurface cases there is no stable massless charged state around the orbifold point, whereas for models $X_{4,3}\left(1^{5}, 2\right)$ and $X_{6,2}\left(1^{3}, 2^{2}, 3\right)$ there are nearly massless charged states of mass $m \sim \psi^{a_{2}-a_{1}}$. Since the charge and the mass of a D-brane wrapping cycle is determined by the mirror map parameter, which is the ratio of two symplectic periods, it is only possible to have massless particles if there is a rational linear combination of the periods with the leading behavior of $\psi^{a_{k}}, k>1$.

For a complete intersection of degree $\left(d_{1}, \cdots, d_{r}\right)$ in weighted projective space $\mathbb{W} \mathbb{C P}^{n}\left(w_{1}, \cdots, w_{n+1}\right)$ with non-degenerate indices $a_{k}$, the natural basis of solutions at the orbifold point is

$$
\omega_{k}^{\text {orb }}=\psi^{a_{k}} \frac{\prod_{i=1}^{r} \Gamma\left(d_{i} a_{k}\right)}{\prod_{i=1}^{n+1} \Gamma\left(w_{i} a_{k}\right)} \sum_{n=0}^{\infty}\left(c_{0} \psi\right)^{n} \frac{\prod_{i=1}^{n+1} \Gamma\left(w_{i}\left(n+a_{k}\right)\right)}{\prod_{i=1}^{r} \Gamma\left(d_{i}\left(n+a_{k}\right)\right)}, \quad k=1,2,3,4
$$

where $c_{0}=\frac{\prod_{i=1}^{r} d_{i}^{d_{i}}}{\prod_{i=1}^{n+1} w_{i}^{w_{i}}}$. We can write the sum of the series as a contour integra 19 enclosing the positive real axis and analytically continue to the negative real axis to relate the above basis of solutions to the known symplectic basis at infinity $\psi=\infty$. We find the following relation, generalizing the result for quintic case,

$$
\begin{aligned}
\omega_{k}^{\text {orb }}= & (2 \pi i)^{4} \frac{\prod_{i=1}^{r} \Gamma\left(d_{i} a_{k}\right)}{\prod_{i=1}^{n+1} \Gamma\left(w_{i} a_{k}\right)}\left\{\frac{\alpha_{k} F_{0}}{1-\alpha_{k}}-\frac{\alpha_{k} F_{1}}{\left(1-\alpha_{k}\right)^{2}}+\frac{\alpha_{k}\left[\kappa\left(1+\alpha_{k}\right)-2 a\left(1-\alpha_{k}\right)\right]}{2\left(1-\alpha_{k}\right)^{3}} X_{1}\right. \\
& \left.+\frac{\alpha_{k}\left[12 c\left(1-\alpha_{k}\right)^{2}+\kappa\left(1+4 \alpha_{k}+\alpha_{k}^{2}\right)\right]}{6\left(1-\alpha_{k}\right)^{4}} X_{0}\right\}
\end{aligned}
$$

here $\alpha_{k}=\exp \left(2 \pi i a_{k}\right)$ and $\kappa, c, a$ are from the classical intersection calculations in Appendix A.1. We find for all cases the symplectic form and Kahler potential have the same diagonal behavior as the case of the quintic

$$
\omega=d F_{k} \wedge d X_{k}=s_{1} d \omega_{1}^{\text {orb }} \wedge d \omega_{4}^{\text {orb }}+s_{2} d \omega_{2}^{\text {orb }} \wedge d \omega_{3}^{\text {orb }}
$$

and $e^{-K}=\sum_{k=1}^{4} r_{k} \omega_{k}^{\text {orb }} \overline{\omega_{k}^{\text {orb }}}$, for some constants $s_{1}, s_{2}$ and $r_{k}$.

It is straightforward to invert the transformation (A.129) and study the asymptotic behavior of the geometric symplectic basis $\left(F_{0}, F_{1}, X_{0}, X_{1}\right)$ around the orbifold

\footnotetext{
${ }^{19}$ Further useful properties of the periods of the one parameter models have been established in [57.
} 
point. Generically a linear combination of the symplectic periods $\left(F_{0}, F_{1}, X_{0}, X_{1}\right)$ is proportional to the power of $\psi$ with the lowest index, namely, for generic coefficients $c_{1}, c_{2}, c_{3}, c_{4}$ we have

$$
c_{1} X_{0}+c_{2} X_{1}+c_{3} F_{1}+c_{4} F_{0} \sim \omega_{1}^{\text {orb }} \sim \psi^{a_{1}}
$$

In order for massless particles to appear at the orbifold point, there must be a $S p(4, \mathbb{Z})$ transformation of the symplectic basis such that one of periods goes to zero faster that the generic situation, namely we should have integer coefficients $n_{k} \in \mathbb{Z}$ satisfying

$$
n_{1} X_{0}+n_{2} X_{1}+n_{3} F_{1}+n_{4} F_{0} \sim \omega_{2}^{\text {orb }} \sim \psi^{a_{k}}, \quad k>1
$$

We find (A.132) is impossible for three of the hypersurface cases $X_{5}\left(1^{5}\right), X_{8}\left(1^{4}, 4\right)$ and $X_{10}\left(1^{3}, 2,5\right)$, but possible for the Sextic hypersurface $X_{6}\left(1^{4}, 2\right)$ and the complete intersection cases $X_{4,3}\left(1^{5}, 2\right)$ and $X_{6,2}\left(1^{3}, 2^{2}, 3\right)$. Specifically, for the Sextic hypersurface $X_{6}\left(1^{4}, 2\right)$, the condition for (A.132) with $k=2$

$$
n_{1}+3 n_{3}=0, \quad 3 n_{1}+4\left(n_{2}+n_{4}\right)=0
$$

whereas for the complete intersections $X_{4,3}\left(1^{5}, 2\right)$ and $X_{6,2}\left(1^{3}, 2^{2}, 3\right)$, the conditions for (A.132) are

$$
n_{1}+3 n_{3}=0, \quad n_{1}=4 n_{2}+8 n_{4}
$$

and

$$
n_{1}+2 n_{3}=0, \quad n_{2}+\frac{7}{24} n_{3}+n_{4}=0
$$

respectively.

This fact that there are massless integer charged states possible in the models $X_{4,3}\left(1^{5}, 2\right)$ and $X_{4,3}\left(1^{5}, 2\right)$ is entirely consistent with our physical picture, which explained the singular behaviour of the $F^{(g)}$ form the effective action point of view. What is very interesting is the fact that the period degeneration at the branch point of the hypersurface models, $X_{5}\left(1^{5}\right), X_{6}\left(1^{4}, 2\right), X_{8}\left(1^{4}, 4\right)$ and $X_{10}\left(1^{3}, 2,5\right)$ is at genus zero very similar to the cases $X_{4,3}\left(1^{5}, 2\right)$ and $X_{6,2}\left(1^{3}, 2^{2}, 3\right)$. In particular the periods of the models have no logarithmic singularities. The leading behaviour of the higher genus expansion, which we obtain from the global properties, indicates that the BPS states, which are possibly massless by (A.133, A.134,A.135), are stable in $X_{4,3}\left(1^{5}, 2\right)$ and $X_{6,2}\left(1^{3}, 2^{2}, 3\right)$ models, but not stable in the $X_{6}\left(1^{4}, 2\right)$ model.

\section{References}

[1] A. Klemm and E. Zaslow, "Local mirror symmetry at higher genus," arXiv:hep-th/9906046. 
[2] M. Aganagic, A. Klemm, M. Marino and C. Vafa, "The topological vertex," Commun. Math. Phys. 254, 425 (2005) arXiv:hep-th/0305132.

[3] E. Witten, "Chern-Simons Gauge Theory As A String Theory," Prog. Math. 133, 637 (1995) arXiv:hep-th/9207094.

[4] R. Gopakumar and C. Vafa, "On the gauge theory/geometry correspondence," Adv. Theor. Math. Phys. 3, 1415 (1999) arXiv:hep-th/9811131.

[5] R. Dijkgraaf and C. Vafa, "Matrix models, topological strings, and supersymmetric gauge theories," Nucl. Phys. B 644, 3 (2002) arXiv:hep-th/0206255.

[6] M. Aganagic, R. Dijkgraaf, A. Klemm, M. Marino and C. Vafa, "Topological strings and integrable hierarchies," Commun. Math. Phys. 261, 451 (2006) arXiv:hep-th/0312085.

[7] C. Vafa, "Two dimensional Yang-Mills, black holes and topological strings," arXiv:hep-th/0406058.

[8] K. Costello, "Topological conformal field theories and Calabi-Yau categories," math.QA/0412149.

[9] M. Kontsevich, "TQFTs and geometry of pre-Frobenius manifolds" and " $A_{\infty}$ categories and their (co)homology theories," Lecures at the "Erwin Schroedinger Institute," Vienna 2006.

[10] H. Ooguri, A. Strominger and C. Vafa, "Black hole attractors and the topological string," Phys. Rev. D 70, 106007 (2004) arXiv:hep-th/0405146.

[11] H. Ooguri, C. Vafa and E. P. Verlinde, "Hartle-Hawking wave-function for flux compactifications," Lett. Math. Phys. 74, 311 (2005) arXiv:hep-th/0502211.

[12] M. x. Huang and A. Klemm, "Holomorphic anomaly in gauge theories and matrix models," arXiv:hep-th/0605195.

[13] M. x. Huang and A. Klemm, to appear.

[14] N. Seiberg and E. Witten, "Electric - magnetic duality, monopole condensation, and confinement in $\mathrm{N}=2$ supersymmetric Yang-Mills theory," Nucl. Phys. B 426, 19 (1994) [Erratum-ibid. B 430, 485 (1994)] [arXiv:hep-th/9407087].

[15] M. Aganagic, V. Bouchard and A. Klemm, "Topological strings and (almost) modular forms," arXiv:hep-th/0607100.

[16] S. Hosono, M. H. Saito and A. Takahashi, Adv. Theor. Math. Phys. 3 (1999) 177 arXiv:hep-th/9901151]. 
[17] S. Hosono, M. H. Saito and A. Takahashi, arXiv:math.ag/0105148.

[18] R. Dijkgraaf, "Mirror Symmetry and elliptic curves," in The moduli Space of Curves, Progr. Math. 129, 149; K. Kaneko and D. B. Zagier, "A generalized Jacobi theta functions and quasimodular forms," ibidem 165.

[19] P. Candelas, X. C. De La Ossa, P. S. Green and L. Parkes, "A pair of Calabi-Yau manifolds as an exactly soluble superconformal theory," Nucl. Phys. B 359, 21 (1991).

[20] S. Yamaguchi and S. T. Yau, "Topological string partition functions as polynomials," JHEP 0407, 047 (2004) arXiv:hep-th/0406078.

[21] M. Bershadsky, S. Cecotti, H. Ooguri and C. Vafa, "Holomorphic anomalies in topological field theories," Nucl. Phys. B 405, 279 (1993) arXiv:hep-th/9302103.

[22] M. Bershadsky, S. Cecotti, H. Ooguri and C. Vafa, "Kodaira-Spencer theory of gravity and exact results for quantum string amplitudes," Commun. Math. Phys. 165, 311 (1994) arXiv:hep-th/9309140.

[23] S. Hosono, A. Klemm, S. Theisen and S. T. Yau, "Mirror symmetry, mirror map and applications to complete intersection Calabi-Yau spaces," Nucl. Phys. B 433, 501 (1995) arXiv:hep-th/9406055.

[24] K. Hori, S. Katz, A. Klemm, R.Pandharipande, R. Thomas, C. Vafa, R. Vavil and E. Zaslow, Mirror Symmetry, AMS (2003).

[25] A. Ceresole, R. D'Auria, S. Ferrara and A. Van Proeyen, "Duality transformations in supersymmetric Yang-Mills theories coupled to supergravity," Nucl. Phys. B 444, 92 (1995) arXiv:hep-th/9502072.

[26] R. Minasian and G. W. Moore, "K-theory and Ramond-Ramond charge," JHEP 9711, 002 (1997) arXiv:hep-th/9710230].

[27] Y. K. Cheung and Z. Yin, "Anomalies, branes, and currents," Nucl. Phys. B 517, 69 (1998) arXiv:hep-th/9710206.

[28] I. Brunner, M. R. Douglas, A. E. Lawrence and C. Romelsberger, "D-branes on the quintic," JHEP 0008, 015 (2000) arXiv:hep-th/9906200.

[29] W. Fulton, "Intersection Theory," Springer (1998).

[30] V. I. Arnold, S. M. Gusein-Zade, A. N. Varchencko, "Singularities of diefferential maps I\& II," Birkhäuser, Basel (1985). 
[31] D. E. Diaconescu and C. Romelsberger, Nucl. Phys. B 574, 245 (2000) arXiv:hep-th/9910172.

[32] P. Mayr, "Phases of supersymmetric D-branes on Kaehler manifolds and the McKay correspondence," JHEP 0101, 018 (2001) arXiv:hep-th/0010223.

[33] S. Katz, A. Klemm and C. Vafa, "M-theory, topological strings and spinning black holes," Adv. Theor. Math. Phys. 3, 1445 (1999) [arXiv:hep-th/9910181].

[34] D. Ghoshal and C. Vafa, "C $=1$ String As The Topological Theory Of The Conifold," Nucl. Phys. B 453, 121 (1995) arXiv:hep-th/9506122].

[35] M. Marino and G. W. Moore, "Counting higher genus curves in a Calabi-Yau manifold," Nucl. Phys. B 543, 592 (1999) arXiv:hep-th/9808131.

[36] A. Klemm and M. Marino, "Counting BPS states on the Enriques Calabi-Yau," arXiv:hep-th/0512227.

[37] R. Gopakumar and C. Vafa, "M-theory and topological strings. I," arXiv:hep-th/9809187.

[38] R. Gopakumar and C. Vafa, "M-theory and topological strings. II," hep-th/9812127.

[39] C. Faber and R. Pandharipande, "Hodge Integrals and Gromov-Witten theory," arXiv:math.ag/9810173.

[40] T. Grimm, A. Klemm, M. Marino and M. Weiss, work in progress.

[41] C. Vafa, "A Stringy test of the fate of the conifold," Nucl. Phys. B 447, 252 (1995) arXiv:hep-th/9505023.

[42] E. Witten, "Two-Dimensional Gravity and intersection theory on moduli spaces," Surveys in Diff. Geometry 1 (1991) 243.

[43] A. Strominger, "Massless black holes and conifolds in string theory," Nucl. Phys. B 451, 96 (1995) arXiv:hep-th/9504090.

[44] R. Gopakumar and C. Vafa, "Branes and fundamental Groups," arXiv:hep-th/9712048].

[45] A. Klemm and P. Mayr, "Strong coupling singularities and non-Abelian gauge symmetries in $\mathrm{N}=2$ string theory," Nucl. Phys. B 469, 37 (1996) arXiv:hep-th/9601014. 
[46] A. Klemm and S. Theisen, "Considerations of one modulus Calabi-Yau compactifications: Picard-Fuchs equations, Kahler potentials and mirror maps," Nucl. Phys. B 389, 153 (1993) arXiv:hep-th/9205041.

[47] D. Maulik, N. Nekrasov, A. Okounkov and R. Pandharipande, Gromov-Witten theory and Donaldson-Thomas theory I, arXiv:math.AG/0312059].

[48] E. P. Verlinde, "Attractors and the holomorphic anomaly," arXiv:hep-th/0412139.

[49] M. Gunaydin, A. Neitzke and B. Pioline, "Topological wave functions and heat equations," arXiv:hep-th/0607200.

[50] Y.-H. Chen, Y. Yang, N. Yui, "Monodromy of Picard-Fuchs differential equations for Calabi-Yau threefolds," arXiv:math.AG/0605675].

[51] W. Chen and Y. Ruan, "Orbifold Gromow-Witten theory", Orbifolds in mathmatics and physics Madison WI 2001, Contemp. Math., vol 310, Amer. Math. Soc., Providence, RI, 2002 25-85.

[52] J. Bryan, T. Graber, R. Pandharipande, "The orbifold quantum cohomology of $C^{2} / Z_{3}$ and Hurwitz-Hodge integrals," arXiv:math.AG/0510335.

[53] T. Coates, A. Corti, H. Iritani and H.-H. Tseng, "Wall-Crossing in Toric Gromow-Witten Theory I: Crepant Examples", arXiv:math.AG/0611550.

[54] E. Witten, "Quantum background independence in string theory," Nucl. Phys. B 372, 187 (1992), arXiv:hep-th/9306122.

[55] A. B. Giventhal, "Symplectic geometry of Frobenius structures," Frobenius manifolds, Aspects. Math., E36, Vieweg, Wiesbaden, (91-112) 2004, T. Coates, "Giventhals Lagrangian Cone and $S^{1}$-Equivariant Gromow-Witten Theory," [arXiv:math.AG.0607808].

[56] C. Doran and J Morgan, "Mirror Symmetry and Integral Variations of Hodge Structure Underlying One Parameter Families of Calabi-Yau Threefolds," arXiv:math.AG/0505272.

[57] C. I. Lazaroiu, "Collapsing D-branes in one-parameter models and small/large radius duality," Nucl. Phys. B 605, 159 (2001) arXiv:hep-th/0002004.

[58] A. Klemm, M. Kreuzer, E. Riegler and E. Scheidegger, "Topological string amplitudes, complete intersection Calabi-Yau spaces and threshold corrections," JHEP 0505, 023 (2005) arXiv:hep-th/0410018. 
[59] W. Schmidt, "Variaton of Hodge structure: The singularities of the period mapping," Inventiones Math.22 (1973) 211-319.

[60] E. Cattani, A. Kaplan and W. Schmidt, "Degenerarions of Hodge Structures," Annals of Math. 123 (1986) 457-535.

[61] J. Harris, "Curves in Projective Space," Sem. de Math. Sup. Les Presses de l'University de Monteral (1982).

[62] http://uw.physics.wisc.edu/ strings/aklemm/highergenusdata/

[63] T. Bridgeland, "Derived categories of coherent sheaves," Survey article for ICM 2006, arXiv:math.AG/0602129].

[64] P. S. Aspinwall, "D-branes on Calabi-Yau manifolds," arXiv:hep-th/0403166.

[65] S. Katz, S. Strømme and J-M. Økland: "Schubert: A Maple package for intersection theory and enumerative geometry," http://www .uib.no/People/nmasr/schubert/.

[66] D. Gaiotto, A. Strominger and X. Yin, "The M5-brane elliptic genus: Modularity and BPS states," arXiv:hep-th/0607010.

[67] J. de Boer, M. C. N. Cheng, R. Dijkgraaf, J. Manschot and E. Verlinde, "A farey tail for attractor black holes," JHEP 0611, 024 (2006) arXiv:hep-th/0608059].

[68] F. Dennef and G. Moore, "From OSV to OSV," talk available at http://strings06.itp.ac.cn/ and work in progress.

[69] M. Rocek, C. Vafa and S. Vandoren, "Hypermultiplets and topological strings," JHEP 0602 (2006) 062 arXiv:hep-th/0512206. 